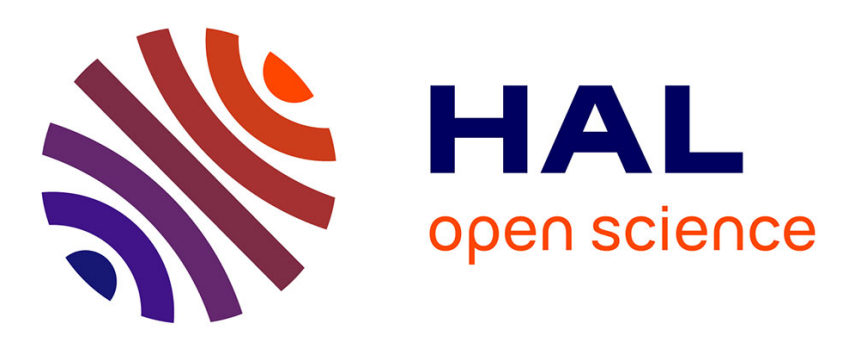

\title{
Unconventional semiclassical method for calculating the energetic values of diatomic molecules
}

\author{
Alexandru Popa
}

\section{To cite this version:}

Alexandru Popa. Unconventional semiclassical method for calculating the energetic values of diatomic molecules. Molecular Physics, 2011, 109 (04), pp.575-588. 10.1080/00268976.2010.539988 . hal00670737

\section{HAL Id: hal-00670737 \\ https://hal.science/hal-00670737}

Submitted on 16 Feb 2012

HAL is a multi-disciplinary open access archive for the deposit and dissemination of scientific research documents, whether they are published or not. The documents may come from teaching and research institutions in France or abroad, or from public or private research centers.
L'archive ouverte pluridisciplinaire HAL, est destinée au dépôt et à la diffusion de documents scientifiques de niveau recherche, publiés ou non, émanant des établissements d'enseignement et de recherche français ou étrangers, des laboratoires publics ou privés. 


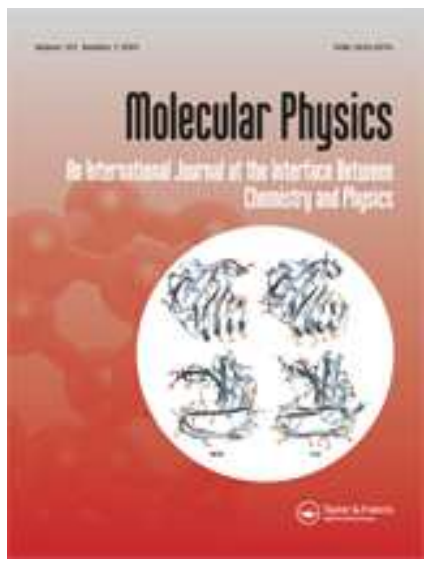

\section{Unconventional semiclassical method for calculating the energetic values of diatomic molecules}

\begin{tabular}{|r|l|}
\hline Journal: & Molecular Physics \\
\hline Manuscript ID: & TMPH-2010-0364.R1 \\
\hline $\begin{array}{r}\text { Manuscript Type: } \\
\text { Author: }\end{array}$ & Full Paper \\
\hline Keywords: & $\begin{array}{l}\text { diatomic molecules, energetic values, unconventional semiclassical } \\
\text { method }\end{array}$ \\
\hline & $\begin{array}{l}\text { PoPA, } \\
\text { Radiation Physics, Laser Department }\end{array}$ \\
\hline \multicolumn{2}{|c|}{ List of Authors: Laser, Plasma and } \\
\hline $\begin{array}{l}\text { Note: The following files were submitted by the author for peer review, but cannot be converted } \\
\text { to PDF. You must view these files (e.g. movies) online. }\end{array}$ \\
\hline $\begin{array}{l}\text { source files.zip } \\
\text { TMPH-2010-0364.Supplementary.Material.tex }\end{array}$ \\
\hline
\end{tabular}

\section{SCHOLARONE Manuscripts}


Molecular Physics

Vol. 00, No. 00, Month 2009, 1-55

\title{
FULL PAPER
}

\section{Unconventional semiclassical method for calculating the energetic values of diatomic molecules}

\author{
Alexandru Popa* \\ National Institute for Laser, Plasma and Radiation Physics, Laser Department, \\ P.O. Box MG-36, Bucharest, Romania 077125;
}

(Received 00 Month 200x; final version received 00 Month 200x)

\begin{abstract}
In previous papers we proved that the geometrical elements of the wave described by the Schrödinger equation, namely the wave surfaces and their normals, denoted by $C$ curves, are solutions of the Hamilton-Jacobi equations, written for the same system, in the case of stationary systems. The $C$ curves correspond to the same constants of motion as the eigenvalues of the Schrödinger equation. In two recent papers we presented a central field method for the calculation of the $C$ curves, and of the corresponding energetic values. The method was verified for the atoms $H e, L i, B e, B, C, N$ and $O$. In this paper we extend this method, using the symmetry properties of the systems, in the case of the diatomic molecules, with exemplification for $\mathrm{Li}_{2}, \mathrm{Be}_{2}, \mathrm{~B}_{2}, \mathrm{C}_{2}, \mathrm{LiH}, \mathrm{BeH}, \mathrm{BH}$ and $\mathrm{CH}$. The accuracy of the method is, as in the case of the atoms, comparable to the accuracy of the Hartree-Fock method, for the same system. This could be a potential useful result, because our approach predicts also basic properties of the molecules in discussion.
\end{abstract}

Keywords: diatomic molecules; energetic values; unconventional semiclassical method

\section{Introduction}

Over the last few years, a large number of semiclassical approaches were published in the field of atomic and molecular systems [1]-[5]. The semiclassical methods lead to solutions which are relatively easy to calculate, using the information obtained from the study of classical trajectories. In numerous papers the semiclassical eigenvalues of the bond states are related to the periodic trajectories [6]-[12], in others they are related to the phase manifolds generated by quasiperiodic trajectories [13]-[15]. In this family of topics we can consider also the study of the semiclassical trajectories with the aid of the propagator functions [16]-[20] or the application of a generalized semiclassical theory to multidimensional dynamical systems [21]. In this paper we present a multidimensional semiclassical analysis which applies to a wide class of atoms and molecules.

In a series of papers [22]-[24] we presented a method for calculating the energetic values of atomic and molecular systems, which is based on the following property [22]: the energy of the Schrödinger equation can be rigorously calculated by line integrals of analytical functions, if the Hamilton-Jacobi equation, written for the same system, is satisfied by a periodical trajectory. We proved [24] that this property is a consequence of the fact that, for stationary systems, the Schrödinger

*Email: ampopa@rdslink.ro 
equation is equivalent to the wave equation. Starting from this equivalence, we have proposed [24]-[26] a wave model for stationary atomic and molecular systems. In recent papers $[27,28]$ we have elaborated a central field method for calculation of energetic values of atomic systems, which is based on the above wave model. The central point of this method is the the following property $[25,26]$ : the geometrical elements of the wave described by the Schrödinger equation, namely the wave surfaces and their normals (the $C$ curves) are rigorously solutions of the HamiltonJacobi equation, written for the same system. We have verified this method for a large number of atoms, and we found that its accuracy is comparable to the accuracy of the Hartree-Fock method applied to the same atoms.

In this paper we use symmetry properties of the systems to show that the central field method for calculating the energetic values $[27,28]$ can be extended to diatomic molecules, both homonuclear and heteronuclear, with the same accuracy as that of the Hartree-Fock method. We compare our data with theoretical data taken from The Computational Chemistry Comparison and Benchmark Database (CCCBDB) [29]. In addition, the method presented in this paper predicts basic properties of the molecules [30,31], such as the existence of the simple, double and triple bonds in the case of the $C-C$ bond, the symmetry properties of these bonds, the fact that in the case of the $C_{2}$ molecule the bond is double, and it also explains the ionic character of the $\mathrm{LiH}$ bond.

The paper is structured as follows. In Section 2 we present briefly the relations and results from previous papers which are necessary in this paper. In Section 3 we give exact solutions to the equations of the $C$ curves, in the case of simple, double and triple covalent bonds of homonuclear molecules and in the case of ionic and covalent bonds of heteronuclear molecules. In Section 4 we present the method for calculating the energetic values, and in Section 5 we apply the method to several molecules. We present the computation details in two typical cases, $L i_{2}$ and $B e_{2}$, and we give the results of the computation in the other cases investigated: the $B_{2}$, $\mathrm{C}_{2}, \mathrm{LiH}, \mathrm{BeH}, \mathrm{BH}$ and $\mathrm{CH}$. Details of the computation for the these molecules are given in the Supplementary Online Material for this article, where we also give the Mathematica 7 scripts containing all the calculations from this paper.

Throughout the paper the equations are written in the International System.

\section{Preliminary results}

In this section we present the relations which have been deduced in previous papers [25]-[28] and which are necessary in this paper. In Refs. [25, 26] we studied the wave properties of a discrete system composed of $N$ electrons and $N^{\prime}$ nuclei. The Cartesian coordinates of the electrons are $x_{a}, y_{a}, z_{a}$, where $a$ takes values between 1 and $N$. Our analysis is made in the space $R^{3 N}$ of the electron coordinates, which are denoted by $q_{j}$ (where $\left.q_{1}=x_{1}, q_{2}=y_{1}, q_{3}=z_{1}, \ldots, q_{3 N}=z_{N}\right), j$ taking values between 1 and $3 N$. We denote by $q=\left(q_{1}, q_{2}, \ldots, q_{3 N}\right)$ the coordinates of a point in the space $R^{3 N}$.

We consider a system which fulfills the following initial hypotheses: (h1) The system is closed and conservative (i.e. the total energy, denoted by $E$, is constant and the potential energy, denoted by $U$, does not depend explicitly on time); (h2) The total energy has real negative values (i.e. the system is in a bound state); (h3) The behavior of the system is completely described by the Schrödinger equation; (h4) The relativistic and magnetic effects are neglected; (h5) The nuclei motion is neglected. We proved $[25,26]$ that the following properties are valid for this system:

(p1) In the case of the stationary systems, the Schrödinger and wave equations are rigorously equivalent, and the characteristic surface of the wave equation (which has 


\section{Molecular Physics}

Molecular Physics

the significance of a wave surface), denoted by $\Sigma$, and its normals, denoted by $C$, are solutions of the Hamilton-Jacobi, written for the same system, and corresponding to constants of motion identical to the eigenvalues of the Schrödinger equation.

(p2) The motion of the surface $\Sigma$ is periodical, and the $C$ curves are closed (see Section 4 of Ref. [25]).

(p3) The generalized Bohr quantization condition

$$
\Delta_{C} S_{0}=n h
$$

is valid for the $C$ curve (see Section 5 of Ref. [26]). Here $S_{0}$ is the reduced action function, $\Delta_{C} S_{0}$ is the variation of the function $S_{0}$ which corresponds to the curve $C$ and $n$ is the principal quantum number.

In the case of systems for which the separation of variables is possible, namely when the function $S_{0}$ can be written $S_{0}=\sum_{a} S_{0 a}$, where $S_{0 a}=S_{0 a}\left(x_{a}, y_{a}, z_{a}\right)$, the following quantization relation is valid [26]

$$
\Delta_{C_{a}} S_{0 a}=n_{a} h
$$

where $n_{a}$ is the principal quantum number associated to the motion of the electron $a$ and $C_{a}$ is the curve corresponding to the electron $a$.

The property (p1) establishes a direct connection between the Schrödinger and Hamilton-Jacobi equations in the case of the stationary systems. A similar connection, which has been derived through entirely different method, is presented in [32], where it is shown that the discontinuities of the partial second derivatives of the wave function propagate following the trajectories determined by the Hamilton-Jacobi equation, written for the same system.

The particular curve of a given electron is obtained from the projection of the trajectory $C$ from the $R^{3 N}$ space of coordinates to the three dimensional space of that electron. For example, the curve of electron $a$ is obtained from the projection of the curve $C$ to the space of coordinates $x_{a}, y_{a}, z_{a}$. The projection is also a closed curve, denoted by $C_{a}^{\prime}$, where $a=1,2, \ldots, N$. When the separation of variables is possible, we have $C_{a}^{\prime} \equiv C_{a}$.

The above properties imply that the problem of calculating the energetic values of atomic and molecular systems reduces to the calculation of the $C_{a}^{\prime}$ or $C_{a}$ curves, or, in other words, to the calculation of the systems' periodic solutions. In previous papers $[27,28]$ we have shown that the problem can be solved exactly, or with good accuracy, by a central field method, which makes possible the separation of the variables, and, consequently, the calculation of the $C_{a}$ curves. In this case we have applied the quantization condition, namely (2) for each $C_{a}$ curve and have obtained the following relations for the calculation of the total energy, which are 
valid for all the atoms which have been analyzed in Refs. [27, 28]:

$$
\begin{gathered}
E=E_{1}+E_{2}+\sum_{a \geq 3} E_{a} \\
E_{1}=E_{2}=-\frac{Z_{1} K_{1}}{r_{1}}+\frac{m}{2}\left(\frac{d r_{1}}{d t}\right)^{2}=-\frac{R_{\infty} Z_{1}^{2}}{n_{1}^{2}} \\
E_{a}=-\frac{Z_{a} K_{1}}{r_{a}}+\frac{m}{2}\left(\frac{d r_{a}}{d t}\right)^{2}=-\frac{R_{\infty} Z_{a}^{2}}{n_{a}^{2}} \\
Z_{1}=Z_{2}=Z-s_{12 e}-\sum_{a \geq 3} s_{1 a e} \text { with } s_{12 e}=\frac{1}{4} \\
Z_{a}=Z-2 s_{a 1 e}-\sum_{b \geq 3 ; b \neq a} s_{a b e}
\end{gathered}
$$

Here $r_{a}$ is the norm of position vector of the electron $e_{a}$ in a Cartesian system of coordinates with origin at the nucleus, $m$ is the electron mass, $t$ is the time, $Z$ is the order number of the nucleus, and $K_{1}$ is a constant given by the relation

$$
K_{1}=\frac{e^{2}}{4 \pi \varepsilon_{0}}
$$

where $e$ and $\varepsilon_{0}$ are the absolute value of the electron charge and the vacuum permitivity, respectively. In these equations $E_{1}$ and $E_{2}$ are the energies of the $1 \mathrm{~s}$ electrons, $E_{a}$ is the energy of the valence electron $a$, where $a \geq 3$ for $Z \geq 3, Z_{1}$ and $Z_{2}$ are the effective order numbers of nucleus, which enter in the motion equations of the $1 s$ electrons, $Z_{a}$ is the effective order number of nucleus which enters in the equation of electron $a$ and $R_{\infty}$ is the Rydberg energy. The quantities $s_{1 a e}$ and $s_{a 1 e}$ are the effective reciprocal screening coefficients between the $1 s$ electrons and the valence electrons $e_{a}$, which are calculated with the aid of the equation system (59)-(62) from Ref. [27], when eccentricity of the curve $C_{a}$ is very closed to unit, and with the aid of the system (73)-(77) from Ref. [27], when $C_{a}$ is an elliptic curve. The quantities $s_{12 e}$ and $s_{a b e}$ are, respectively, the effective reciprocal screening coefficients between the $1 s$ electrons and between the valence electrons, which are calculated with the aid of the relation (7) from Ref. [28], which is:

$$
s_{a b e}=s_{b a e}=\frac{1}{4 \sin \left(\alpha_{a b} / 2\right)}
$$

where $\alpha_{a b}$ is the angle between the two straight lines which pass through nucleus and the average positions of the two electrons.

The interaction energy between two electrons, let us say $e_{a}$ and $e_{b}$, can be written: $[27,28]$

$$
U_{a b e}=\frac{K_{1}}{\left|\bar{r}_{a}-\bar{r}_{b}\right|}=\frac{K_{1} s_{a b e}}{r_{a}}+\frac{K_{1} s_{b a e}}{r_{b}}
$$

From this relation and from (4)-(7), it follows that part of the interaction energy enters in the expression of $E_{a}$, by the term $K_{1} s_{a b e} / r_{a}$, while the other part, $K_{1} s_{b a e} / r_{b}$, enters in the expression of $E_{b}$.

The expression of the total energy, given by (3), contains, in adition, a correction energy due to the spin magnetic interaction of the $1 s$ electrons, which has been 


\section{Molecular Physics}

Molecular Physics

deduced in Ref. [27]. This term is

$$
E_{m 1 s}=R_{\infty} \frac{Z_{1}^{\frac{3}{2}}}{8 n_{1}^{3}} \quad \text { with } n_{1}=1
$$

The model represented by equations (3)-(10) has two properties which are both attributes of a central field type solution. The first is that the motion of an arbitrary electron is similar to that in a hydrogenoid system, and it takes place in the averaged field of the other electrons. The effect of the interaction with these electrons is included in the effective order number of the nucleus. The second property, which is useful in applications, is that adding a supplementary electron to the system leads only to the modification of the effective order numbers corresponding to the other electrons, while their equations remain unchanged.

On the other hand, in Ref. [33] it is shown that, for a multielectron system, the energetically most favorable geometric configuration minimizes the electronelectron repulsion and, generally, this configuration corresponds to the maximum symmetry of the geometrical configuration of the electrons. Our results [27, 28] confirm this property, because the $C_{a}$ curves, as result from our treatment, have also a configuration with maximum symmetry. For example, the $C_{a}$ curves of the valence electrons in the case of helium and beryllium are two symmetrical ellipses, having eccentricities very close to unity, for boron the $C_{a}$ curves are three symmetrical ellipses, whose axes are oriented toward the corners of an equilateral triangle, and so on. A strong simplification of the calculation of the total energy results if we apply this property. In this case we suppose the average positions of the electrons, which have maximum symmetry, calculate the $s_{1 a e}, s_{a 1 e}$ and $s_{a b e}$ and the total energy results directly from the equations (4) and (5). The average distance and the maximum distance of the electron from the nucleus, denoted, respectively, by $\tilde{r}_{a}$ and $r_{M}$, are [28]

$$
\tilde{r}_{a}=\frac{n_{a}^{2} a_{0}}{Z_{a}}\left(1+\frac{e^{2}}{2}\right) \text { and } r_{M}=\frac{n_{a}^{2} a_{0}}{Z_{a}}(1+e)
$$

where $a_{0}$ is the Bohr radius and $e$ is the eccentricity of the $C_{a}$ curve.

We have applied this method and resulted that the accuracy of the calculation of the energy is comparable with the accuracy of the Hartree-Fock method for the atoms $\mathrm{He}, \mathrm{Li}, \mathrm{Be}, \mathrm{B}, \mathrm{C}, \mathrm{N}$ and $O$ (see Table 1 from Ref. [27] and Table 4 from Ref. [28]). We will use this method in the case of molecules, for the calculation of the energies of the electrons which do not participate directly to the bonds.

\section{Calculation of the $C_{a}$ curves corresponding to simple, double and triple bonds of homonuclear molecules and to ionic and covalent bonds of heteronuclear molecules}

In this section we present calculations of the $C_{a}$ curves corresponding to the bond electrons in homonuclear and heteronuclear molecules. The $C_{a}$ curves for the simple bond of homonuclear molecules were presented in Refs. [23, 24]. In this paper we present them, taking into account the new data resulted from the central field method, applied in the case of atoms. The new topics presented in this paper refers to the double and triple bonds of the homonuclear molecules, to the modeling of the heteronuclear molecules and to the explaining of a lot of properties of the molecules, which result from our calculations. 


\section{Notations.}

The symbols used in the equations that describe the behavior of the molecules contain supplementary indexes, which refer to different nuclei of the molecule. These indexes are, as follows.

1) The $1 s$ electrons which move in the field of the nucleus $n_{A}$ are denoted by $e_{A 1}, e_{A 2}$, while the valence electrons which move in the field of the nucleus $n_{A}$ and do not participate to the bond are denoted by $e_{A 3}, e_{A 4}, e_{A 5}$, and so on. Similar notations correspond to electrons which move in the field of the nucleus $n_{B}$. The bond electrons are denoted by $e_{1}, e_{2}, e_{3}$, and so on.

2) In the case of the homonuclear diatomic molecules, the nuclei order numbers, denoted by $Z_{A}$ and $Z_{B}$, are equal. In this case, the order numbers corresponding to the electrons $e_{A i}$ and $e_{j}$, where $i, j=1,2, \ldots$, are denoted, respectively, by $Z_{A i}$ and $Z_{j}$. The screening coefficients between electrons $e_{A i}$ and $e_{A k}$ are denoted by $s_{A i, A k}$ and $s_{A k, A i}$, those between the bond electrons $e_{j}$ and $e_{k}$ are denoted by $s_{j, k}$ and $s_{k, j}$, and the screening coefficients between the electrons $e_{A i}$ and $e_{j}$ are denoted by $s_{A i, j}$ and $s_{j, A i}$.

3) The effective order number of the nucleus $n_{A}$ in interactions between nuclei includes the effect of the $1 s$ electrons and the effect of the electrons that do not participate to the bond. In this case the effective order number of $n_{A}$ is denoted by $Z_{n A}$.

\section{1. $C_{a}$ curves for simple, double and triple bonds of homonuclear molecules}

We consider a molecule composed of two fixed identical nuclei, denoted by $n_{A}$ and $n_{B}$, two $1 s$ electrons in the vicinity of the $n_{A}$ nucleus, denoted by $e_{A 1}$ and $e_{A 2}$, two $1 s$ electrons in the vicinity of the $n_{B}$ nucleus, denoted by $e_{B 1}$ and $e_{B 2}$, and $N_{b}$ valence electrons which participate to the bond, denoted by $e_{1}, e_{2}, \ldots, e_{N_{b}}$. The Cartesian coordinates of the nuclei are $n_{A}(-\sigma, 0,0)$ and $n_{B}(\sigma, 0,0)$ and their order number is equal to $Z_{A}$. The equations of motion for the electrons are as follows:

$$
\begin{array}{r}
-\frac{K_{1} Z^{\prime}\left(\bar{r}_{a}-\sigma \bar{i}\right)}{\left|\bar{r}_{a}-\sigma \bar{i}\right|^{3}}-\frac{K_{1} Z^{\prime}\left(\bar{r}_{a}+\sigma \bar{i}\right)}{\left|\bar{r}_{a}+\sigma \bar{i}\right|^{3}}+\sum_{b} \frac{K_{1}\left(\bar{r}_{a}-\bar{r}_{b}\right)}{\left|\bar{r}_{a}-\bar{r}_{b}\right|^{3}}=m \frac{d^{2} \bar{r}_{a}}{d t^{2}} \\
\text { where } a, b=1,2, \ldots N_{b} \text { and } a \neq b
\end{array}
$$

Here $\bar{r}_{a}$ are the position vectors of the $e_{a}$ electrons, which have their origin in the origin of the Cartesian coordinate axes, whose versors are denoted by $\bar{i}, \bar{j}, \bar{k}$, and $Z^{\prime}$ is an order number which includes the effect of the $1 s$ electrons. It is given by the relation $Z^{\prime}=Z_{A}-2 s_{a, A 1}$.

This system has three exact solutions similar to the solutions of the valence bond electrons. More precisely, there is one solution for each one of the following three cases: a) for $N_{b}=2$, which corresponds to simple bond; b) for $N_{b}=4$, which corresponds to the double bond and c) for $N_{b}=6$, which corresponds to the triple bond.

\subsubsection{Simple bond $\left(N_{b}=2\right)$}

The solution of the system in this case is presented in Section 3 of Ref. [23]. This solution is:

$$
\bar{r}_{1}=-\bar{r}_{2}=\bar{r} \text { for } \bar{r} \cdot \bar{k}=0
$$

It is easy to show that, in virtue of (14), the solution the system represented by 
(13) reduces to the solution of the following equation

$$
-\frac{K_{1} Z^{\prime}\left(\bar{r}_{a}-\sigma \bar{i}\right)}{\left|\bar{r}_{a}-\sigma \bar{i}\right|^{3}}-\frac{K_{1} Z^{\prime}\left(\bar{r}_{a}+\sigma \bar{i}\right)}{\left|\bar{r}_{a}+\sigma \bar{i}\right|^{3}}+\frac{K_{1} \bar{r}_{a}}{4\left|\bar{r}_{a}\right|^{3}}=m \frac{d^{2} \bar{r}_{a}}{d t^{2}}
$$

which is valid for $a=1$ or $a=2$. This solution corresponds to symmetrical velocities. The symmetry of the velocities results from the total derivative of (14) with respect to time, which gives $\bar{v}_{1}=-\bar{v}_{2}=\bar{v}$. In virtue of (14), (15) is equivalent to the following two scalar equations, in the plane $(x, y)$ :

$$
\begin{aligned}
& -\frac{K_{1} Z^{\prime}(x-\sigma)}{\left[(x-\sigma)^{2}+y^{2}\right]^{3 / 2}}-\frac{K_{1} Z^{\prime}(x+\sigma)}{\left[(x+\sigma)^{2}+y^{2}\right]^{3 / 2}}+\frac{1}{4} \frac{K_{1} x}{\left(x^{2}+y^{2}\right)^{3 / 2}}=m \frac{d^{2} x}{d t^{2}} \\
& -\frac{K_{1} Z^{\prime} y}{\left[(x-\sigma)^{2}+y^{2}\right]^{3 / 2}}-\frac{K_{1} Z^{\prime} y}{\left[(x+\sigma)^{2}+y^{2}\right]^{3 / 2}}+\frac{1}{4} \frac{K_{1} y}{\left(x^{2}+y^{2}\right)^{3 / 2}}=m \frac{d^{2} y}{d t^{2}}
\end{aligned}
$$

The numerical solution of the above system of equations is given in Ref. [23], and it leads to the curve $C_{1}$ that corresponds to electron $e_{1}$. The curve $C_{2}$, corresponding to electron $e_{2}$, is symmetrical to the curve $C_{1}$.

The atomic curves for the fundamental states of atoms, calculated in Refs. [27] and [28], are ellipses with eccentricities very close to unity. For this reason, in this paper we restrict ourselves to the case in which the molecular $C_{1}$ curve is composed of two atomic curves, denoted by $C_{1 A}$ and $C_{1 B}$, which are ellipses with eccentricities very close to unity, as shown in Fig. 1. At the scale of the figure, these ellipses are drawn as straight lines in the vicinities of the nuclei, in spite of the fact that the curve $C_{1 A}$ surrounds the nucleus $n_{A}$, while the curve $C_{1 B}$ surrounds the nucleus $n_{B}$. The curves $C_{1}$ and $C_{2}$ depend on three parameters: $E_{b}, \sigma$ and $a$, where $E_{b}$ is the total energy of the particles that participate to the bond, and $a$ is the maximum distance between the electron and the line on which the two nuclei are situated [23]. In Fig. 1 the electrons are situated in average positions, in agreement with (12). 


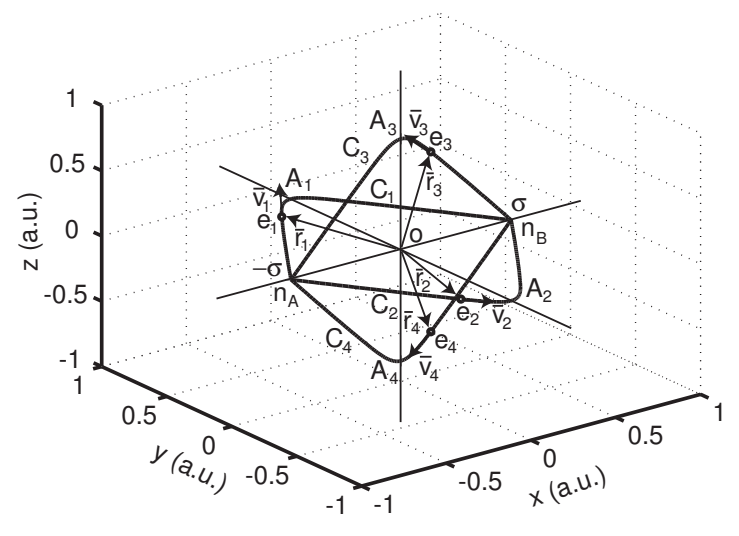

(a)

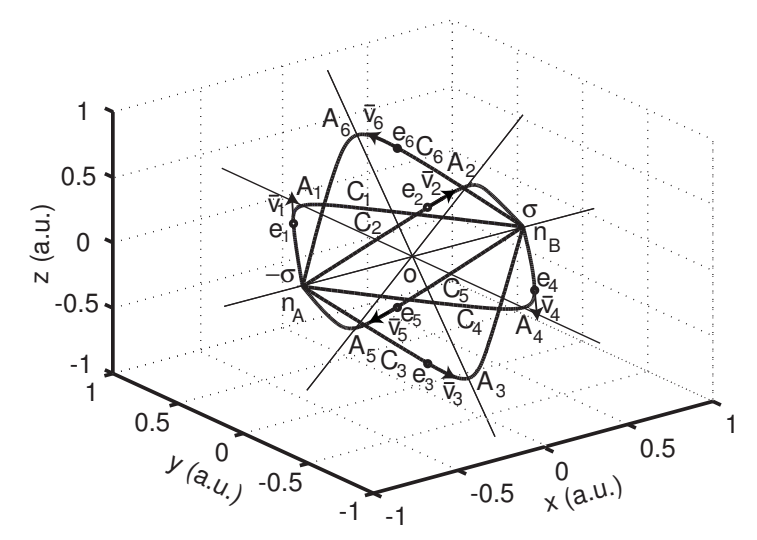

(b)

Figure 2. $C_{a}$ curves for (a) double bond and (b) triple bond in homonuclear diatomic molecules.

\subsubsection{Double bond $\left(N_{b}=4\right)$}

In this case the solution of the system represented by (13) is very similar to the solution for the simple bond. This solution is:

$$
\begin{gathered}
\bar{r}_{1}=-\alpha \bar{i}+\beta \bar{j} \\
\bar{r}_{2}=-\alpha \bar{i}-\beta \bar{j} \\
\bar{r}_{3}=\alpha \bar{i}+\beta \bar{k} \\
\bar{r}_{4}=\alpha \bar{i}-\beta \bar{k}
\end{gathered}
$$

where $\alpha$ and $\beta$ are real variables. It is easy to show that, in virtue of (18)-(21), the solution of the system represented by (13) reduces to the solution of the following equation, in which the unknown is $\bar{r}_{a}$

$$
\begin{gathered}
-\frac{K_{1} Z^{\prime}\left(\bar{r}_{a}-\sigma \bar{i}\right)}{\left|\bar{r}_{a}-\sigma \bar{i}\right|^{3}}-\frac{K_{1} Z^{\prime}\left(\bar{r}_{a}+\sigma \bar{i}\right)}{\left|\bar{r}_{a}+\sigma \bar{i}\right|^{3}}+\frac{\bar{r}_{a}-\left(\bar{r}_{a} \cdot \bar{i}\right) \bar{i}}{4\left[\bar{r}_{a}^{2}-\left(\bar{r}_{a} \cdot \bar{i}\right)^{2}\right]^{3 / 2}}+ \\
\frac{\bar{r}_{a}+\left(\bar{r}_{a} \cdot \bar{i}\right) \bar{i}}{\sqrt{2}\left[\bar{r}_{a}^{2}+\left(\bar{r}_{a} \cdot \bar{i}\right)^{2}\right]^{3 / 2}}=m \frac{d^{2} \bar{r}_{a}}{d t^{2}}
\end{gathered}
$$

This equation is valid for any bond electron. For example, Eq. (22) written for $\bar{r}_{1}$ (i.e. $a=1$ ) is obtained from (13) in which we plug in the solutions of $\bar{r}_{2}, \bar{r}_{3}$ and $\bar{r}_{4}$ given by (19)-(21). Same procedure can be used to derive (22) for $a=2,3,4$. The solution to (22) corresponds to symmetrical velocities. The symmetry of the velocities results from the total derivative of (18)-(21) with respect to time.

Equation (22) leads again to a plane $C_{1}$ curve, for the electron $e_{1}$. It is equivalent to two scalar equations, very similar to $(16,17)$. An identical numerical solution, 


\section{Molecular Physics}

Molecular Physics

as that shown in Ref. [23], leads again to a molecular curve, which is composed of two elliptic quasilinear curves, when the minimum distance between electron and the nucleus is negligible. The curves for the other electrons are symmetrical, in agreement to (18)-(21). These curves, which result from (22), are represented in Fig. 2(a). From this figure we see that, in the vicinities of the nuclei, $C_{1}$ and $C_{2}$ are two symmetrical curves, in the plane $(x, y)$, similar to the valence curves in the case of the helium atom, or in the case of beryllium. The other curves, $C_{3}$ and $C_{4}$ are two symmetrical curves, with the same properties, situated in the plane $(x, z)$.

\subsubsection{Triple bond $\left(N_{b}=6\right)$}

In this case the symmetrical solution of the system represented by (13) is as follows:

$$
\begin{gathered}
\bar{r}_{1}=-\alpha \bar{i}+\beta \bar{j} \\
\bar{r}_{2}=-\alpha \bar{i}-\frac{1}{2} \beta \bar{j}+\frac{\sqrt{3}}{2} \beta \bar{k} \\
\bar{r}_{3}=-\alpha \bar{i}-\frac{1}{2} \beta \bar{j}-\frac{\sqrt{3}}{2} \beta \bar{k} \\
\bar{r}_{4}=\alpha \bar{i}-\beta \bar{j} \\
\bar{r}_{5}=\alpha \bar{i}+\frac{1}{2} \beta \bar{j}-\frac{\sqrt{3}}{2} \beta \bar{k} \\
\bar{r}_{6}=\alpha \bar{i}+\frac{1}{2} \beta \bar{j}+\frac{\sqrt{3}}{2} \beta \bar{k}
\end{gathered}
$$

where $\alpha$ and $\beta$ are real variables. It is easy to show that, as in the previous case, in virtue of (23)-(28), the solution the system represented by (13) reduces to the solution of the following equation in which the unknown is $\bar{r}_{a}$

$$
\begin{aligned}
-\frac{K_{1} Z^{\prime}\left(\bar{r}_{a}-\sigma \bar{i}\right)}{\left|\bar{r}_{a}-\sigma \bar{i}\right|^{3}}-\frac{K_{1} Z^{\prime}\left(\bar{r}_{a}+\sigma \bar{i}\right)}{\left|\bar{r}_{a}+\sigma \bar{i}\right|^{3}}+ & \frac{\bar{r}_{a}}{4\left|r_{a}\right|^{3}}+\frac{\bar{r}_{a}+3\left(\bar{r}_{a} \cdot \bar{i}\right) \bar{i}}{\left[\bar{r}_{a}^{2}+3\left(\bar{r}_{a} \cdot \bar{i}\right)^{2}\right]^{3 / 2}}+ \\
& \frac{\bar{r}_{a}-\left(\bar{r}_{a} \cdot \bar{i}\right) \bar{i}}{\sqrt{3}\left[\bar{r}_{a}^{2}-\left(\bar{r}_{a} \cdot \bar{i}\right)^{2}\right]^{3 / 2}}=m \frac{d^{2} \bar{r}_{a}}{d t^{2}}
\end{aligned}
$$

This equation is valid for any value of $a$. For example we obtain (29) for $a=1$ by introducing the solutions for $\bar{r}_{2}, \bar{r}_{3}, \ldots, \bar{r}_{6}$, given by (24)-(28), in (13) written for the electron $e_{1}$. In the same manner we obtain the above equation for $a=2, \ldots 6$. The solution to (29) corresponds again to symmetrical velocities.

Equation (29) leads to a plane $C_{1}$ curve for electron $e_{1}$. It is equivalent, as in the previous case, to two scalar equations, very similar to (16), (17). An identical numerical solution, as that shown in Ref. [23], leads again to a molecular curve, which is composed of two elliptic quasilinear curves. The curves for the other electrons are symmetrical, in agreement to (23)-(28). These curves, which result from (29), are represented in Fig. 2(b). From this figure we see that, in the vicinities of the nuclei, $C_{1}, C_{2}, C_{3}$ are three symmetrical curves, similar to the curves of the valence electrons, in the case of the boron atom. The other curves, $C_{4}, C_{5}$ and $C_{6}$ are three symmetrical curves, with the same properties. 


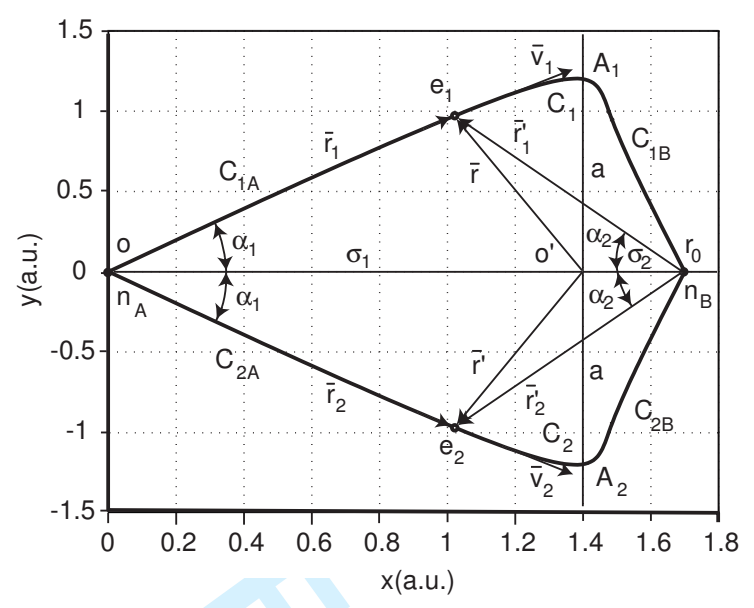

(a)

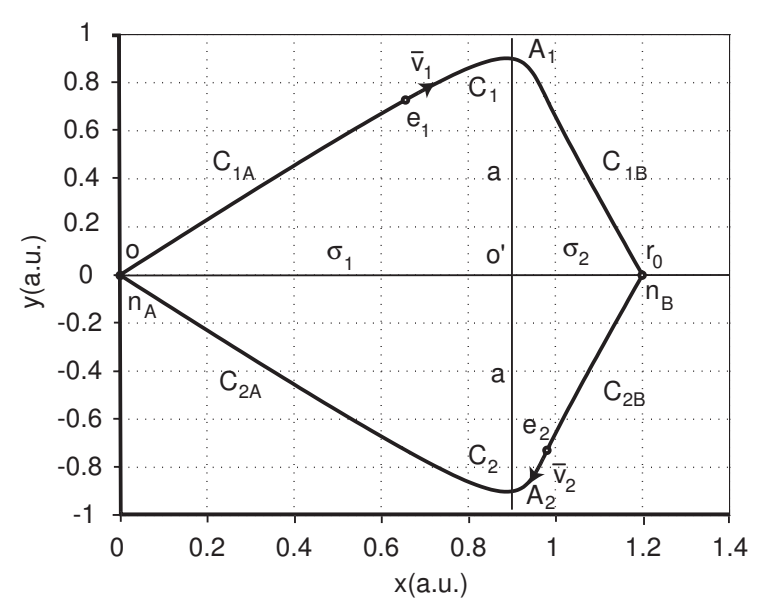

(b)

Figure 3. $C_{a}$ curves for (a) ionic bond and (b) covalent bond in heteronuclear diatomic molecules.

\section{2. $C_{a}$ curves for ionic and covalent bonds in heteronuclear molecules}

We consider a heteronuclear molecule composed of two fixed different nuclei, denoted by $n_{A}$ and $n_{B}$, two $1 s$ electrons in the vicinity of the $n_{A}$ nucleus, denoted by $e_{A 1}$ and $e_{A 2}$, and two valence electrons which participate to the bond, denoted by $e_{1}$ and $e_{2}$, as shown in Fig. 3(a). The Cartesian coordinates of the nuclei are $n_{A}(0,0,0)$ and $n_{B}\left(r_{0}, 0,0\right)$ and their order numbers are, respectvely, equal to $Z_{A}$ and $Z_{B}$, where $Z_{A}>A_{B}$. The equations of motion for electrons are as follows:

$$
\begin{array}{r}
-\frac{K_{1} Z_{A}^{\prime} \bar{r}_{a}}{\left|\bar{r}_{a}\right|^{3}}-\frac{K_{1} Z_{B}\left(\bar{r}_{a}-r_{0} \bar{i}\right)}{\left|\bar{r}_{a}-r_{0} \bar{i}\right|^{3}}+\sum_{b} \frac{K_{1}\left(\bar{r}_{a}-\bar{r}_{b}\right)}{\left|\bar{r}_{a}-\bar{r}_{b}\right|^{3}}=m \frac{d^{2} \bar{r}_{a}}{d t^{2}} \\
\text { where } a, b=1,2 \text { and } a \neq b
\end{array}
$$

Here $\bar{r}_{a}$ are the position vectors of the electrons having the origin in the origin of the Cartesian coordinate axes, and $Z_{A}^{\prime}$ is an effective order number which includes the effect of the $1 s$ electrons.

This system has the following symmetrical solution:

$$
x_{1}=x_{2}, \quad y_{1}=-y_{2} \text { and } z_{1}=z_{2}=0
$$

The symmetry of the velocities results from the total derivative of these relations with respect to time.

An identical procedure, as that presented in the previous subsection, shows that, for this solution, the above system reduces to the following two scalar equations in the plane $(x, y)$ :

$$
\begin{gathered}
-\frac{K_{1} Z_{A}^{\prime} x_{a}}{\left(x_{a}^{2}+y_{a}^{2}\right)^{3 / 2}}-\frac{K_{1} Z_{B}\left(x_{a}-r_{0}\right)}{\left[\left(x_{a}-r_{0}\right)^{2}+y_{a}^{2}\right]^{3 / 2}}=m \frac{d^{2} x_{a}}{d t^{2}} \\
-\frac{K_{1} Z_{A}^{\prime} y_{a}}{\left(x_{a}^{2}+y_{a}^{2}\right)^{3 / 2}}-\frac{K_{1} Z_{B} y_{a}}{\left[\left(x_{a}-r_{0}\right)^{2}+y_{a}^{2}\right]^{3 / 2}}+\frac{K_{1} y_{a}}{4\left|y_{a}\right|^{3}}=m \frac{d^{2} y_{a}}{d t^{2}}
\end{gathered}
$$

which are valid for $a=1,2$.

The numerical solution of the above system of equations is almost identical to 
the solution of the system represented by (16), (17), which is given in Ref. [23]. Same numerical method presented in Ref. [23] can be used to compute curves $C_{1}$ and $C_{2}$ corresponding to the electrons $e_{1}$ and $e_{2}$, which are shown in Fig. 3(a). In this figure, the coordinates of the points $A_{1}$ and $A_{2}$ are, respecively, $\left(\sigma_{1}, a, 0\right)$ and $\left(\sigma_{1},-a, 0\right)$. We have also $\sigma_{2}=r_{0}-\sigma_{1}$. The difference between the curve $C_{1}$ resulted from (16), (17) and the curve $C_{1}$ resulted from (32), (33) is that the latter does not have an axis of symmetry parallel to the oy axis, as illustrated by the comparison between Figs. 1 and 3(a), respectively. On the other hand the curves $C_{1}$ and $C_{2}$ shown in Fig. 3(a) are symmetric to each other with respect to the ox axis. Each of these curves is composed of two quasilinear ellipses. The analysis of this figure shows the existence of two phases, the phase $A$, when the electrons are situated on the curves $C_{1 A}$ and $C_{2 A}$, in the vicinity of the nucleus $n_{A}$, and the phase $B$, when the electrons are situated on the curves $C_{1 B}$ and $C_{2 B}$, in the vicinity of the nucleus $n_{B}$. These curves correspond to the ionic bond because, due to the nonsymmetry, in the majority of the time, the electrons are in the vicinity of the nucleus whose order number is smaller.

A similar analysis, as that presented above, leads to the $C_{a}$ curves corresponding to the covalent bond in heteronuclear diatomic molecule. In this case the $e_{1}$ and $e_{2}$ electrons are situated in the vicinities of different nuclei, as shown in Fig. 3(b). Unlike the ionic bond, in the covalent bond the electrons move alternatively in the fields of different nuclei, and their charge is disposed in the vicinities of both nuclei.

In this section we have calculated the $C_{a}$ curves when the bond electrons move in the fields of two nuclei and the system contains only valence electrons which participate to the bond. In Appendix we proved that the motion of a bond electron in the case of homonuclear molecules with simple bond is similar to that in a central field. An example of such an electron is $e_{1}$ which moves on curves $C_{1 A}$ and $C_{1 B}$. This property results from relation (A3). Identical properties are proved in the Supplementary Online Material for other types of bonds. It follows from Section 2 that, in virtue of the second property of motion in the central field, adding a supplementary electron that does not participate to the bond in the field of the nucleus $n_{A}$ does not change the equation of motion of the electron $e_{1}$. The only change is the fact that the expression of $Z_{1}$ contains a supplementary screening coefficient corresponding to the interaction between $e_{1}$ and the supplementary electron. In the next section we will see that for the general case of the molecules which contains electrons which do not participate to the bond, the relations from Appendix remain valid, with the only difference that the expressions of the order numbers are changed.

\section{Analytical method for the calculation of energetic values of diatomic molecules}

In this section, we present a general analytical method for the calculation of the energetic values and geometric parameters of diatomic molecules, which leads to values in close agreement with the experimental values for all the molecules analyzed in this paper.

The structure of the molecule is illustrated by Fig. 4. Despite the fact that in this figure we consider the case of a homonuclear molecule with a simple bond, the method presented in this section is valid for all types of bonds. The molecule shown in Fig. 4 is composed of two nuclei denoted by $n_{A}$ and $n_{B}$ and having $Z_{A}=Z_{B}$, four $1 s$ electrons, denoted by $e_{A 1}, e_{A 2}, e_{B 1}$ and $e_{B 2}$, two electrons which participate to the bond, denoted by $e_{1}$ and $e_{2}, N_{A}$ electrons which move in the field of the nucleus $n_{A}$ and do not participate to the bond, denoted by $e_{A i}$, and $N_{B}$ 


\section{Molecular Physics}

electrons which move in the field of the nucleus $n_{B}$ and do not participate to the bond, denoted by $e_{B j}$. The electrons $e_{A i}$ and $e_{B j}$ are shown in average positions in Fig. 4. We suppose that the molecule is symmetrical, and have $N_{A}=N_{B}$ and $i, j=3,4, \ldots, N_{A}$.

The calculation of the energetic values and geometric parameters of the diatomic molecule is performed in two stages, as follows.

1) In the first stage we calculate the values of $E_{b}, \sigma$ and $a$ with the aid of (A6), (A9) and (A10) in the Appendix. The method of computation originates in Refs. $[23,24]$, and in Appendix A we present an improved version of this method in the typical case of the simple bond of homonuclear diatomic molecules.

We use normalized quantities. The energies are normalized to $R_{\infty}$ and the distances to $2 a_{0}$, as follows: $\underline{E}_{b}=E_{b} / R_{\infty}, \underline{\sigma}=\sigma /\left(2 a_{0}\right)$ and $\underline{a}=a /\left(2 a_{0}\right)$. Taking into account these relations, together with the relations $R_{\infty}=\left(m K_{1}^{2}\right) /\left(2 \hbar^{2}\right)=$ $K_{1} /\left(2 a_{0}\right)$ (see Appendix A of Ref. [27]), (A6), (A9) and (A10) become

$$
\begin{gathered}
\underline{E}_{b}=-2 \frac{Z_{1}^{2}}{n_{1}^{2}}-\frac{8 Z_{1 M}}{\sqrt{9 \underline{a}^{2}+25 \underline{\sigma^{2}}}}+\frac{2}{\sqrt{9 \underline{a}^{2}+\underline{\sigma}^{2}}}+\frac{Z_{n A}^{2}}{2 \underline{\sigma}} \\
\underline{E}_{b}=-\frac{2 n_{1} \sqrt{Z_{1 M}}}{\left(\underline{a}^{2}+\underline{\sigma}^{2}\right)^{3 / 4}} \\
\underline{E}_{b}=-\frac{4 Z_{1 M}}{\sqrt{\underline{a}^{2}+\underline{\sigma}^{2}}}+\frac{1}{2 \underline{a}}+\frac{Z_{n A}^{2}}{2 \underline{\sigma}}
\end{gathered}
$$

This is a system of three equations with three unknowns: $\underline{E}_{b}, \underline{\sigma}$ and $\underline{a}$. In this case, taking into account the structure of the molecule (shown in Fig. 4) the expression of $Z_{1}$ must contain the screening coefficients due to the $e_{A i}$ electrons and it can be written:

$$
Z_{1}=Z_{A}-2 s_{1, A 1}-\sum_{i=3}^{N_{A}} s_{1, A i}
$$

where $s_{1, A 1}$ is approximated very well by $s_{31 e}$, calculated for atoms, while $s_{1, A i}$ is 
calculated with (9). The expression of $Z_{1 M}$ is modified, as follows:

$$
Z_{1 M}=Z_{A}-2-\sum_{i=3}^{N_{A}} s_{1, A i}
$$

Also, the expression of $Z_{n A}$ must be modified, in order to take into account the effect of the screening due to the electrons $e_{A i}$. This expression is deduced in Ref. [24] and it is given by Eq. (4.8) from that paper. With the notations from Fig. 4, where $r_{i n B}$ is the average distance between $n_{B}$ and $e_{A i}$ and $\alpha_{i}$ is the angle between the two lines which pass, respectively, through $n_{B}$ and $e_{A i}$ and through $n_{B}$ and $n_{A}$, this relation can be written, as follows

$$
Z_{n A}=Z_{A}-2-\sum_{i=3}^{N_{A}} \frac{(2 \sigma)^{2}}{r_{i n B}^{2}} \cos \alpha_{i}
$$

2) In the second stage, we calculate directly the normalized expression of the total energy, corresponding to the average positions of the electrons. We assume that the average positions of the electrons which do not participate to the bond are disposed in a configuration having maximum symmetry. The average normalized distance between the $e_{A i}$ electron and the nucleus is calculated from (12), which can be written:

$$
\underline{r}_{A i}=\frac{n_{A i}^{2}}{2 Z_{A i}}\left(1+\frac{e^{2}}{2}\right)
$$

where $Z_{A i}$ is the order number corresponding to the electron $e_{A i}$.

Taking into account the symmetry of the system, where $\underline{E}_{1}=\underline{E}_{2}, \underline{E}_{A i}=\underline{E}_{B i}$, $\underline{E}_{A 1}=\underline{E}_{A 2}=\underline{E}_{B 1}=\underline{E}_{B 2}, \underline{U}_{e_{1} n_{B}}=\underline{U}_{e_{2} n_{A}}, \underline{U}_{e_{A i} n_{B}}=\underline{U}_{e_{B i} n_{A}}$ and $\underline{U}_{e_{A i} e_{2}}=\underline{U}_{e_{B i} e_{1}}$, the normalized expression of the total energy can be written as follows:

$$
\begin{array}{r}
\underline{E}=2 \underline{E}_{1}+2 \sum_{i=3}^{N_{A}} \underline{E}_{A i}+4 \underline{E}_{A 1}+\underline{U}_{e_{1} e_{2}}+2 \underline{U}_{e_{1} n_{B}}+2 \sum_{i=3}^{N_{A}} \underline{U}_{e_{A i} e_{2}}+ \\
2 \sum_{i=3}^{N_{A}} \underline{U}_{e_{A i} n_{B}}+\sum_{i=3}^{N_{A}} \sum_{j=3}^{N_{B}} \underline{U}_{e_{A i} e_{B j}}+\underline{U}_{n_{A} n_{B}}+2 \underline{E}_{m 1 s}=-2 \frac{Z_{1}^{2}}{n_{1}^{2}}-2 \sum_{i=3}^{N_{A}} \frac{Z_{A i}^{2}}{n_{A i}^{2}}- \\
4 \frac{Z_{A 1}^{2}}{n_{A 1}^{2}}+\frac{1}{\left|\underline{\underline{r}}\left(e_{1}\right)-\underline{\underline{r}}\left(e_{2}\right)\right|}-\frac{2\left(Z_{A}-2\right)}{\left|\underline{\underline{r}}\left(e_{1}\right)-\underline{\underline{r}}\left(n_{B}\right)\right|}+2 \sum_{i=3}^{N_{A}} \frac{1}{\left|\underline{\underline{r}}\left(e_{A i}\right)-\underline{\bar{r}}\left(e_{2}\right)\right|}- \\
2 \sum_{i=3}^{N_{A}} \frac{Z_{A}-2}{\left|\underline{\underline{r}}\left(e_{A i}\right)-\underline{\bar{r}}\left(n_{B}\right)\right|}+\sum_{i=3}^{N_{A}} \sum_{j=3}^{N_{B}} \frac{1}{\left|\underline{\underline{r}}\left(e_{A i}\right)-\underline{\bar{r}}\left(e_{B j}\right)\right|}+\frac{\left(Z_{A}-2\right)^{2}}{\left|\underline{\underline{r}}\left(n_{A}\right)-\underline{\bar{r}}\left(n_{B}\right)\right|}+ \\
2 \frac{Z_{A 1}^{3 / 2}}{8 n_{A 1}^{3}}
\end{array}
$$

where $\underline{\bar{x}}\left(e_{1}\right)$ and $\underline{\bar{x}}\left(n_{A}\right)$ are, respectively, the average normalized position vectors of the electron $e_{1}$ and nucleus $n_{A}, \underline{U}_{e_{1} e_{2}}$ is the normalized potential energy of electrostatic interaction between the electron $e_{1}$ and nucleus $e_{2}$, and so on. The 
expressions of the order numbers are $Z_{1}$ given by (37) and

$$
\begin{gathered}
Z_{A 1}=Z_{A}-s_{A 1, A 2}-s_{A 1,1}-\sum_{i=3}^{N_{A}} s_{A 1, A i} \\
Z_{A i}=Z_{A}-2 s_{A i, A 1}-s_{A i, 1}-\sum_{j=3 ; i \neq j}^{N_{A}} s_{A i, A j} \text { for } i \geq 3
\end{gathered}
$$

where $s_{A i, A 1}$ are, with good approximaton, equal to $s_{31 e}$, calculated in the case of atoms, while $s_{A 1,1}$ and $s_{A 1, A i}$ are equal to $s_{13 e}$. The coefficients $s_{1, A i}, s_{A i, A j}$ and $s_{A 1, A 2}$ are calculated with (9).

The first, second and third terms of the second member in (41) are the total normalized energies of the electrons which move in the field of the same nucleus. Their significance is identical to the significance of electron energies in an atomic system (see Section 2), taking into account that new indexes are introduced in the case of molecular systems, as it is shown in Section 3. We recall that the components of the energies, denoted by $E_{1}, E_{A i}$ or $E_{A 1}$, are given by the equations (4) and (5). The other terms represent the normalized electrostatic interaction energies, corresponding to interactions at distance between electrons that do not move in the field of the same nucleus, between electrons and nuclei which are situated at distance from them, and between nuclei, having order numbers which include the screening effects of the $1 s$ electrons. The last term is given by (11) and represents the normalized correction energies due to the spin magnetic interaction of the $1 s$ electrons. An analysis of equation (41) shows that all the components of the interaction energies are taken into account.

In virtue of the hypothesis (h5), the kinetic energy of the nuclei is zero. It follows that the distance between nuclei, denoted by $r_{0}$ and calculated with the relation

$$
r_{0}=2 \sigma
$$

corresponds to values of the nuclei velocities of zero. On the other hand, the experimental value of the distance between nuclei, denoted by $r_{e}$, corresponds to the minimum of the Morse curve [34]. The theory of the harmonic oscillation of the nuclei shows that this minimum corresponds to maximum velocities of the nuclei. It follows that $r_{0}$ is different from $r_{e}$ because the first corresponds to zero velocities of the nuclei, while the last corresponds to the maximum velocities of the nuclei.

The normalized experimental values of the total energy are obtained with the aid of the relation

$$
\underline{E}_{e x p}=-2 \underline{S}_{E i A}-\underline{D}_{0}^{0}
$$

where $S_{E i A}$ and $D_{0}^{0}$ are, respectively, the sum of the ionization energies of the atom $A$ and the observed dissociation energy. The values of $S_{E i A}$ and $D_{0}^{0}$ are taken, respectively, from Refs. [35] and [36]. Since our calculation method is based on the central field approximation under the assuption that a given electron moves in the averaged field of the other electrons, in Table 1 we will compare our theoretical results, with similar results reported in literature [29], obtained with the aid of the Hartree-Fock method.

The calculations are made with the aid of Mathematica 7, the absolute error being of the order $10^{-16}$. 


\section{Molecular Physics}

Molecular Physics

1

3

4

5

7

9

10

11

12

13

14

15

16

17

18

19

20

21

Table 1. Normalized values of the total energies $\underline{E}$, calculated for homonuclear and heteronuclear diatomic molecules. For comparison, are given the best two values of the normalized values of the total energies from CCCBDB [29], denoted by $\underline{E}_{H F}$, which are calculated with the aid of the HartreeFock method and the corresponding experimental values $\underline{E}_{\text {exp. }}$. All the values are given in Rydbergs.

\begin{tabular}{lccc}
\hline Molecule & $\underline{E}$ & $\underline{E}_{H F}[29]$ & $\underline{E}_{\exp }$ \\
\hline $\mathrm{Li}_{2}$ & -30.0919 & -29.73625 & -29.9896 \\
& & -29.736246 & \\
$\mathrm{Be}_{2}$ & -58.7676 & -58.486596 & -58.8396 \\
& & -58.48645 & \\
$\mathrm{~B}_{2}$ & -98.3341 & -98.182662 & -98.8574 \\
& & -98.18259 & \\
$\mathrm{C}_{2}$ & -151.148 & -150.804028 & -151.883 \\
& & -150.803916 & \\
$\mathrm{LiH}$ & -16.1821 & -15.967824 & -16.1348 \\
& & -15.96775 & \\
$\mathrm{BeH}$ & -30.4238 & -30.300286 & -30.4872 \\
& & -30.300248 & \\
$\mathrm{BH}$ & -50.4587 & -50.256246 & -50.5691 \\
$\mathrm{CH}$ & -76.6349 & -76.561468 & -76.968 \\
& & -76.560994 & \\
\hline
\end{tabular}

Table 2. Coordinates of average positions of particles, effective screening coefficients, effective order numbers and solutions of the equations describing the behavior of $\mathrm{Li}_{2}$ and $B e_{2}$ molecules.

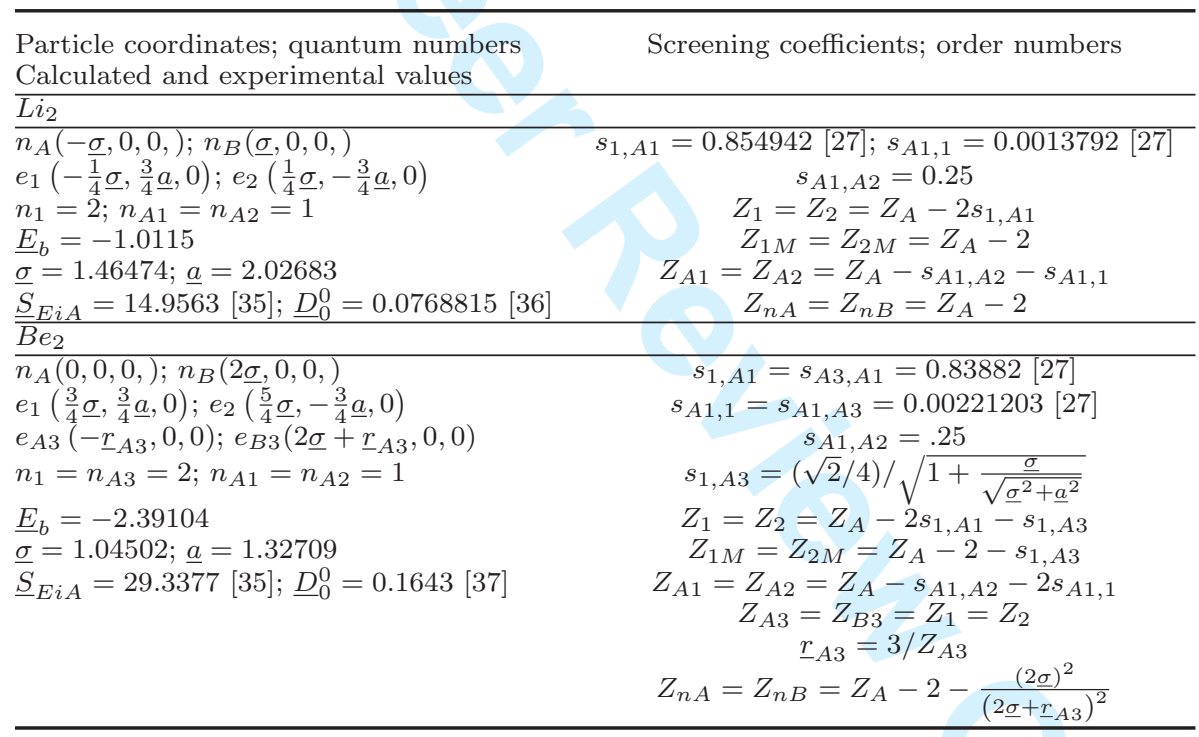

\section{Typical examples of applications}

\section{a) $L i_{2}$ molecule.}

This molecule contains only valence electrons that participate to the bond. Its structure is shown in Fig. 1. We follow the algorithm presented in Section 4 and calculate the screening coefficients, order numbers and then solve the system (34)(36). All these values are given in Table 2. The screening coefficients between the $1 s$ electrons and the $e_{1}$ and $e_{2}$ electrons, namely $s_{1, A 1}$ and $s_{A 1,1}$ are identical, respectively, to $s_{31 e}$ and $s_{13 e}$, which have been calculated in Ref. [27] for the $1 s^{2} 2 s$ state of lithium atom. The coefficient $s_{A 1, A 2}$ is calculated from equation (9). Since $2 s$ valence electrons participate to the bond, we have $n_{1}=2$. Also, we have $n_{A 1}=1$ because this number corresponds to $1 s$ electrons. We solve the system (34)-(36) in order to obtain $\underline{E}_{b}, \underline{\sigma}$ and $\underline{a}$. 
Table 3. Values of $r_{0}$ calculated in this paper compared with the experimental values of $r_{e}$ [29], and also with the range of values for $r_{e H F}$ calculated using Hartree-Fock methods [29]. All the values are given in angstroms.

\begin{tabular}{lccc}
\hline Molecule & $r_{0}$ & $r_{e}$ & $r_{e H F}$ \\
\hline $\mathrm{Li}_{2}$ & 3.10043 & 2.673 & $2.696-2.816$ \\
$\mathrm{Be}_{2}$ & 2.212 & 2.460 & $1.780-2.049$ \\
$\mathrm{~B}_{2}$ & 1.7883 & 1.590 & $1.512-1.562$ \\
$\mathrm{C}_{2}$ & 1.35694 & 1.243 & $1.240-1.308$ \\
$\mathrm{LiH}$ & 1.84635 & 1.5957 & $1.511-1.636$ \\
$\mathrm{BeH}$ & 1.4389 & 1.3426 & $1.301-1.356$ \\
$\mathrm{BH}$ & 1.21642 & 1.2324 & $1.213-1.232$ \\
$\mathrm{CH}$ & 1.07572 & 1.1199 & $1.111-1.127$ \\
\hline
\end{tabular}

Since in this case $E_{b}$ has the significance of the total energy from which the energies of the $1 s$ electrons are subtracted, we have:

$$
\underline{E}=\underline{E}_{b}+4 \underline{E}_{A 1}+2 \underline{E}_{m 1 s}=\underline{E}_{b}-4 \frac{Z_{A 1}^{2}}{n_{A 1}^{2}}+2 \frac{Z_{A 1}^{3 / 2}}{8 n_{A 1}^{3}}
$$

The values of $r_{0}$, calculated from (44), and the experimental value of the distance between nuclei, denoted by $r_{e}$ and taken from Ref. [29], are given in angstroms in Table 3. Table 3 also gives the range of values for the distances between nuclei, denoted by $r_{e H F}$, calculated with the Hartree-Fock method and taken from [29].

The normalized value of the total energy, $\underline{E}$, obtained from equation (46), together with the experimental value, denoted by $\underline{E}_{\text {exp }}$, are presented in Table 1 . We also present in this table the best two values of the normalized total energies, calculated with the aid of the Hartree-Fock method, denoted by $\underline{E}_{H F}$, which are taken from $C C C B D B$ [29]. The experimental values of the total energy are obtained with the aid of equation (45), where $S_{E i A}$ and $D_{0}^{0}$ which are taken, respectively, from Refs. [35] and [36], are given in Table 2. The analysis of the data from Table 1 shows that the value of $\underline{E}$ is closer to the experimental value, $\underline{E}_{\text {exp }}$, than the values of $\underline{E}_{H F}$. The relative errors in the two cases are, respectively, $\left|\left(\underline{E}-\underline{E}_{\text {exp }}\right) / \underline{E}_{\text {exp }}\right|=0.003$ and $\left|\left(\underline{E}_{H F}-\underline{E}_{\text {exp }}\right) / \underline{E}_{\text {exp }}\right|=0.008$.

\section{b) $\mathrm{Be}_{2}$ molecule.}

The structure of this molecule is shown in Fig. 5. This molecule contains two electrons, denoted by $e_{A 3}$ and $e_{B 3}$, which do not participate to the bond. The geometrical structure of the molecule results from the system (34)-(36), where the expressions of $Z_{1}, Z_{1 M}, Z_{n A}$ and the value of $n_{1}$ are given in Table 2 . The screening coefficients between the $1 s$ electrons and the $e_{1}, e_{2}, e_{A 3}$ and $e_{B 3}$ electrons, namely $s_{1, A 1}, s_{A 1,1}, s_{A 3, A 1}, s_{A 1, A 3}$, and so on, are identical, respectively to $s_{31 e}$ and $s_{13 e}$, which have been calculated in Ref. [27] for the $1 s^{2} 2 s^{2}$ state of beryllium atom. The coefficient $s_{1, A 3}$ is calculated with (9). This calculation reduces to the evaluation of the angle between the two lines which pass, respectively, through nucleus $n_{A}$ and electron $e_{1}$ and through nucleus $n_{A}$ and electron $e_{A 3}$, with the aid of the coordinates of the particles, which are shown in Table 2. All the other necessary data are given in Table 2.

The normalized value of the total energy is calculated with the aid of equation (41), and can be written for $B e_{2}$ molecule in the following form, taking into account the symmetry of the system (namely $\underline{E}_{1}=\underline{E}_{2}, \underline{E}_{A 3}=\underline{E}_{B 3}, \underline{E}_{A 1}=\underline{E}_{A 2}=\underline{E}_{B 1}=$ 


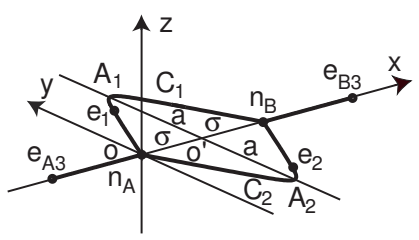

Figure 5. Structure of $B e_{2}$ molecule.

$$
\begin{aligned}
\left.\underline{E}_{B 2}, \underline{U}_{e_{1} n_{B}}=\underline{U}_{e_{2} n_{A}}, \underline{U}_{e_{A 3} e_{2}}=\underline{U}_{e_{B 3} e_{1}} \text { and } \underline{U}_{e_{A 3} n_{B}}=\underline{U}_{e_{B 3} n_{A}}\right): \\
\underline{E}=2 \underline{E}_{1}+2 \underline{E}_{A 3}+4 \underline{E}_{A 1}+\underline{U}_{e_{1} e_{2}}+2 \underline{U}_{e_{1} n_{B}}+2 \underline{U}_{e_{A 3} e_{2}}+2 \underline{U}_{e_{A 3} n_{B}}+(47) \\
\underline{U}_{e_{A 3} e_{B 3}}+\underline{U}_{n_{A} n_{B}}+2 \underline{E}_{m 1 s}=-2 \frac{Z_{1}^{2}}{n_{1}^{2}}-2 \frac{Z_{A 3}^{2}}{n_{A 3}^{2}}-4 \frac{Z_{A 1}^{2}}{n_{A 1}^{2}}+\frac{1}{2 \sqrt{\left(\frac{1}{4} \underline{\sigma}\right)^{2}+\left(\frac{3}{4} \underline{a}\right)^{2}}}- \\
\frac{2\left(Z_{A}-2\right)}{\sqrt{\left(\frac{5}{4} \underline{\sigma}\right)^{2}+\left(\frac{3}{4} \underline{a}\right)^{2}}}+\frac{2}{\sqrt{\left(\frac{5}{4} \underline{\sigma}+\underline{r}_{A 3}\right)^{2}+\left(\frac{3}{4} \underline{a}\right)^{2}}-\frac{2\left(Z_{A}-2\right)}{2 \underline{\sigma}+\underline{r}_{A 3}}}+ \\
\frac{1}{2 \underline{\sigma}+2 \underline{r}_{A 3}}+\frac{\left(Z_{A}-2\right)^{2}}{2 \underline{\sigma}}+2 \frac{Z_{A 1}^{3 / 2}}{8 n_{A 1}^{3}}
\end{aligned}
$$

In this case $\underline{E}_{\exp }$ is obtained from equation (45), where $S_{E i A}$ is taken from Ref. [35], but the value of $D_{0}^{0}$ is obtained from Fig. 6-2 of Ref. [37] (Ref. [36] does not contain this data). The values of $r_{0}$ and $r_{e}$ are given in Table 3, while $\underline{E}, \underline{E}_{H F}$ and $\underline{E}_{\text {exp }}$ are shown in Table 1 . The relative errors corresponding to $\underline{E}$ and $\underline{E}_{H F}$ are, respectively, $\left|\left(\underline{E}-\underline{E}_{e x p}\right) / \underline{E}_{e x p}\right|=0.001$ and $\left|\left(\underline{E}_{H F}-\underline{E}_{\exp }\right) / \underline{E}_{\exp }\right|=0.006$.

\section{c) Other molecules.}

The procedure for calculating the energetic values of $B_{2}, C_{2}, \mathrm{LiH}, \mathrm{BeH}, \mathrm{BH}$ and $\mathrm{CH}$ molecules is almost identical to that shown in the above examples. The values of $\underline{E}, \underline{E}_{H F}$ and $\underline{E}_{e x p}$, corresponding to these molecules are shown also in Table 1 . The specific differences, referring to these molecules are given briefly below.

The $B_{2}$ molecule contains four electrons, denoted by $e_{A 3}, e_{A 4}, e_{B 3}$ and $e_{B 4}$ which do not participate to the bond. Taking into account this difference, the procedure for calculating the energetic values is identical to that for the $\mathrm{Be}_{2}$ molecule.

We have analyzed the $C_{2}$ molecule in three cases: when the bond is simple, double and triple and found that the total energy is minimum in the case of the double bond, resulting that the real bond of the $C_{2}$ molecule is double. This result is identical to that obtained by pure quantum evaluations (see, for example pag. 97 of Ref. [31]). In Table 1 it is shown the value of $\underline{E}$ corresponding to the $C_{2}$ molecule with double bond, together with $\underline{E}_{H F}$ and $\underline{E}_{\text {exp }}$. We have found also that the symmetry properties of the $C_{2}$ molecules, corresponding to simple, double and triple bonds are identical, respectively, to the experimental symmetry properties of the ethane, ethylene and acetylene molecules, namely $\mathrm{C}_{2} \mathrm{H}_{6}, \mathrm{C}_{2} \mathrm{H}_{4}$ and $\mathrm{C}_{2} \mathrm{H}_{2}$ (see Ref. [29]). This explains why adding 6, 4 and, respectively, 2 hydrogen atoms to the structures of $C_{2}$ with simple, double and triple bonds leads to the formation of the $\mathrm{C}_{2} \mathrm{H}_{6}, \mathrm{C}_{2} \mathrm{H}_{4}$ and, respectively, $\mathrm{C}_{2} \mathrm{H}_{2}$ molecules. Also, our calculations explain 
the property that the length of the bond decreases as the bond order increases.

We have analyzed the following heteronuclear diatomic molecules: $\mathrm{LiH}, \mathrm{BeH}$, $B H$ and $C H$. The model of ionic bond, as it results from Fig. 3(a), is in agreement with the experimental data for $\mathrm{LiH}, \mathrm{BeH}$, while the model of covalent bond, which is shown in Fig. 3(b), is in agreement with the experimental data only for $B H$ and $\mathrm{CH}$. For example, if we apply the covalent model in the case of $\mathrm{LiH}$, it will lead to a symmetrical molecule, for which $\sigma_{1}=\sigma_{2}$, which is in strong disagreement with the fact that this molecule has a strong ionic character [31]. Outside of these differences, the procedure of calculation of the energetic values is almost identical to that shown in the above examples.

All the details of the computations and the Mathematica 7 scripts used for all the molecules are given in the Supplementary Online Material for this article.

\section{Conclusions}

We presented a semiclassical central field method for the calculation of the energetic values of the diatomic molecules $\mathrm{Li}_{2}, \mathrm{Be}_{2}, \mathrm{~B}_{2}, \mathrm{C}_{2}, \mathrm{LiH}, \mathrm{BeH}, \mathrm{BH}$ and $\mathrm{CH}$, which is based on an exact theoretical connection between the geometric elements of the wave described by the Schrödinger equation and the Hamilton-Jacobi equation, written for the same system. This connection was proved and was checked on atomic systems in previous papers. The accuracy of our method is comparable with the accuracy of the standard central field Hartree-Fock method, for both, atomic systems and diatomic molecules. Our method reveals a series of other properties of the diatomic molecules, such as the fact that the bond of $\mathrm{C}_{2}$ is double, or $\mathrm{LiH}$ has a strong ionic character, which is in agreement with experimental measurements.

\section{Appendix A. Analytical calculation method for geometric parameters in the typical case of the simple bond of homonuclear diatomic molecules.}

We consider a molecule composed of two fixed identical nuclei, denoted by $n_{A}$ and $n_{B}$, four $1 s$ electrons and two valence electrons which participate to the bond, denoted by $e_{1}$ and $e_{2}$. The Cartesian coordinates of the nuclei are, respectively, $n_{A}(-\sigma, 0,0)$ and $n_{B}(\sigma, 0,0)$ and their order number is equal to $Z_{A}$. The $C_{1}$ and $C_{2}$ curves, which correspond to the bond electrons are situated in the plane $(x, y)$, as shown in Fig. 1. In Subsection 3.1 we have shown that each curve is composed of two quasilinear ellipses, denoted by $C_{a A}$ and $C_{a B}$. We assume that the kinetic energies of the electrons positioned at points $A_{1}(0, a, 0)$ and $A_{2}(0,-a, 0)$ are negligible as compared to the total energy. The electrons are situated in average positions in Fig. 1, and their coordinates are $e_{1}[-(1 / 4) \sigma,(3 / 4) a, 0]$ and $e_{2}[(1 / 4) \sigma,-(3 / 4) a, 0]$, in agreement with (12), for $e \cong 1$ and $r_{M}=\sqrt{a^{2}+\sigma^{2}}$. In this case $E_{b}$ has the significance of the total energy from which the energies of the $1 s$ electrons are subtracted. The three quantities $E_{b}, \sigma$ and $a$ are calculated with the aid of the following three equations [23, 24].

1) The first relation. This relation results from the central field approximation. It results in a similar manner as in the case of atoms $[27,28]$ : we have to write the expression of the energy of the electron $e_{1}$, denoted by $E_{1}$, and to consider the approximation of the central field. We have $E_{1}=T_{1}+U_{e_{1} n_{A}}$, where $T_{1}$ is the kinetic energy of the electron $e_{1}$ and $U_{e_{1} n_{A}}$ is the electrostatic energy of interaction between the electron $e_{1}$ and the nucleus $n_{A}$, which includes the screening effect of the $1 s$ electrons. Taking into account the symmetry of the system (from where we 
have $\left.T_{1}=T_{2}, U_{e_{1} n_{A}}=U_{e_{2} n_{B}}, U_{e_{1} n_{B}}=U_{e_{2} n_{A}}\right)$ and the expression of the energy $E_{b}$, we have

$$
E_{1}=T_{1}+U_{e_{1} n_{A}}=\frac{E_{b}}{2}-U_{0} \text { with } U_{0}=U_{e_{1} n_{B}}+\frac{1}{2}\left(U_{e_{1} e_{2}}+U_{n_{A} n_{B}}\right)
$$

The significance of the above electrostatic terms is specified by their notations (for example, $U_{e_{1} n_{B}}$ is the potential energy of electrostatic interaction between the electron $e_{1}$ and nucleus $n_{B}$, and so on), and the screening effect of the $1 s$ electrons is taken into account through the terms $U_{e_{1} n_{B}}$ and $U_{n_{A} n_{B}}$. In these terms, which refer at the interaction at distance between electrons and nuclei, and, respectively, between nuclei, the screening coefficient due to the $1 s$ electrons is equal to unity. The expressions of these terms are (see Fig. 1): $U_{e_{1} n_{B}}=K_{1} Z_{1 M} / s_{1}^{\prime}$ and $U_{n_{A} n_{B}}=$ $K_{1} Z_{n A}^{2} /(2 \sigma)$, where the corresponding order numbers are

$$
Z_{1 M}=Z_{A}-2 \text { and } Z_{n A}=Z_{A}-2
$$

Since $U_{0}$ is a relatively small quantity, we consider its averaged value, denoted by $U_{0 m}$, calculated for the average positions of the electrons. In this case the expression $E_{1}=T_{1}+U_{e_{1} n_{A}}=\frac{E_{b}}{2}-U_{0 m}$ represents the constant energy of a hydrogenoid system, resulting that the electron $e_{1}$ moves in the central field of the nucleus in an averaged field of the other electron. We apply the quantization relation from (2), which is $\Delta_{C_{1 A}} S_{01}=n_{1} h$, for the curve $C_{1 A}$ and, in virtue of the relations shown in Appendix A of Ref. [27], we have:

$$
E_{1}=\frac{E_{b}}{2}-U_{0 m}=-\frac{Z_{1}^{2} R_{\infty}}{n_{1}^{2}}
$$

where

$$
U_{0 m}=-\frac{4 K_{1} Z_{1 M}}{\sqrt{9 a^{2}+25 \sigma^{2}}}+\frac{K_{1}}{\sqrt{9 a^{2}+\sigma^{2}}}+\frac{K_{1} Z_{n A}^{2}}{4 \sigma}
$$

and

$$
Z_{1}=Z_{A}-2 s_{1, A 1}
$$

From (A3), (A4) obtain

$$
E_{b}=-2 \frac{Z_{1}^{2} R_{\infty}}{n_{1}^{2}}-\frac{8 K_{1} Z_{1 M}}{\sqrt{9 a^{2}+25 \sigma^{2}}}+\frac{2 K_{1}}{\sqrt{9 a^{2}+\sigma^{2}}}+\frac{K_{1} Z_{n A}^{2}}{2 \sigma}
$$

2) The second relation. This relation results from the virial theorem. Taking into account that the curve $C_{1}$ is symmetrical, the virial theorem can be written

$$
E_{b}=-\tilde{T}=-\frac{1}{\tau} \int_{\tau} m v_{1}^{2} d t=-\frac{\Delta_{C_{1 A}} S_{01}}{\tau}=-\frac{n_{1} h}{\tau}
$$

where $\tilde{T}$ is the average value of the total kinetic energy and $\tau$ is the period corresponding to the curve $C_{1 A}$. Since $\tau=2 \int_{0}^{r_{M}} d s_{1} / v_{1}$ where $v_{1}=0$ and $T_{1}=0$ for $s_{1}=r_{M}$, it results that the domain in the vicinity of the point $A_{1}$, which is relatively far from nucleus, has a big weight in the calculation of the integral. In this case we have $U_{e_{1} n_{A}} \cong-K_{1} Z_{1 M} / s_{1}$, and from (A1) we obtain 
$E_{b} / 2-U_{0}=T_{1}-K_{1} Z_{1 M} / s_{1}=-K_{1} Z_{1 M} / r_{M}$, or $T_{1}=K_{1} Z_{1 M} / s_{1}-K_{1} Z_{1 M} / r_{M}$. The expression of $\tau$ becomes:

$$
\tau=2 \int_{0}^{r_{M}} \frac{d s_{1}}{\sqrt{\frac{2 T_{1}}{m}}}=\sqrt{2 m} \int_{0}^{r_{M}} \frac{d s_{1}}{\sqrt{\frac{K_{1} Z_{1 M}}{s_{1}}-\frac{K_{1} Z_{1 M}}{r_{M}}}}=\pi \sqrt{\frac{m}{2 K_{1} Z_{1 M}}} r_{M}^{\frac{3}{2}}
$$

Introducing the expression of $r_{M}$, and taking into account (A7), the above relation becomes

$$
E_{b}=-\frac{n_{1} h \sqrt{2 K_{1} Z_{1 M}}}{\pi \sqrt{m}\left(\sigma^{2}+a^{2}\right)^{\frac{3}{4}}}
$$

3) The third relation. This relation results from the equation of the energy $E_{b}$, when the electrons are situated at maximum distance from the nuclei, respectively, in the points $A_{1}(0, a, 0)$ and $A_{2}(0,-a, 0)$, as follows:

$$
E_{b}=4 U_{e_{1} n_{A}}+U_{e_{1} e_{2}}+U_{n_{A} n_{B}}=-\frac{4 K_{1} Z_{1 M}}{\sqrt{\sigma^{2}+a^{2}}}+\frac{K_{1}}{2 a}+\frac{K_{1} Z_{n A}^{2}}{2 \sigma}
$$

The calculations of the geometric parameters of the bond in the cases of the double and triple covalent bonds of homonuclear molecules and in the cases of ionic and covalent bonds in heteronuclear molecules are similar to the calculation presented in this Appendix. These calculations are shown in details in the Supplementary Online Material.

\section{Acknowledgements}

This work was done in the frame of the basic research program of the National Institute for Laser, Plasma and Radiation Physics, entitled "Nucleus Program."

\section{References}

[1]A. M. Ozorio de Almeida, Phys. Reports 295, 265 (1998).

[2] M. F. Herman, Annu. Rev. Phys. Chem. 45, 83 (1994).

[3] C. C. Martens, A. Donoso, and Y. Zheng, in Multidimensional Quantum Dynamics with Trajectories edited by D. Shalashilin and M. P. de Miranda (CCP6, Daresbury, 2009).

[4] E. Roman and C. C. Martens, J. Chem. Phys 122, 174107 (2004).

[5] M. Thoss and G. Stock, Phys. Rev. A 59, 64 (1999).

6] M. Gryzynski, Phys. Lett. 44A, 131 (1973).

[7]R. G. Pearson, Proc. Natl Acad. Sci. USA 784002 (1981).

[8] P. V. Coveney and M. S. Child, J. Phys. B: At. Mol. Phys. 17319 (1984).

[9] M. Gryzynski, Phys. Lett. A 123, 170 (1987).

[10]D. L. Nascimento and A. L. A. Fonseca, Int. J. of Quantum Chem. 95274 (2003).

11]D. L. Nascimento, A. L. A. Fonseca and O. Portilho, Int. J. of Quantum Chem. 103, 505 (2005).

[12]C.-D. Yang, Int. J. of Quantum Chem. 1061620 (2006).

[13]R. A. Marcus, Chem. Soc. Faraday Discuss. 5534 (1973).

[14] W.A. Eastes and R. A. Marcus, J. Chem. Phys. 614301 (1974)

[15]D. W. Noid and R. A. Marcus, J. Chem. Phys. 622119 (1975).

[16] G. S. Ezra, K. Richter, G. Tanner and D. Wintgen, J. Phys. B 24 L413 (1991).

[17]K. Richter and D. Wintgen, Phys. Rev. Lett. 651965 (1990).

[18] J. Muller, J. Burgborfer, Phys. Rev. Lett. 702375 (1993).

[19]P. Gaspard, Phys. Rev. A 4854 (1993).

[20]K. G. Gay, J. Chem. Phys. 1004377 (1994).

[21] W. H. Miller, Science 233171 (1986).

[22] A. Popa, J. Phys. Soc. of Jpn 67, 2645 (1998).

[23] A. Popa, J. Phys. Soc. of Jpn 68, 763 (1999).

[24] A. Popa, J. Phys. Soc. of Jpn 68, 2923 (1999).

[25] A. Popa, J. Phys. A: Math. and General 36, 7569 (2003). 
1

[26] A. Popa, J. Chem. Phys. 122, 244701 (2005).

[27] A. Popa, Eur. Phys. J. D 49, 279 (2008).

[28] A. Popa, Eur. Phys. J. D 54, 575 (2009).

29] The Computational Chemistry Comparison and Benchmark Database (CCCBDB). http://cccbdb.nist.gov/

[30]L. Pauling, General Chemistry (W. H. Freeman and Company, San Francisco, 1970).

[31]C. A. Coulson, Valence (Clarendon Press, Oxford, 1953).

[32] A. Luis, Phys. Rev. A 67, 024102 (2003).

33] M. D. Poulsen and L. B. Madsen, Phys. Rev. A 72, 042501 (2005)

[34] G. Hertzberg, Molecular Spectra and Molecular Structure. I. Spectra of Diatomic Molecule (Van Nostrand, New York, 1950).

[35]D. R. Lide (ed) CRC Handbook of Chemistry and Physics (CRC Press, Boca Raton, Florida 2003).

[36] K. P. Huber and G. Hertzberg, Molecular Spectra and Molecular Structure. IV. Constants of Diatomic Molecules (Van Nostrand, New York, 1979).

[37]J. C. Slater, Quantum Theory of Molecules and Solids. Vol. 1 (McGraw Hill, New York, 1963). 


\title{
SUPPLEMENTARY ONLINE MATERIAL
}

\author{
for the paper
}

\section{Unconventional semiclassical method for calculating the energetic val- ues of diatomic molecules}

\author{
Alexandru Popa
}

National Institute for Laser, Plasma and Radiation Physics, Laser Department, P.O. Box MG-36, Bucharest, Romania 077125

In this material we extend the calculation of the geometric parameters of the bond, which has been presented in the Appendix A of the paper, to the cases of the double and triple covalent bonds of homonuclear molecules and to the cases of ionic and covalent bonds in heteronuclear molecules. We present also detailed calculations of the molecules properties in the cases of $B_{2}, C_{2}, \mathrm{LiH}, \mathrm{BeH}, \mathrm{BH}$ and $\mathrm{CH}$ molecules, together with Mathematica 7 scripts containing the calculations necessary for all the molecules which have been analyzed in paper and in supplementary material. The references are those from the paper.

S1. Calculation of the geometric parameters in the particular cases of the double and triple covalent bonds of homonuclear molecules and of ionic and covalent bonds in heteronuclear molecules.

\section{S1.1. Covalent double bond of homonuclear molecules}

1) The first relation. The procedure is identical to that for simple bonds, which has been presented in Appendix A of the paper. We need, however, to take into account that four electrons participate in this case to the bond. Their average positions are $e_{1}[-(1 / 4) \sigma,(3 / 4) a, 0], e_{2}[-(1 / 4) \sigma,-(3 / 4) a, 0], e_{3}[(1 / 4) \sigma, 0,(3 / 4) a]$ and $e_{4}[(1 / 4) \sigma, 0,-(3 / 4) a]$, as shown in Fig.2(a) from the paper. In this case, taking into account the symmetry relations $T_{1}=T_{2}=T_{3}=T_{4}, U_{e_{1} n_{A}}=$ $U_{e_{2} n_{A}}=U_{e_{3} n_{B}}=U_{e_{4} n_{B}}, U_{e_{1} n_{B}}=U_{e_{2} n_{B}}=U_{e_{3} n_{A}}=U_{e_{4} n_{A}}$ and $U_{e_{1} e_{3}}=U_{e_{1} e_{4}}=$ $U_{e_{2} e_{3}}=U_{e_{2} e_{4}}$, (A1) from paper becomes: $E_{1}=T_{1}+U_{e_{1} n_{A}}=\frac{E_{b}}{4}-U_{0}$ with $U_{0}=U_{e_{1} n_{B}}+\frac{1}{4}\left(4 U_{e_{1} e_{3}}+U_{n_{A} n_{B}}\right)$. A procedure identical to the one presented in Appendix A of the paper, while taking into account the quantization relation, leads to the equation $E_{1}=-Z_{1}^{2} R_{\infty} / n_{1}^{2}$ where

$$
Z_{1}=Z_{A}-2 s_{1, A 1}-s_{1,2} \text { and } s_{1,2}=s_{2,1}=\frac{\sqrt{\sigma^{2}+a^{2}}}{4 a}
$$

The screening coefficient $s_{1,2}$ is calculated with (9) from paper. We have also

$$
Z_{1 M}=Z_{A}-2-s_{1,2}
$$

In virtue of the above relations, the second term of the second member of (A6) from paper corresponds to $U_{e 1 n B}+U_{e 2 n B}+U_{e 3 n A}+U_{e 4 n A}=4 U_{e 1 n B}$ instead of $2 U_{e 1 n B}$ obtained in the case of the single bond. We can further write: $4 U_{e 1 n B}=$ $\frac{4 K_{1}\left(Z_{B}-2\right)}{\sqrt{(5 \sigma / 4)^{2}+(3 a / 4)^{2}}}=\frac{16 K_{1}\left(Z_{1 M}+s_{1,2}\right)}{\sqrt{25 \sigma^{2}+9 a^{2}}}$. The third term of the second member of (A6) from paper corresponds to the interactions between the bond electrons $e_{1}, e_{2}$ and the bond electrons $e_{3}, e_{4}$, and, because of symmetry, it corresponds to $4 U_{e_{1} e_{3}}$, 
instead of $U_{e 1 e 2}$ obtained in the case of simple bond. With these modifications, the correspondent of (A6) from paper, in the case of double bond is:

$$
E_{b}=-4 \frac{Z_{1}^{2} R_{\infty}}{n_{1}^{2}}-\frac{16 K_{1}\left(Z_{1 M}+s_{1,2}\right)}{\sqrt{9 a^{2}+25 \sigma^{2}}}+\frac{8 K_{1}}{\sqrt{\sigma^{2}+4.5 a^{2}}}+\frac{K_{1} Z_{n A}^{2}}{2 \sigma}
$$

2) The second relation. Since there are four bond electrons, the virial relation must be modified, namely instead of (A7) from paper, we have $E_{b}=-2 n_{1} h / \tau$, from which it follows that the second relation, i.e. (A9) from paper, becomes:

$$
E_{b}=-\frac{2 n_{1} h \sqrt{2 K_{1} Z_{1 M}}}{\pi \sqrt{m}\left(\sigma^{2}+a^{2}\right)^{\frac{3}{4}}}
$$

3) The third relation. This relation results directly, taking into account that the first term from the second member represents the sum of the interactions between the bond electrons $e_{1}, e_{2}, e_{3}, e_{4}$, (situated, respectively, at points $\left.A_{1}, A_{2}, A_{3}, A_{4}\right)$ and nuclei, and the second term represents the sum of the interaction between all the bond electrons, corresponding to these positions. The third relation, (A10) from paper must be rewritten as follows:

$$
E_{b}=-\frac{8 K_{1}\left(Z_{1 M}+s_{1,2}\right)}{\sqrt{\sigma^{2}+a^{2}}}+\frac{K_{1}}{a}+\frac{2 \sqrt{2} K_{1}}{a}+\frac{K_{1} Z_{n A}^{2}}{2 \sigma}
$$

\section{S1.2. Covalent triple bond of homonuclear molecules}

The solution is similar to that for simple bonds. We have to take into account that, in this case, six electrons participate to the bond. Their average positions are $e_{1}\left[-\frac{1}{4} \sigma, \frac{3}{4} a, 0\right], e_{2}\left[-\frac{1}{4} \sigma,-\frac{3}{4} \cdot \frac{1}{2} a, \frac{3}{4} \cdot \frac{\sqrt{3}}{2} a\right], e_{3}\left[-\frac{1}{4} \sigma,-\frac{3}{4} \cdot \frac{1}{2} a,-\frac{3}{4} \cdot \frac{\sqrt{3}}{2} a\right], e_{4}\left[\frac{1}{4} \sigma,-\frac{3}{4} a, 0\right]$, $e_{5}\left[\frac{1}{4} \sigma, \frac{3}{4} \cdot \frac{1}{2} a,-\frac{3}{4} \cdot \frac{\sqrt{3}}{2} a\right]$ and $e_{6}\left[\frac{1}{4} \sigma, \frac{3}{4} \cdot \frac{1}{2} a, \frac{3}{4} \cdot \frac{\sqrt{3}}{2} a\right]$, as shown in Fig.2(b) from the paper. The analysis in this case is analogues to the analysis of the simple and double bonds. The difference from these two previous cases is that we have to take into account that six bond electrons participate to the triple bond instead of four (double bond) or two (simple bond) electrons. It easy to see that the three relations, in the case of the triple bond, become:

$$
\begin{gathered}
E_{b}=-6 \frac{Z_{1}^{2} R_{\infty}}{n_{1}^{2}}-\frac{24 K_{1}\left(Z_{1 M}+2 s_{1,2}\right)}{\sqrt{9 a^{2}+25 \sigma^{2}}}+\frac{6 K_{1}}{\sqrt{\sigma^{2}+9 a^{2}}}+\frac{24 K_{1}}{\sqrt{4 \sigma^{2}+9 a^{2}}}+\frac{K_{1} Z_{n A}^{2}}{2 \sigma} \\
E_{b}=-\frac{3 n_{1} h \sqrt{2 K_{1} Z_{1 M}}}{\pi \sqrt{m}\left(\sigma^{2}+a^{2}\right)^{\frac{3}{4}}} \\
E_{b}=-\frac{12 K_{1}\left(Z_{1 M}+2 s_{1,2}\right)}{\sqrt{\sigma^{2}+a^{2}}}+\frac{6 K_{1}}{\sqrt{3} a}+\frac{6 K_{1}}{a}+\frac{3 K_{1}}{2 a}+\frac{K_{1} Z_{n A}^{2}}{2 \sigma}
\end{gathered}
$$

where

$$
Z_{1}=Z_{A}-2 s_{1, A 1}-2 s_{1,2}, \quad Z_{1 M}=Z_{A}-2-2 s_{1,2}
$$




\section{TMPH-2010-0364.revised}

\section{Molecular Physics}

and

$$
s_{1,2}=\frac{\sqrt{2}}{4 \sqrt{1-\frac{\sigma^{2}-\frac{1}{2} a^{2}}{\sigma^{2}+a^{2}}}}
$$

\section{S1.3. Ionic bonds in heteronuclear molecules}

We consider a heteronuclear molecule composed of two fixed different nuclei, denoted by $n_{A}$ and $n_{B}$, two $1 s$ electrons in the vicinity of the $n_{A}$ nucleus, denoted by $e_{A 1}$ and $e_{A 2}$, and two valence electrons which participate to the bond, denoted by $e_{1}$ and $e_{2}$. In Subsection 3.2. we have shown that the curves $C_{1}$ and $C_{2}$ for this molecule are represented in Fig. 3(a) from the paper. These curves are situated in the plane $x y$, the Cartesian coordinates of the nuclei are $n_{A}(0,0,0)$ and $n_{B}\left(\sigma_{1}+\sigma_{2}, 0,0\right)$ and their order numbers are, respectvely, equal to $Z_{A}$ and $Z_{B}$, where $Z_{A}>Z_{B}$. The curve $C_{a}$, where $a=1$ or $a=2$, is composed of two quasilinear ellipses, denoted by $C_{a A}$ and $C_{a B}$, which surround, respectively the nuclei $n_{A}$ and $n_{B}$. We distinguish two phases, the phase $A$, when the electrons are situated on the curves $C_{1 A}$ and $C_{2 A}$, in the vicinity of the nucleus $n_{A}$, and the phase $B$, when the electrons are situated on the curves $C_{1 B}$ and $C_{2 B}$, in the vicinity of the nucleus $n_{B}$. The average positions of the electrons in the phase $A$ are $e_{1}\left[(3 / 4) \sigma_{1},(3 / 4) a, 0\right]$ and $e_{2}\left[(3 / 4) \sigma_{1},-(3 / 4) a, 0\right]$, while in the phase $B$ they are $e_{1}\left[\sigma_{1}+(1 / 4) \sigma_{2},(3 / 4) a, 0\right]$ and $e_{2}\left[\sigma_{1}+(1 / 4) \sigma_{2},-(3 / 4) a, 0\right]$. We note that the curves $C_{1 A}$ and $C_{2 A}$, on one hand, and the curves $C_{1 B}$ and $C_{2 B}$, on the other hand, are very similar to the curves $C_{1}$ and $C_{2}$ in the case of helium atom.

In the case of the heteronuclear diatomic molecules, the order number corresponding to the bond electron $e_{i}$ is denoted by $Z_{i(A)}$ when $e_{i}$ moves in the vicinity of the nucleus $n_{A}$ and by $Z_{i(B)}$ when $e_{i}$ moves in the vicinity of the nucleus $n_{B}$. The screening coefficients between the bond electrons $e_{1}$ and $e_{2}$, which move in the vicinity of the nucleus $n_{A}$, are denoted by $s_{1,2(A)}$ and $s_{2,1(A)}$. The screening coefficients between the electrons $e_{A i}$ and $e_{j}$ when $e_{j}$ moves in the vicinity of the nucleus $n_{A}$ have the same significance, as they do in the case of the homonuclear molecules, and therefore we keep the same notations for them, namely $s_{A i, j}$ and $s_{j, A i}$.

The treatment of this system is almost identical to that presented in Appendix A from the paper, for the simple bond, with the difference that, due to asymmetry, the order numbers are different, for the two phases, as follows

$$
\begin{gathered}
Z_{1(A)}=Z_{A}-2 s_{1, A 1}-s_{1,2(A)}, \quad Z_{1 M(A)}=Z_{A}-2-s_{1,2(A)} \text { and } Z_{n A}=Z_{A}-2 \\
Z_{1(B)}=Z_{B}-s_{1,2(B)}, \quad Z_{1 M(B)}=Z_{B}-s_{1,2(B)} \text { and } Z_{n B}=Z_{B}
\end{gathered}
$$

where

$$
s_{1,2(A)}=\frac{\sqrt{\sigma_{1}^{2}+a^{2}}}{4 a} \text { and } s_{1,2(B)}=\frac{\sqrt{\sigma_{2}^{2}+a^{2}}}{4 a}
$$

Because of this difference between phases, the quantum numbers associated to the motion of the electron $e_{1}$ on curves $C_{1 A}$ and $C_{2 B}$, denoted, respectively, by $n_{1(A)}$ and $n_{1(B)}$, and the corresponding periods, denoted by $\tau_{A}$ and $\tau_{B}$, are different.

Due to the symmetry of the system, we have $Z_{1(A)}=Z_{2(A)}, Z_{1(B)}=Z_{2(B)}$, $Z_{1 M(A)}=Z_{2 M(A)}, Z_{1 M(B)}=Z_{2 M(B)}, n_{1(A)}=n_{2(A)}$ and $n_{1(B)}=n_{2(B)}$. 
The kinetic energy of one electron, corresponding to the points $A_{1}$ or $A_{2}$ is denoted by $T_{m}$. Despite the fact that its value is very small, is it not neglected. The three types of equations from the previous subsection are derived in a similar manner, as follows.

1) The first type of relation. These relations result from the central field approximation, using the quantization condition. The energy of the electron $e_{1}$, when the system is in the phase $A$, is

$$
E_{1}=T_{1}+U_{e_{1} n_{A}}=\frac{E_{b}}{2}-U_{0 A} \text { with } U_{0 A}=U_{e_{1} n_{B}}+\frac{1}{2} U_{n_{A} n_{B}}
$$

Since the $e_{1}$ and $e_{2}$ electrons are situated in the vicinity of the nucleus $n_{A}$, their interaction energy is included in $U_{e_{1} n_{A}}$ and $U_{e_{2} n_{A}}$, by the term $s_{1,2(A)}$.

The quantization relation, applied for the curve $C_{1 A}$, is $\Delta_{C_{1 A}} S_{01}=n_{1(A)} h$. The processing of these relations is identical to the processing of relations (A1), (A3) and (A4) from paper. Thus, we approximate the quantity $U_{0 A}$, with its value, denoted by $U_{0 A m}$, calculated when the electrons are in average positions. In this case, the motions of the electrons reduces to that in a central field. An identical procedure to that presented in Appendix A from the paper leads to the following relation

$$
E_{b}=-2 \frac{Z_{1(A)}^{2} R_{\infty}}{n_{1(A)}^{2}}-\frac{8 K_{1}\left[Z_{1 M(B)}+s_{1,2(B)}\right]}{\sqrt{9 a^{2}+\left(\sigma_{1}+4 \sigma_{2}\right)^{2}}}+\frac{K_{1} Z_{n A} Z_{n B}}{\sigma_{1}+\sigma_{2}}
$$

The same analysis, made for phase $B$, leads to the following relation

$$
E_{b}=-2 \frac{Z_{1(B)}^{2} R_{\infty}}{n_{1(B)}^{2}}-\frac{8 K_{1}\left[Z_{1 M(A)}+s_{1,2(A)}\right]}{\sqrt{9 a^{2}+\left(\sigma_{2}+4 \sigma_{1}\right)^{2}}}+\frac{K_{1} Z_{n A} Z_{n B}}{\sigma_{1}+\sigma_{2}}
$$

2) The second type of relation. These relations result from the virial theorem. We show that this theorem can be written separately for phases $A$ and $B$. We take into account the notations from Fig. 3(a) from the paper and write the following relation for the phase $A: d\left(m \bar{r} \cdot \bar{v}_{1}\right) / d t+d\left(m \bar{r}^{\prime} \cdot \bar{v}_{2}\right) / d t=m \bar{r} \cdot d \bar{v}_{1} / d t+m \bar{r}^{\prime}$. $d \bar{v}_{2} / d t+m v_{1}^{2}+m v_{2}^{2}$. The integration of this relation with respect to time, over the period $\tau_{A}$, considering that $\bar{r} \cdot \bar{v}_{1} \cong 0$ and $\bar{r}^{\prime} \cdot \bar{v}_{2} \cong 0$, respectively, at points $A_{1}$ and $A_{2}$, leads to the following relation:

$$
2 \tilde{T}=-\frac{1}{\tau_{A}} \int_{\tau_{A}}\left(\bar{r} \cdot \bar{F}_{1}+\bar{r}^{\prime} \cdot \bar{F}_{2}\right) d t
$$

where $\bar{F}_{1}$ and $\bar{F}_{2}$ are the forces which act, respectively, on the electrons $e_{1}$ and $e_{2}$. With the aid of their expressions [see Fig. 3(a) from the paper], and taking into account the symmetry of the system and the fact that $\bar{r}=-\sigma_{1} \bar{i}+\bar{r}_{1}=\sigma_{2} \bar{i}+\bar{r}_{1}^{\prime}$ and $\bar{r}^{\prime}=-\sigma_{1} \bar{i}+\bar{r}_{2}=\sigma_{2} \bar{i}+\bar{r}_{2}^{\prime}$, we obtain 


$$
\begin{aligned}
& \bar{r} \cdot \bar{F}_{1}+\bar{r}^{\prime} \cdot \bar{F}_{2}= {\left[-\frac{K_{1} Z_{1(A)}}{r_{1}^{3}} \bar{r}_{1}-\frac{K_{1} Z_{1 M(B)}}{r_{1}^{\prime 3}} \bar{r}_{1}^{\prime}+\frac{K_{1}}{\left|\bar{r}_{1}-\bar{r}_{2}\right|^{3}}\left(\bar{r}_{1}-\bar{r}_{2}\right)\right] \bar{r}+(\mathrm{S} 18) } \\
& {\left[-\frac{K_{1} Z_{1(A)}}{r_{2}^{3}} \bar{r}_{2}-\frac{K_{1} Z_{1 M(B)}}{r_{2}^{\prime 3}} \bar{r}_{2}^{\prime}+\frac{K_{1}}{\left|\bar{r}_{2}-\bar{r}_{1}\right|^{3}}\left(\bar{r}_{2}-\bar{r}_{1}\right)\right] \bar{r}^{\prime}=} \\
& 2 \sigma_{1} \frac{K_{1} Z_{1(A)}}{r_{1}^{2}} \cos \alpha_{1}+2 \sigma_{2} \frac{K_{1} Z_{1 M(B)}}{r_{1}^{\prime 2}} \cos \alpha_{2}+U-\frac{K_{1} Z_{n A} Z_{n B}}{\sigma_{1}+\sigma_{2}}
\end{aligned}
$$

where $U=-2 K_{1} Z_{1(A)} / r_{1}-2 K_{1} Z_{1 M(B)} / r_{1}^{\prime}+K_{1} /\left|\bar{r}_{1}-\bar{r}_{2}\right|+K_{1} Z_{n A} Z_{n B} /\left(\sigma_{1}+\sigma_{2}\right)$. This is the potential energy of the system.

Introducing the above relation in (S17) and supposing that the average forces which act on the two nuclei are zero, we obtain $2 \tilde{T}=-\bar{U}$. Since $E_{b}=\widetilde{U}+\widetilde{T}$, we have:

$$
E_{b}=-\tilde{T}=-\frac{1}{\tau_{A}} \int_{\tau} m v_{1}^{2} d t=-\frac{\Delta_{C_{1 A}} S_{01}}{\tau_{A}}=-\frac{n_{1(A)} h}{\tau_{A}}
$$

In this case $\tau_{A}=2 \int_{0}^{r_{M(A)}} d r_{1} / v_{1}=2 \int_{0}^{r_{M(A)}} d r_{1} / \sqrt{2 T_{1} / m}$ where $T_{1}=T_{m}$ for $r_{1}=r_{M(A)}=\sqrt{\sigma_{1}^{2}+a^{2}}$. Since $T_{m}$ is a very small quantity, we consider again that the domain in the vicinity of the point $A_{1}$ has a big weight in the calculation of the integral. We, thus, have $U_{e_{1} n_{A}} \cong-K_{1} Z_{1 M(A)} / r_{1}$. The calculation of $\tau_{A}$ is performed similarly as in (A9) from paper. Using (S14) it follows that $E_{b} / 2-U_{0}=T_{1}-K_{1} Z_{1 M(A)} / r_{1}=T_{m}-K_{1} Z_{1 M(A)} / r_{M(A)}$, or $T_{1}=$ $K_{1} Z_{1 M(A)} / r_{1}-K_{1} Z_{1 M(A)} / r_{M(A)}+T_{m}$ and, instead of (A9) from paper, we write

$$
\tau_{A}=\sqrt{2 m} \int_{0}^{r_{M(A)}} \frac{d r_{1}}{\sqrt{\frac{K_{1} Z_{1 M(A)}}{r_{1}}-\frac{K_{1} Z_{1 M(A)}}{r_{M(A)}}+T_{m}}}=\pi \sqrt{\frac{m}{2 K_{1} Z_{1 M(A)}}} r_{M(A)}^{\frac{3}{2}} t_{c A}
$$

where $t_{c A}$ is a correction term, given by the relation

$$
t_{c A}=\frac{2}{\pi a_{c A}^{3 / 2}}\left(\arcsin \sqrt{a_{c A}}-\sqrt{a_{c A}} \sqrt{1-a_{c A}}\right)
$$

with

$$
a_{c A}=1-\frac{T_{m} \sqrt{\sigma_{1}^{2}+a^{2}}}{K_{1} Z_{1 M(A)}}
$$

Since $r_{M(A)}=\sqrt{\sigma_{1}^{2}+a^{2}}$, from (S19) and (S20) we obtain

$$
E_{b}=-\frac{n_{1(A)} h \sqrt{2 K_{1} Z_{1 M(A)}}}{\pi \sqrt{m}\left(\sigma_{1}^{2}+a^{2}\right)^{\frac{3}{4}} t_{c A}}
$$

An identical analysis, corresponding to phase $B$, leads to the following relations:

$$
E_{b}=-\frac{n_{1(B)} h \sqrt{2 K_{1} Z_{1 M(B)}}}{\pi \sqrt{m}\left(\sigma_{2}^{2}+a^{2}\right)^{\frac{3}{4}} t_{c B}}
$$


where

$$
t_{c B}=\frac{2}{\pi a_{c B}^{3 / 2}}\left(\arcsin \sqrt{a_{c B}}-\sqrt{a_{c B}} \sqrt{1-a_{c B}}\right)
$$

and

$$
a_{c B}=1-\frac{T_{m} \sqrt{\sigma_{2}^{2}+a^{2}}}{K_{1} Z_{1 M(B)}}
$$

3) The third type of relation. This relation, which is the expression of the energy $E_{b}$, when the electrons are situated, respectively, at points $A_{1}(0, a, 0)$ and $A_{2}(0,-a, 0)$, results directly

$$
\begin{array}{r}
E_{b}=-\frac{2 K_{1}\left[Z_{1 M(A)}+s_{1,2(A)}\right]}{\sqrt{\sigma_{1}^{2}+a^{2}}}-\frac{2 K_{1}\left[Z_{1 M(B)}+s_{1,2(B)}\right]}{\sqrt{\sigma_{2}^{2}+a^{2}}}+\frac{K_{1}}{2 a}+ \\
\frac{K_{1} Z_{n A} Z_{n B}}{\sigma_{1}+\sigma_{2}}+2 T_{m}
\end{array}
$$

\section{S1.4. Covalent bonds in heteronuclear diatomic molecules.}

We consider the same heteronuclear covalent diatomic molecule as that presented in Subsection 3.2 from paper, having $n_{A}>n_{B}$, when the $e_{1}$ and $e_{2}$ electrons are situated in the vicinities of different nuclei, as shown in Fig. 3(b) from paper. The average positions of the electrons are $e_{1}\left[(3 / 4) \sigma_{1},(3 / 4) a, 0\right]$ and $e_{1}\left[\left(\sigma_{1}+(1 / 4) \sigma_{2}\right),-(3 / 4) a, 0\right]$. The curves $C_{1}$ and $C_{2}$ are asymmetrical, similar to those shown in Fig. 3(a) from paper. The treatment of this system is almost identical to that from Appendix A from paper for simple bond, with the difference that the order numbers are different. For the positions of the electrons shown in Fig. 3(b) from paper, we have

$$
\begin{gathered}
Z_{1(A)}=Z_{A}-2 s_{1, A 1}, \quad Z_{1 M(A)}=Z_{A}-2, \quad \text { and } Z_{n A}=Z_{A}-2 \\
Z_{2(B)}=Z_{B}, \quad Z_{2 M(B)}=Z_{B} \text { and } Z_{n B}=Z_{B}
\end{gathered}
$$

The quantum numbers associated to the electrons $e_{1}$ and $e_{2}$, for the positions shown in Fig. 3(b) from paper, are respectively, $n_{1(A)}$ and $n_{2(B)}$. Taking into account the symmetry of the curves $C_{1}$ and $C_{2}$, we have $Z_{1(A)}=Z_{2(A)}, Z_{1(B)}=Z_{2(B)}$, $Z_{1 M(A)}=Z_{2 M(A)}, Z_{1 M(B)}=Z_{2 M(B)}, n_{1(A)}=n_{2(A)}$ and $n_{1(B)}=n_{2(B)}$.

Both electrons move alternatively in the fields of the two nuclei, on the curves $C_{1 A}, C_{1 B}$ and $C_{2 A}, C_{2 B}$. We assume that the kinetic energies of the bond electrons in the points $A_{1}$ and $A_{2}$ are neglected, as in Appendix A from paper, and that the motion of the electrons is periodical. The periodicity leads to the following relation

$$
\tau_{A}=\tau_{B}
$$

In the case of the ionic bond, the electrons move together on helium type trajectories in the field of the same nucleus, and due to the the nonsymmetry of the $C_{a}$ trajectory, in the majority of the time, the electrons are in the vicinity of the nucleus whose order number is smaller. Unlike the ionic bond, in the present case, the electrons move alternatively in the fields of different nuclei, the two periods of 


\section{Molecular Physics}

motion, $\tau_{A}$ and $\tau_{B}$, are equal and their charge is disposed in the vicinities of both nuclei, from here resulting the covalent character of the bond.

The three types of equations from the previous subsection, result, similarly as in Appendix A from paper, as follows.

1) The first type of relation. This relation results from the central field approximation, using the quantization condition. In this case the expression of the energy $E_{b}$ can be written:

$$
E_{b}=E_{1}+E_{2}+U_{01}+U_{02} \text { with } E_{1}=T_{1}+U_{e_{1} n_{A}} \text { and } E_{2}=T_{2}+U_{e_{2} n_{B}}
$$

where

$$
U_{01}=U_{e_{1} n_{B}}+\frac{1}{2}\left(U_{e_{1} e_{2}}+U_{n_{A} n_{B}}\right) \text { and } U_{02}=U_{e_{2} n_{A}}+\frac{1}{2}\left(U_{e_{1} e_{2}}+U_{n_{A} n_{B}}\right)
$$

We consider that the motions of the $e_{1}$ and $e_{2}$ electrons are approximated by motions in the in central fields of the nuclei $n_{a}$ and $n_{B}$. In this case $E_{1}$ and $E_{2}$ are constants and the quantities $U_{01}$ and $U_{02}$ are calculated for the average positions of the electrons. We apply quantization relations $\Delta_{C_{1 A}} S_{01}=n_{1(A)} h$ and $\Delta_{C_{2 B}} S_{02}=$ $n_{2(B)} h$, respectively, for the curves $C_{1 A}$ and $C_{2 B}$. We obtain $E_{1}=-R_{\infty} Z_{1(A)}^{2} / n_{1(A)}^{2}$ and $E_{2}=-R_{\infty} Z_{2(B)}^{2} / n_{2(B)}^{2}$. Taking into account (S31) and (S32), we obtain:

$$
\begin{gathered}
E_{b}=-\frac{R_{\infty} Z_{1(A)}^{2}}{n_{1(A)}^{2}}-\frac{R_{\infty} Z_{2(B)}^{2}}{n_{2(B)}^{2}}-\frac{4 K_{1} Z_{1 M(B)}}{\sqrt{9 a^{2}+\left(\sigma_{1}+4 \sigma_{2}\right)^{2}}}- \\
\frac{4 K_{1} Z_{1 M(A)}}{\sqrt{9 a^{2}+\left(\sigma_{2}+4 \sigma_{1}\right)^{2}}}+\frac{4 K_{1}}{\sqrt{36 a^{2}+\left(\sigma_{1}+\sigma_{2}\right)^{2}}}+\frac{Z_{n A} Z_{n B}}{\sigma_{1}+\sigma_{2}}
\end{gathered}
$$

2) The second type of relation. The virial theorem, written for the curve $C_{1}$, and taking into account the symmetry of the curves $C_{1}$ and $C_{2}$, is

$$
\begin{aligned}
E_{b}=-\frac{1}{\tau_{A}} \int_{\tau_{A}} \frac{m v_{1}^{2}}{2} d t-\frac{1}{\tau_{B}} \int_{\tau_{B}} \frac{m v_{2}^{2}}{2} d t= & -\frac{\Delta S_{01}}{2 \tau_{A}}-\frac{\Delta S_{02}}{2 \tau_{B}}= \\
& -\frac{n_{1(A)} h}{2 \tau_{A}}-\frac{n_{2(B)} h}{2 \tau_{B}}
\end{aligned}
$$

which, in virtue of equation (S30), can be written:

$$
2 \tau_{A} E_{b}=2 \tau_{B} E_{b}=-\left[n_{1(A)}+n_{1(B)}\right] h
$$

A calculation similar to that presented in Appendix A from paper, which takes into account (S31), leads to the following relation:

$$
\begin{array}{r}
\tau_{A}=\tau_{B}=\pi \sqrt{\frac{m}{2 K_{1} Z_{1 M(A)}}}\left(\sigma_{1}^{2}+a^{2}\right)^{\frac{3}{4}}=\pi \sqrt{\frac{m}{2 K_{1} Z_{1 M(B)}}}\left(\sigma_{2}^{2}+a^{2}\right)^{\frac{3}{4}}= \\
-\frac{\left[n_{1(A)}+n_{1(B)}\right] h}{2 E_{b}}
\end{array}
$$

3) The third type of relation. This relation, which is the expression of the energy $E_{B}$, when the electrons are situated, respectively, at points $A_{1}(0, a, 0)$ and 


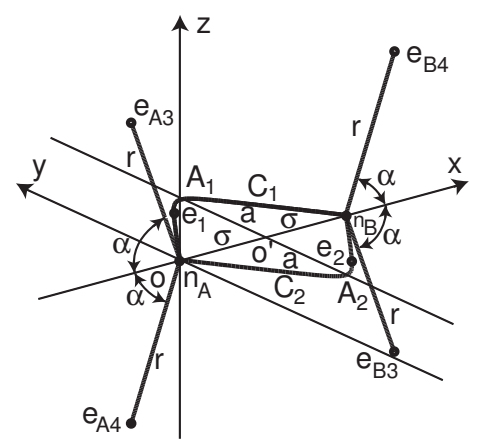

Figure S1. Structure of $B_{2}$ molecule.

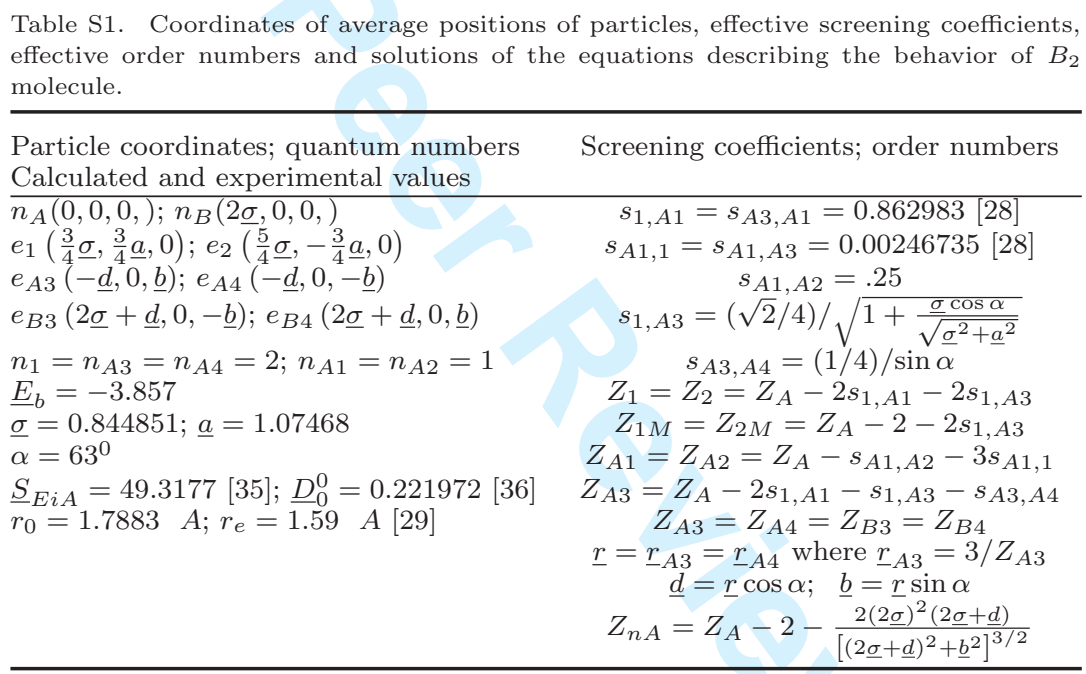

$A_{2}(0,-a, 0)$, results directly

$$
E_{b}=-\frac{2 K_{1} Z_{1 M(A)}}{\sqrt{\sigma_{1}^{2}+a^{2}}}-\frac{2 K_{1} Z_{1 M(B)}}{\sqrt{\sigma_{2}^{2}+a^{2}}}+\frac{K_{1}}{2 a}+\frac{K_{1} Z_{n A} Z_{n B}}{\sigma_{1}+\sigma_{2}}
$$

S2. Details of the calculations for $\mathrm{B}_{2}, \mathrm{C}_{2}, \mathrm{LiH}, \mathrm{BeH}, \mathrm{BH}$ and $\mathrm{CH}$ molecules.

\section{S2.1. $B_{2}$ molecule}

The structure of this molecule is shown in Fig. S1. This molecule contains four electrons, denoted by $e_{A 3}, e_{A 4}, e_{B 3}$ and $e_{B 4}$ which do not participate to the bond. All the data necessary in calculations are given in Table S1.

The geometrical structure of the molecule results from the system (34)-(36) from paper, while the normalized value of the total energy is calculated with the aid of equation (41) from paper, and can be written, taking into account the symmetry of the system (namely $\underline{E}_{1}=\underline{E}_{2}, \underline{E}_{A 3}=\underline{E}_{A 4}=\underline{E}_{B 3}=\underline{E}_{B 4}, \underline{E}_{A 1}=\underline{E}_{A 2}=\underline{E}_{B 1}=$ $\underline{E}_{B 2}, \underline{U}_{e_{1} n_{B}}=\underline{U}_{e_{2} n_{A}}, \underline{U}_{e_{A 3} e_{2}}=\underline{U}_{e_{A 4} e_{2}}=\underline{U}_{e_{B 3} e_{1}}=\underline{U}_{e_{B 4} e_{1}}, \underline{U}_{e_{A 3} n_{B}}=\underline{U}_{e_{A 4} n_{B}}=$ 


\section{S2.2. $C_{2}$ molecule}

We analize the $C_{2}$ molecule in three cases: when the bond is simple, double and triple. We calculate the total energy and choose the case having the lowest total energy. We consider that this case corresponds to the real $C_{2}$ molecule.

1) $C_{2}$ molecule with simple bond. The structure of this molecule is shown in Fig. S2(a). This molecule contains six electrons, denoted by $e_{A 3}, e_{A 4}, e_{A 5}, e_{B 3}$, $e_{B 4}$, and $e_{B 5}$ which do not participate to the bond. The coordinates of these electrons are as follows: $e_{A 3}(-\underline{d}, 0, \underline{b}), e_{A 4}\left(-\underline{d}, \frac{\sqrt{3}}{2} \underline{b},-\frac{1}{2} \underline{b}\right), e_{A 5}\left(-\underline{d},-\frac{\sqrt{3}}{2} \underline{b},-\frac{1}{2} \underline{b}\right)$, $e_{B 3}(2 \underline{\sigma}+\underline{d}, 0,-\underline{b}), e_{B 4}\left(2 \underline{\sigma}+\underline{d},-\frac{\sqrt{3}}{2} \underline{b}, \frac{1}{2} \underline{b}\right)$ and $e_{B 5}\left(2 \underline{\sigma}+\underline{d}, \frac{\sqrt{3}}{2} \underline{b}, \frac{1}{2} \underline{b}\right)$. A calculation identical to that for the $B_{2}$ molecule leads to the following values: $\underline{E}_{b}=$ $-5.69164, \underline{\sigma}=0.751248, \underline{a}=0.857921, r_{0}=1.59018 A$ and $\underline{E}=-150.365$.

2) $C_{2}$ molecule with double bond. The structure of this molecule is shown in Fig. S2(b). This molecule contains four electrons, denoted by $e_{A 3}, e_{A 4}, e_{B 3}$ and $e_{B 4}$, which do not participate to the bond. The geometrical structure of the molecule results from the system (S3)-(S5), which can be written in normalized form, as follows:

$$
\begin{gathered}
\underline{E}_{b}=-4 \frac{Z_{1}^{2}}{n_{1}^{2}}-\frac{16\left(Z_{1 M}+s_{1,2}\right)}{\sqrt{9 \underline{a}^{2}+25 \underline{\sigma}^{2}}}+\frac{8}{\sqrt{\underline{\sigma}^{2}+4.5 \underline{a}^{2}}}+\frac{Z_{n A}^{2}}{2 \underline{\sigma}} \\
\underline{E}_{b}=-\frac{4 n_{1} \sqrt{Z_{1 M}}}{\left(\underline{a}^{2}+\underline{\sigma}^{2}\right)^{3 / 4}}
\end{gathered}
$$




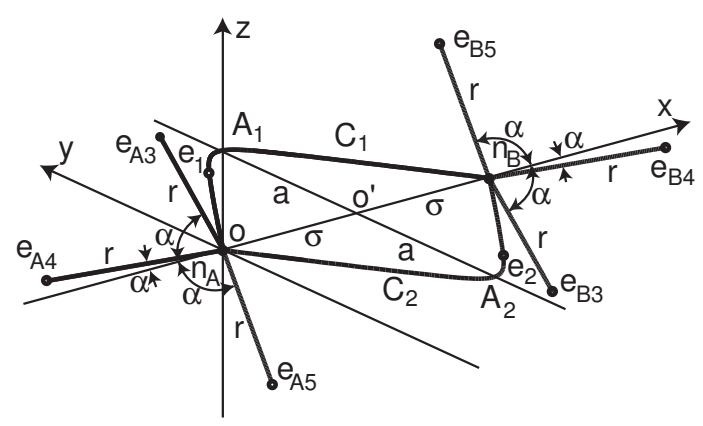

(a)

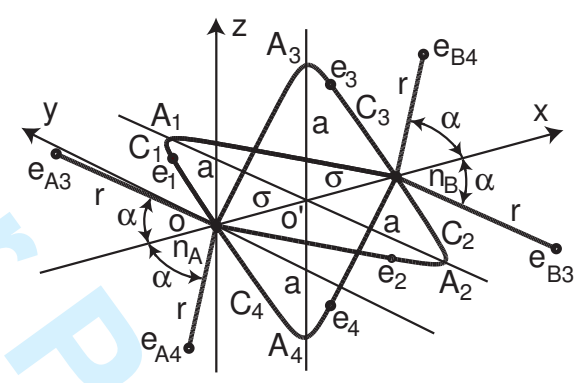

(b)

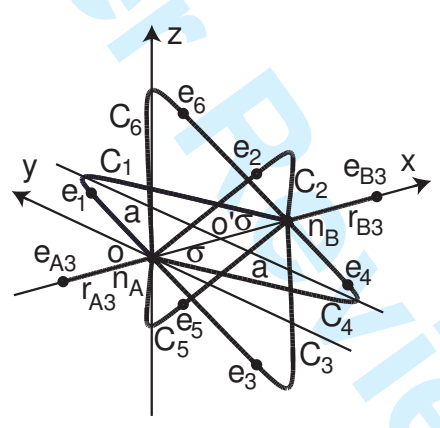

(c)

Figure S2. Structures of (a) simple, (b) double and (c) triple bonds in $C_{2}$ molecule.

$$
\underline{E}_{b}=-\frac{8\left(Z_{1 M}+s_{1,2}\right)}{\sqrt{\underline{\sigma}^{2}+\underline{a}^{2}}}+\frac{1}{\underline{a}}+\frac{2 \sqrt{2}}{\underline{a}}+\frac{Z_{n A}^{2}}{2 \underline{\sigma}}
$$

All the data necessary for calculations, together with the experimental data and the results of the calculations are given in Table S2. The angle $\alpha$ corresponds to the minimum value of the total energy. The normalized value of the total energy is calculated with the aid of equation (41) from paper. Taking into account the symmetry of the system (namely $\underline{E}_{1}=\underline{E}_{2}=\underline{E}_{3}=\underline{E}_{4}, \underline{E}_{A 3}=\underline{E}_{A 4}=\underline{E}_{B 3}=\underline{E}_{B 4}$, $\underline{E}_{A 1}=\underline{E}_{A 2}=\underline{E}_{B 1}=\underline{E}_{B 2}, \underline{U}_{e_{1} e_{3}}=\underline{U}_{e_{1} e_{4}}=\underline{U}_{e_{2} e_{3}}=\underline{U}_{e_{2} e_{4}}, \underline{U}_{e_{1} n_{B}}=\underline{U}_{e_{2} n_{B}}=$ $\underline{U}_{e_{3} n_{A}}=\underline{U}_{e_{4} n_{A}}, \underline{U}_{e_{A 3} e_{3}}=\underline{U}_{e_{A 4} e_{4}}=\underline{U}_{e_{B 3} e_{2}}=\underline{U}_{e_{B 4} e_{1}}, \underline{U}_{e_{A 3} e_{4}}=\underline{U}_{e_{A 4} e_{3}}=\underline{U}_{e_{B 3} e_{1}}=$ $\underline{U}_{e_{B 4} e_{2}}, \underline{U}_{e_{A 3} n_{B}}=\underline{U}_{e_{A 4} n_{B}}=\underline{U}_{e_{B 3} n_{A}}=\underline{U}_{e_{B 4} n_{A}}, \underline{U}_{e_{A 3} e_{B 3}}=\underline{U}_{e_{A 4} e_{B 4}}$ and $\underline{U}_{e_{A 3} e_{B 4}}=$ 
Table S2. Coordinates of average positions of particles, effective screening coefficients, effective order numbers and solutions of the equations describing the behavior of $C_{2}$ molecule in the case of double bond.

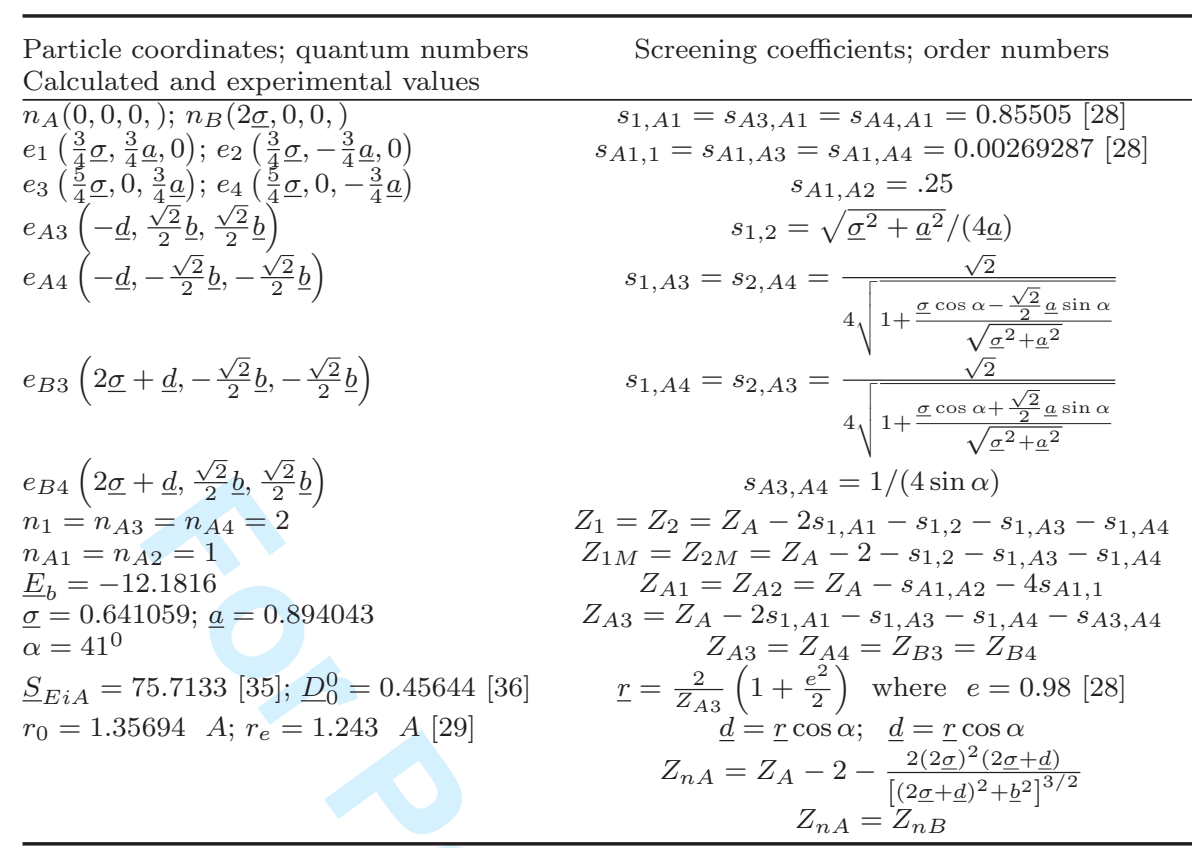

$\left.\underline{U}_{e_{A 4} e_{B 3}}\right)$, this relation can be written for $C_{2}$ molecule with double bond, as follows:

$$
\begin{gathered}
\underline{E}=4 \underline{E}_{1}+4 \underline{E}_{A 3}+4 \underline{E}_{A 1}+4 \underline{U}_{e_{1} e_{3}}+4 \underline{U}_{e_{1} n_{B}}+4 \underline{U}_{e_{A 3} n_{B}}+2 \underline{U}_{e_{A 3} e_{B 3}}+ \\
2 \underline{U}_{e_{A 3} e_{B 4}}+4 \underline{U}_{e_{A 3} e_{3}}+4 \underline{U}_{e_{A 3} e_{4}}+\underline{U}_{n_{A} n_{B}}+2 \underline{E}_{m 1 s}=-4 \frac{Z_{1}^{2}}{n_{1}^{2}}-4 \frac{Z_{A 3}^{2}}{n_{A 3}^{2}}- \\
4 \frac{Z_{A 1}^{2}}{n_{A 1}^{2}}+\frac{4}{\sqrt{\left(\frac{1}{2} \underline{\sigma}\right)^{2}+2\left(\frac{3}{4} \underline{a}\right)^{2}}}-\frac{4\left(Z_{A}-2\right)}{\sqrt{\left(\frac{5}{4} \underline{\sigma}\right)^{2}+\left(\frac{3}{4} \underline{a}\right)^{2}}}-\frac{4\left(Z_{A}-2\right)}{\sqrt{(\underline{d}+2 \underline{\sigma})^{2}+\underline{b}^{2}}}+ \\
\frac{1}{\sqrt{(\underline{d}+\underline{\sigma})^{2}+\underline{b}^{2}}}+\frac{1}{\underline{d}+\underline{\sigma}}+\frac{4}{\sqrt{\left(\underline{d}+\frac{5}{4} \underline{\sigma}\right)^{2}+\left(\frac{\sqrt{2}}{2} \underline{b}\right)^{2}+\left(\frac{3}{4} \underline{a}-\frac{\sqrt{2}}{2} \underline{b}\right)^{2}}}+ \\
\frac{4}{\sqrt{\left(\underline{d}+\frac{5}{4} \underline{\sigma}\right)^{2}+\left(\frac{\sqrt{2}}{2} \underline{b}\right)^{2}+\left(\frac{3}{4} \underline{a}+\frac{\sqrt{2}}{2} \underline{b}\right)^{2}}}+\frac{\left(Z_{A}-2\right)^{2}}{2 \underline{\sigma}}+2 \frac{Z_{A 1}^{3 / 2}}{8 n_{A 1}^{3}}
\end{gathered}
$$

From this relation, we obtain $\underline{E}=-151.148$.

3) $C_{2}$ molecule with triple bond. The structure of this molecule is shown in Fig. S2(c). This molecule contains two electrons, denoted by $e_{A 3}$ and $e_{B 3}$, which do not participate to the bond. The geometrical structure of the molecule results from the system (S6)-(S8), which can be written in normalized form, as follows:

$$
\begin{gathered}
\underline{E}_{b}=-6 \frac{Z_{1}^{2}}{n_{1}^{2}}-\frac{24\left(Z_{1 M}+2 s_{1,2}\right)}{\sqrt{9 \underline{a}^{2}+25 \underline{\sigma}^{2}}}+\frac{6}{\sqrt{\underline{\sigma}^{2}+9 \underline{a}^{2}}}+\frac{24}{\sqrt{4 \underline{\sigma}^{2}+9 \underline{a}^{2}}}+\frac{Z_{n A}^{2}}{2 \underline{\sigma}} \\
\underline{E}_{b}=-\frac{6 n_{1} \sqrt{Z_{1 M}}}{\left(\underline{a}^{2}+\underline{\sigma}^{2}\right)^{3 / 4}}
\end{gathered}
$$




\section{Molecular Physics}

Molecular Physics

Table S3. Coordinates of average positions of particles, effective screening coefficients, effective order numbers and solutions of the equations describing the behavior of $C_{2}$ molecule in the case of triple bond.

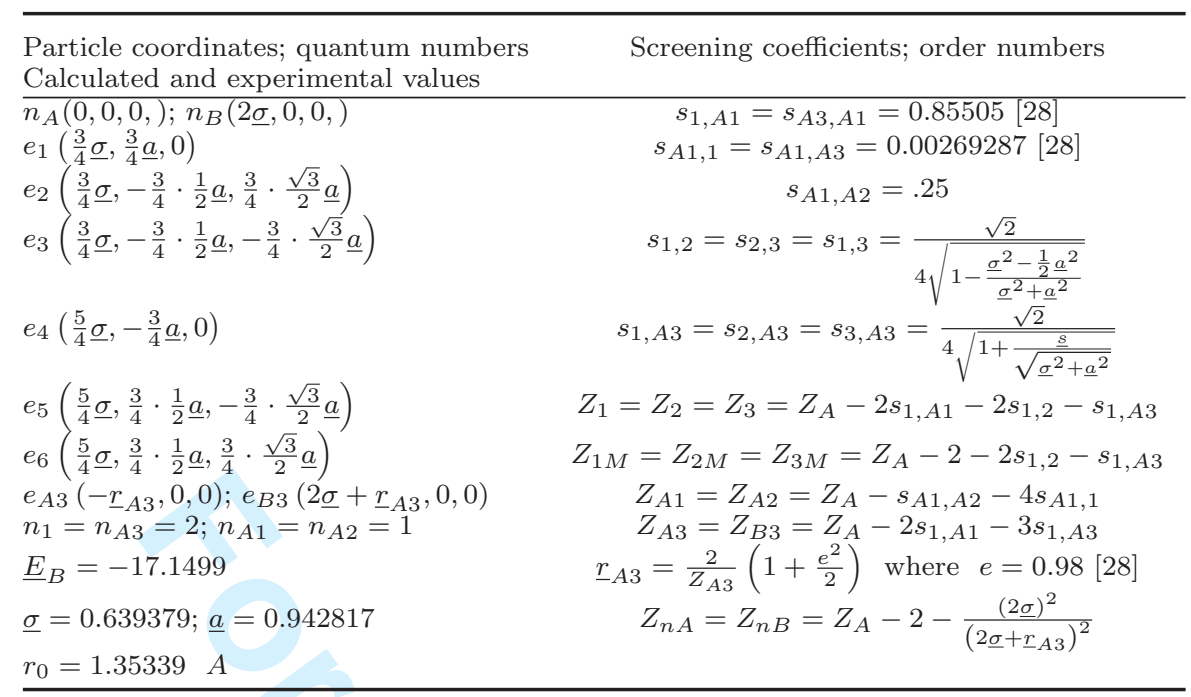

$$
\underline{E}_{b}=-\frac{12\left(Z_{1 M}+2 s_{1,2}\right)}{\sqrt{\underline{\sigma}^{2}+\underline{a}^{2}}}+\frac{6}{\sqrt{3} \underline{a}}+\frac{6}{\underline{a}}+\frac{3}{2 \underline{a}}+\frac{Z_{n A}^{2}}{2 \underline{\sigma}}
$$

All the experimental and theoretical data, excepting the total energy, are given in Table S3. Taking into account the symmetry of the system (namely $\underline{E}_{1}=\underline{E}_{2}=$ $\ldots=\underline{E}_{6}, \underline{E}_{A 3}=\underline{E}_{B 3}, \underline{E}_{A 1}=\underline{E}_{A 2}=\underline{E}_{B 1}=\underline{E}_{B 2}, \underline{U}_{e_{1} e_{4}}=\underline{U}_{e_{2} e_{5}}=\underline{U}_{e_{3} e_{6}}$, $\underline{U}_{e_{1} e_{5}}=\underline{U}_{e_{5} e_{3}}=\underline{U}_{e_{3} e_{4}}=\underline{U}_{e_{4} e_{2}}=\underline{U}_{e_{2} e_{6}}=\underline{U}_{e_{6} e_{1}}, \underline{U}_{e_{A 3} n_{B}}=\underline{U}_{e_{B 3} n_{A}}, \underline{U}_{e_{i} n_{B}}=\underline{U}_{e_{j} n_{A}}$, $\underline{U}_{e_{A 3} e_{j}}=\underline{U}_{e_{B 3} e_{i}}$, where $i=1,2,3$ and $\left.j=3,4,5\right)$, the total energy results from the following relation:

$$
\begin{gathered}
\underline{E}=6 \underline{E}_{1}+2 \underline{E}_{A 3}+4 \underline{E}_{A 1}+3 \underline{U}_{e_{1} e_{4}}+6 \underline{U}_{e_{1} e_{5}}+6 \underline{U}_{e_{1} n_{B}}+6 \underline{U}_{e_{A 3} e_{4}}+(\mathrm{S} 46) \\
2 \underline{U}_{e_{A 3} n_{B}}+\underline{U}_{e_{A 3} e_{B 3}}+\underline{U}_{n_{A} n_{B}}+2 \underline{E}_{m 1 s}=-6 \frac{Z_{1}^{2}}{n_{1}^{2}}-2 \frac{Z_{A 3}^{2}}{n_{A 3}^{2}}-4 \frac{Z_{A 1}^{2}}{n_{A 1}^{2}}+ \\
\frac{3}{\sqrt{\left(\frac{1}{2} \underline{\sigma}\right)^{2}+\left(\frac{3}{2} \underline{a}\right)^{2}}}+\frac{6}{\sqrt{\left(\frac{1}{2} \underline{\sigma}\right)^{2}+\left(\frac{3}{4} \underline{a}\right)^{2}}}-\frac{6\left(Z_{A}-2\right)}{\sqrt{\left(\frac{5}{4} \underline{\sigma}\right)^{2}+\left(\frac{3}{4} a\right)^{2}}}+ \\
\frac{6}{\sqrt{\left(\underline{r}_{A 3}+\frac{5}{4} \underline{\sigma}\right)^{2}+\left(\frac{3}{4} \underline{a}\right)^{2}}}-\frac{2\left(Z_{A}-2\right)}{\underline{r}_{A 3}+2 \underline{\sigma}}+\frac{1}{2 \underline{r}_{A 3}+2 \underline{\sigma}}+\frac{\left(Z_{A}-2\right)^{2}}{2 \underline{\sigma}}+2 \frac{Z_{A 1}^{3 / 2}}{8 n_{A 1}^{3}}
\end{gathered}
$$

From this relation, we obtain $\underline{E}=-150.923$.

If we compare the three above values of the total energy, we will see that the energy corresponding to the double bond is minimum, resulting that the real bond of the $C_{2}$ molecule is double. This result is identical to that obtained by pure quantum evaluations (see, for example pag. 97 of Ref. [31]). In Table 1 from paper it is shown the value of $\underline{E}$ corresponding to the $C_{2}$ molecule with double bond, together with $\underline{E}_{H F}$ and $\underline{E}_{e x p}$. The analysis of Table 1 from paper shows that the relative errors corresponding to $\underline{E}$ and $\underline{E}_{H F}$ are, respectively, equal to 0.005 and 0.007 .

A comparison between, on one hand, the symmetry properties of the systems composed of the $C$ atoms and the electrons which do not participate to the bond [see Figs. S2(a), S2(b) and S2(c)], and, on the other hand, the experimental sym- 
metry properties of the ethane, ethylene and acetylene molecules, namely $\mathrm{C}_{2} \mathrm{H}_{6}$, $\mathrm{C}_{2} \mathrm{H}_{4}$ and $\mathrm{C}_{2} \mathrm{H}_{2}$ (see Ref. [29]), shows that these properties are identical. For example, all these structures have a center of symmetry, the configurations of $C_{2} H_{4}$ and of $n_{A}, n_{B},, e_{A 3}, e_{A 4}, e_{B 3}$ and $e_{B 4}$, in the case of double bond, are plane, or the configurations of $C_{2} H_{2}$ and of $n_{A}, n_{B},, e_{A 3}$ and $e_{B 3}$, in the case of triple bond, are linear. This explains why adding 6,4 and, respectively, 2 hydrogen atoms to the structures of $C_{2}$ with simple, double and triple bonds leads to the formation of the $\mathrm{C}_{2} \mathrm{H}_{6}, \mathrm{C}_{2} \mathrm{H}_{4}$ and, respectively, $\mathrm{C}_{2} \mathrm{H}_{2}$ molecules. Also, our calculations explain the property that the length of the bond decreases with the increasing of the bond order.

\section{S2.3. LiH molecule}

We analyze now the following molecules: $\mathrm{LiH}, \mathrm{BeH}, \mathrm{BH}$ and $\mathrm{CH}$. The model of ionic bond is in agreement with the experimental data for $\mathrm{LiH}, \mathrm{BeH}$, while the model of covalent bond is in agreement with the experimental data only for $B H$ and $C H$. For example, if we apply the covalent model in the case of $L i H$, it will lead to a symmetrical molecule, for which $\sigma_{1}=\sigma_{2}$, which is in strong disagreement with the fact that this molecule has a big electrical dipole moment.

The structure of the $\mathrm{LiH}$ molecule molecule is shown in Fig. 3(a) from paper. This molecule contains only valence electrons that participate to the bond. The geometrical structure of the molecule results from the system (S15), (S16), (S23), (S24) and (S27), which can be written in normalized form as follows:

$$
\begin{aligned}
& \underline{E}_{b}=-2 \frac{Z_{1(A)}^{2}}{n_{1(A)}^{2}}-\frac{8\left[Z_{1 M(B)}+s_{1,2(B)}\right]}{\sqrt{9 \underline{a}^{2}+\left(\underline{\sigma}_{1}+4 \underline{\sigma}_{2}\right)^{2}}}+\frac{Z_{n A} Z_{n B}}{\underline{\sigma}_{1}+\underline{\sigma_{2}}} \\
& \underline{E}_{b}=-2 \frac{Z_{1(B)}^{2}}{n_{1(B)}^{2}}-\frac{8\left[Z_{1 M(A)}+s_{1,2(A)}\right]}{\sqrt{9 \underline{a}^{2}+\left(\underline{\sigma}_{2}+4 \underline{\sigma}_{1}\right)^{2}}}+\frac{Z_{n A} Z_{n B}}{\underline{\sigma}_{1}+\underline{\sigma}_{2}} \\
& \underline{E}_{b}=-\frac{2 n_{1(A)} \sqrt{Z_{1 M(A)}}}{\left(\underline{\sigma}_{1}^{2}+\underline{a}^{2}\right)^{\frac{3}{4}} t_{c A}} \\
& \underline{E}_{b}=-\frac{2 n_{1(B)} \sqrt{Z_{1 M(B)}}}{\left(\underline{\sigma}_{2}^{2}+\underline{a}^{2}\right)^{\frac{3}{4}} t_{c B}}
\end{aligned}
$$

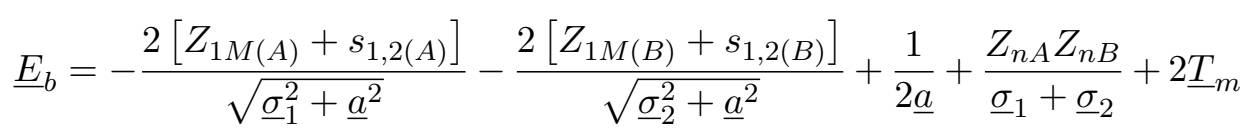

where

$$
t_{c A}=\frac{2}{\pi a_{c A}^{3 / 2}}\left(\arcsin \sqrt{a_{c A}}-\sqrt{a_{c A}} \sqrt{1-a_{c A}}\right)
$$




\section{Molecular Physics}

Molecular Physics

Table S4. Coordinates of average positions of particles, effective screening coefficients, effective order numbers and solutions of the equations describing the behavior of LiH molecule.

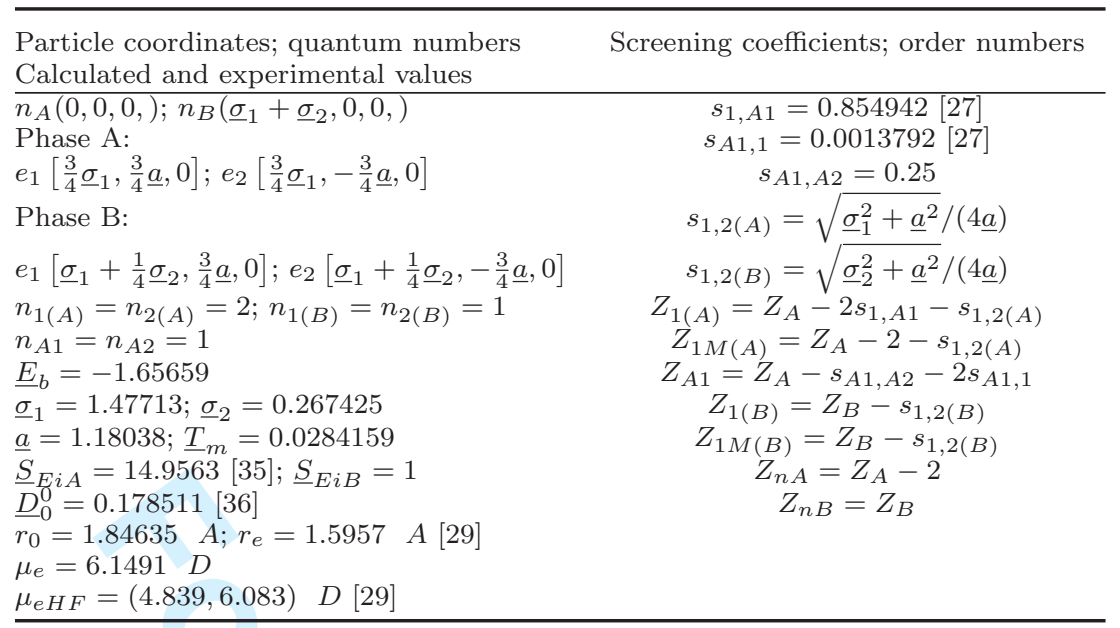

with

$$
a_{c A}=1-\frac{\underline{T}_{m} \sqrt{{\underline{\sigma_{1}^{2}}+\underline{a}^{2}}^{2}}}{Z_{1 M(A)}}
$$

and

$$
t_{c B}=\frac{2}{\pi a_{c B}^{3 / 2}}\left(\arcsin \sqrt{a_{c B}}-\sqrt{a_{c B}} \sqrt{1-a_{c B}}\right)
$$

with

$$
a_{c B}=1-\frac{\underline{T}_{m} \sqrt{{\underline{\sigma_{2}^{2}}+\underline{a}^{2}}^{2}}}{Z_{1 M(B)}}
$$

The system (S47)-(S51) has 5 equations with 5 unknowns, which are $\underline{E}_{b}, \underline{\sigma}_{1}, \underline{\sigma}_{2}, \underline{a}$ and $\underline{T}_{m}$.

We follow again the algorithm presented in Section 4 from paper and calculate the screening coefficients, order numbers and solve the system (S47)-(S51). All these values are given in Table S4. The screening coefficients between the $1 s$ electrons and the $e_{1}$ and $e_{2}$ electrons, namely $s_{1, A 1}$ and $s_{A 1,1}$, when the system is in the phase $A$ are identical, respectively to $s_{31 e}$ and $s_{13 e}$, which have been calculated in Ref. [27] for the $1 s^{2} 2 s$ state of lithium atom. The coefficients $s_{A 1, A 2}, s_{1,2(A)}$ and $s_{1,2(B)}$ are calculated with equation (9). Since $2 s$ and $1 s$ valence electrons participate to the bond, respectively, in phases $A$ and $B$, we have $n_{1(A)}=n_{1(B)}=2$ and $n_{2(A)}=n_{2(B)}=1$.

Since in this case $E_{b}$ has the significance of the total energy from which the energies of the $1 s$ electrons are subtracted, we have:

$$
\underline{E}=\underline{E}_{b}+2 \underline{E}_{A 1}+\underline{E}_{m 1 s}=\underline{E}_{b}-2 \frac{Z_{A 1}^{2}}{n_{A 1}^{2}}+\frac{Z_{A 1}^{3 / 2}}{8 n_{A 1}^{3}}
$$

The normalized value of the total energy, together with the best two values of $\underline{E}_{H F}$, 
obtained from Ref. [29], and the experimental value, $\underline{E}_{\text {exp }}$, given by

$$
\underline{E}_{e x p}=-\underline{S}_{E i A}-\underline{S}_{E i B}-\underline{D}_{0}^{0}
$$

are presented in Table 1 of the paper. The values of $S_{E i A}$ and $S_{E i B}$, which are the sum of the ionization energies of the two atoms, are taken from Ref. [35], while $D_{0}^{0}$ is taken from Ref. [36]. The analysis of Table 1 from paper shows that the relative errors corresponding to $\underline{E}$ and $\underline{E}_{H F}$ are, respectively, equal to 0.003 and 0.010 .

In this case we have

$$
r_{0}=\sigma_{1}+\sigma_{2}
$$

The values of $r_{0}$ and $r_{e}$, which is taken from Ref. [29], are given in angstroms in Table S4.

The electric dipole moment of the molecule is denoted by $\bar{\mu}_{e}$. It is calculated when the electrons are situated at the points $A_{1}$ and $A_{2}$, which are the average positions on the molecular $C_{a}$ curves taking into account the convention that the positive sense of the vector $\bar{\mu}_{e}$ is from the negative charge to the positive charge. In this case, the sign convention is the same as that from Ref. [29]. The expression of $\mu_{e}$ in the case of the $\mathrm{LiH}$ molecule, when the result is given in debyes (D), is

$$
\mu_{e}=-5.08315\left[-2 \underline{\sigma}_{1}+Z_{B}\left(\underline{\sigma}_{1}+\underline{\sigma}_{2}\right)\right]
$$

The theoretical value, calculated with equation (S59), and the experimental value, taken from CCCBDB [29], are equal, respectively, to $6.1491 \mathrm{D}$ and 5.88 D. The CCCBDB does not contain experimental values of the electric dipole moment in the cases of the molecules $\mathrm{BeH}, \mathrm{BH}$ and $\mathrm{CH}$. In Table S4 we compare our theoretical values with the theoretical values of the electric dipole moment, calculated using the Hartree-Fock method, which are taken from Ref. [29]. The analysis of the data from Ref. [29] shows that the dispersion of the theoretical values of the electric dipole moments, in the case of a given system, is very high. For this reason, in Table S4 we give the domain of the theoretical values of the dipole moments, taken from Ref. [29], and computed using the Hartree-Fock method. These values are denoted by $\mu_{e H F}$.

\section{S2.4. BeH molecule}

The structure of this molecule is shown in Fig. S3(a). This molecule contains one electron, denoted by $e_{A 3}$ which do not participate to the bond. The distance $r_{A 3}$ varies slightly when the bond electrons move in the phases $A$ and $B$. Since the bond electrons are in the majority of the time far from nucleus, we consider an average value, approximately equal to that in the case of the isolated atom, which is given by the relation $\underline{\widetilde{r}}_{A 3}=3 /\left(Z_{A}-2 s_{A 3, A 1}\right)$. In this case the value of $E_{b}$ is slightly different in the two phases, being equal, respectively, to $E_{b 1}$ and $E_{b 2}$, in the phases $A$ and $B$. The system (S47)-(S51) becomes

$$
\underline{E}_{b 1}=-2 \frac{Z_{1(A)}^{2}}{n_{1(A)}^{2}}-\frac{8\left[Z_{1 M(B)}+s_{1,2(B)}\right]}{\sqrt{9 \underline{a}^{2}+\left(\underline{\sigma}_{1}+4 \underline{\sigma}_{2}\right)^{2}}}+\frac{Z_{n A} Z_{n B}}{\underline{\sigma}_{1}+\underline{\sigma_{2}}}
$$


1

3

4

5

6

7

8

9

10

11

12

13

14

15

16

17

18

19

20

21

22

23

24

25

26

27

28

29

30

31

32

33

34

35

36

37

38

39

40

41

42

43

44

45

46

47

48

49

50

51

52

53

54

55

56

57

58

59

60

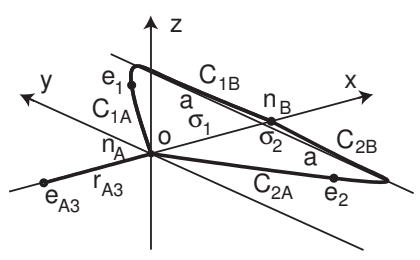

(a)

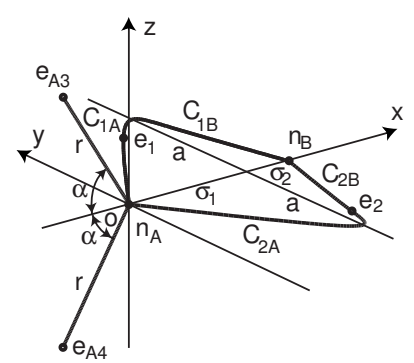

(b)

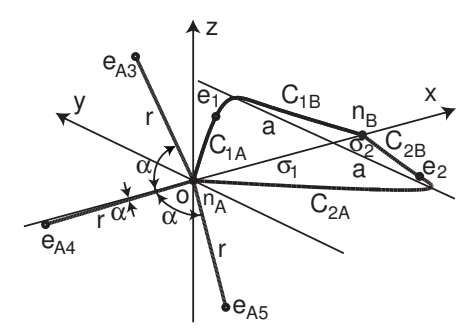

(c)

Figure S3. Structures of (a) BeH, (b) $B H$ and (c) $C H$ molecules.

$$
\underline{E}_{b 2}=-2 \frac{Z_{1(B)}^{2}}{n_{1(B)}^{2}}-\frac{8\left[Z_{1 M(A)}+s_{1,2(A)}\right]}{\sqrt{9 \underline{a}^{2}+\left(\underline{\sigma}_{2}+4 \underline{\sigma}_{1}\right)^{2}}}+\frac{Z_{n A} Z_{n B}}{\underline{\sigma}_{1}+\underline{\sigma}_{2}}
$$

$$
\frac{1}{2}\left(\underline{E}_{b 1}+\underline{E}_{b 2}\right)=-\frac{2\left[Z_{1 M(A)}+s_{1,2(A)}\right]}{\sqrt{\underline{\sigma}_{1}^{2}+\underline{a}^{2}}}-\frac{2\left[Z_{1 M(B)}+s_{1,2(B)}\right]}{\sqrt{\underline{\sigma}_{2}^{2}+\underline{a}^{2}}}+\frac{1}{2 \underline{a}}+\frac{Z_{n A} Z_{n B}}{\underline{\sigma}_{1}+\underline{\sigma}_{2}}+2 \underline{T}_{m}
$$

where $t_{c A}$ and $t_{c B}$ are calculated, respectively, with the equations (S52) and (S54). This system has 5 equations with 5 unknowns, which are $E_{b 1}, E_{b 2}, \sigma_{1}, \sigma_{2}$ and $a$. The value of $T_{m}$ is very small; it is chosen from the condition of minimum value of the total energy. All the parameters and expressions which are used in calculations 


\section{Molecular Physics}

Table S5. Coordinates of average positions of particles, effective screening coefficients, effective order numbers and solutions of the equations describing the behavior of $\mathrm{BeH}$ molecule.

\begin{tabular}{|c|c|}
\hline $\begin{array}{l}\text { Particle coordinates; quantum numbers } \\
\text { Calculated and experimental values }\end{array}$ & Screening coefficients; order numbers \\
\hline$\overline{n_{A}(0,0,0,) ; n_{B}\left(\underline{\sigma}_{1}+\underline{\sigma}_{2}, 0,0,\right)}$ & $s_{1, A 1}=s_{A 3, A 1}=0.83882[27]$ \\
\hline $\begin{array}{l}\text { Phase A: } \\
e_{1}\left[\frac{3}{4} \underline{\sigma}_{1}, \frac{3}{4} \underline{a}, 0\right] ; e_{2}\left[\frac{3}{4} \sigma_{1},-\frac{3}{4} \underline{a}, 0\right]\end{array}$ & $\begin{array}{c}s_{A 1,1}=s_{A 1, A 3}=0.00221203[27] \\
s_{A 1, A 2}=0.25\end{array}$ \\
\hline Phase B: & $s_{1,2(A)}=\sqrt{\underline{\sigma}_{1}^{2}+\underline{a}^{2}} /(4 \underline{a})$ \\
\hline$e_{1}\left[\underline{\sigma}_{1}+\frac{1}{4} \underline{\sigma}_{1}, \frac{3}{4} \underline{a}, 0\right] ; e_{2}\left[\underline{\sigma}_{1}+\frac{1}{4} \underline{\sigma}_{1},-\frac{3}{4} \underline{a}, 0\right]$ & $s_{1, A 3}=(\sqrt{2} / 4) / \sqrt{1+\frac{\underline{\sigma}_{1}}{\sqrt{{\underline{\sigma_{1}^{2}+\underline{a}^{2}}}}}}$ \\
\hline$e_{3}\left(-\underline{\widetilde{r}}_{A 3}, 0,0\right)$ & $s_{1,2(B)}=\sqrt{{\underline{\sigma_{2}^{2}+\underline{a}^{2}}}^{2}} /(4 \underline{a})$ \\
\hline$n_{1(A)}=n_{2(A)}=2 ; n_{1(B)}=n_{2(B)}=1$ & $Z_{1(A)}=Z_{A}-2 s_{1, A 1}-s_{1,2(A)}-s_{1, A 3}$ \\
\hline $\begin{array}{l}n_{A 1}=n_{A 2}=1 ; n_{A 3}=2 \\
\underline{E}_{b 1}=-2.51578 ; \underline{E}_{b 2}=2.11007\end{array}$ & $\begin{array}{c}Z_{1 M(A)}=Z_{A}-2-s_{1,2(A)}-s_{1, A 3} \\
Z_{A 1}=Z_{A}-s_{A 1, A 2}-3 s_{A 1,1}\end{array}$ \\
\hline $\begin{array}{l}\underline{\sigma}_{1}=1.34462 ; \underline{\sigma}_{2}=0.0149424 \\
\underline{a}=0.979054 ; \underline{T}_{m}=.015\end{array}$ & $\begin{array}{c}Z_{A 3}=Z_{A}-2 s_{1, A 1}-2 s_{1, A 3} \\
Z_{1(B)}=Z_{B}-s_{1,2(B)}\end{array}$ \\
\hline$\underline{\bar{S}}_{E i A}=29.337 \overline{7}[35] ; \underline{S}_{E i B}=1$ & $Z_{1 M(B)}=Z_{B}-s_{1,2(B)}$ \\
\hline$\underline{D}_{0}^{0}=0.1495[36]$ & $\underline{\widetilde{r}}_{A 3}=\frac{3}{Z_{A}-2 s_{A 3, A 1}}$ \\
\hline$r_{0}=1.4389 A ; r_{e}=1.3426 A[29]$ & $Z_{n A}=Z_{A}-2-\frac{\left(\underline{\sigma}_{1}+\underline{\sigma}_{2}\right)^{2}}{\left(\underline{\sigma}_{1}+\underline{\sigma}_{2}+\underline{\widetilde{r}}_{A 3}\right)^{2}}$ \\
\hline $\begin{array}{l}\mu_{e}=0.192613 \quad D \\
\mu_{e H F}=(0.232,0.595) \quad D\end{array}$ & $Z_{n B}=Z_{B}^{1}$ \\
\hline
\end{tabular}

are shown in Table S5. The screening coefficients between the $1 s$ electrons and the $e_{1}, e_{2}$ and $e_{A 3}$ electrons are identical to $s_{31 e}$ and $s_{13 e}$, which have been calculated in Ref. [27] for the $1 s^{2} 2 s^{2}$ state of beryllium atom. In the case of heteronuclear molecules, the values of $Z_{n A}$ and $Z_{n B}$ are calculated with equation (39) from paper in which, instead of $(2 \sigma)^{2}$, we consider the expression $\left(\sigma_{1}+\sigma_{2}\right)^{2}$.

The normalized value of the total energy is calculated with the aid of equation (41) from paper, which can be written for $\mathrm{BeH}$ molecule, when the electrons are situated at points $A_{1}$ and $A_{2}$, taking into account the symmetry of the system (namely $\underline{U}_{e_{1} n_{A}}=\underline{U}_{e_{2} n_{A}}$ and $\underline{U}_{e_{1} n_{B}}=\underline{U}_{e_{2} n_{B}}$ ), as follows:

$$
\begin{gathered}
\underline{E}=\underline{E}_{A 3}+2 \underline{E}_{A 1}+2 \underline{U}_{e_{1} n_{A}}+2 \underline{U}_{e_{1} n_{B}}+\underline{U}_{e_{1} e_{2}}+\underline{U}_{e_{A 3} n_{B}}+\underline{U}_{n_{A} n_{B}}+\underline{E}_{m 1 s}=(\mathrm{S} 65) \\
-\frac{Z_{A 3}^{2}}{n_{A 3}^{2}}-2 \frac{Z_{A 1}^{2}}{n_{A 1}^{2}}-\frac{2\left[Z_{1 M(A)}+s_{1,2(A)}\right]}{\sqrt{\underline{\sigma}_{1}^{2}+\underline{a}^{2}}}-\frac{2\left[Z_{1 M(B)}+s_{1,2(B)}\right]}{\sqrt{\underline{\sigma}_{2}^{2}+\underline{a}^{2}}}+\frac{1}{2 \underline{a}}- \\
\frac{Z_{B}}{\underline{\sigma}_{1}+\underline{\sigma}_{2}+\underline{\widetilde{r}}_{A 3}}+\frac{\left(Z_{A}-2\right) Z_{B}}{\underline{\sigma}_{1}+\underline{\sigma}_{2}}+\frac{Z_{A 1}^{3 / 2}}{8 n_{A 1}^{3}}+2 \underline{T}_{m}
\end{gathered}
$$

The values of $\underline{E}, \underline{E}_{H F}$ and $\underline{E}_{\exp }$ are given in Table 1 of paper. The relative errors corresponding to $\underline{E}$ and $\underline{E}_{H F}$ are, respectively, equal to 0.002 and 0.006 . The electric dipole moment is given by the following relation:

$$
\mu_{e}=-5.08315\left[-2 \underline{\sigma}_{1}+Z_{B}\left(\underline{\sigma}_{1}+\underline{\sigma}_{2}\right)+\underline{\widetilde{r}}_{A 3}\right]
$$

The values of $r_{0}, r_{e}, \mu_{e}$ and the domain of the values of $\mu_{e H F}$ [29] are shown in Table S5.

\section{S2.5. BH molecule}

The structure of this molecule is shown in Fig. S3(b). This molecule contains two electrons, denoted by $e_{A 3}$ and $e_{A 4}$ which do not participate to the bond. The geometrical structure of the molecule results from the system (S33), (S36) and 


\section{Molecular Physics}

Molecular Physics

Table S6. Coordinates of average positions of particles, effective screening coefficients, effective order numbers and solutions of the equations describing the behavior of $B H$ molecule.

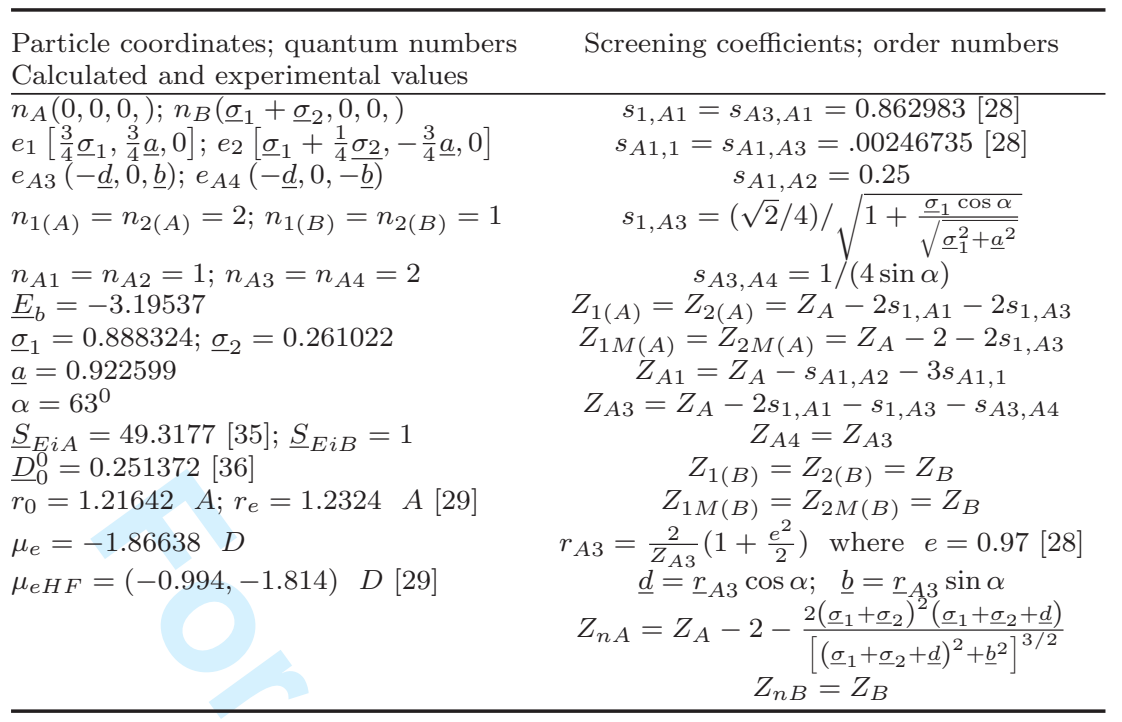

(S37), which can be written in normalized form, as follows:

$$
\begin{gathered}
\underline{E}_{b}=-\frac{Z_{1(A)}^{2}}{n_{1(A)}^{2}}-\frac{Z_{2(B)}^{2}}{n_{2(B)}^{2}}-\frac{4 Z_{1 M(B)}}{\sqrt{9 \underline{a}^{2}+\left(\underline{\sigma}_{1}+4 \underline{\sigma}_{2}\right)^{2}}}-\frac{4 Z_{1 M(A)}}{\sqrt{9 \underline{a}^{2}+\left(\underline{\sigma}_{2}+4 \underline{\sigma}_{1}\right)^{2}}}+ \\
\frac{4}{\sqrt{36 \underline{a}^{2}+\left(\underline{\sigma}_{1}+\underline{\sigma}_{2}\right)^{2}}+\frac{Z_{n A} Z_{n B}}{\underline{\sigma}_{1}+\underline{\sigma}_{2}}} \\
\underline{E}_{b}=-\frac{\left[n_{1(A)}+n_{1(B)}\right] \sqrt{Z_{1 M(A)}}}{\left(\underline{a}^{2}+\underline{\sigma}_{1}^{2}\right)^{3 / 4}} \\
\underline{E}_{b}=-\frac{\left[n_{1(A)}+n_{1(B)}\right] \sqrt{Z_{1 M(B)}}}{\left(\underline{a}^{2}+\underline{\sigma}_{2}^{2}\right)^{3 / 4}} \\
\underline{E}_{b}=-\frac{2 Z_{1 M(A)}}{\sqrt{\underline{\sigma}_{1}^{2}+\underline{a}^{2}}}-\frac{2 Z_{1 M(B)}}{\sqrt{\underline{\sigma}_{2}^{2}+\underline{a}^{2}}}+\frac{1}{2 \underline{a}}+\frac{Z_{n A} Z_{n B}}{\underline{\sigma}_{1}+\underline{\sigma}_{2}}
\end{gathered}
$$

The system (S67)-(S70) has 4 equations with 4 unknowns, which are $\underline{E_{b}}, \underline{\sigma_{1}}, \underline{\sigma_{2}}$ and $\underline{a}$. The expressions of the screening coefficients, order numbers and quantum numbers are given in Table S6. The screening coefficients between the $1 s$ electrons and the $e_{1}, e_{2}, e_{A 3}$ and $e_{A 4}$ electrons, namely $s_{1, A 1}, s_{A 1,1}, s_{A 3, A 1}, s_{A 1, A 3}$, and so on, are identical, respectively to $s_{31 e}$ and $s_{13 e}$, which have been calculated in Ref. [28] for the boron atom. The angle $\alpha$ is a parameter. We perform the calculation for a lot of values of this angle and choose the value for which the total energy is minimum. This value is given in Table S6.

Taking into account the symmetry of the system, the total energy results from 
the following relation [see Fig. S3(b)]:

$$
\begin{aligned}
& \underline{E}=\underline{E}_{1}+\underline{E}_{2}+2 \underline{E}_{A 3}+2 \underline{E}_{A 1}+\underline{U}_{e_{1} e_{2}}+\underline{U}_{e_{1} n_{B}}+\underline{U}_{e_{2} n_{A}}+2 \underline{U}_{e_{A 3} e_{2}}+ \\
& 2 \underline{U}_{e_{A 3} n_{B}}+\underline{U}_{n_{A} n_{B}}+2 \underline{E}_{m 1 s}=-\frac{Z_{1(A)}^{2}}{n_{1(A)}^{2}}-\frac{Z_{2(B)}^{2}}{n_{2(B)}^{2}}-2 \frac{Z_{A 3}^{2}}{n_{A 3}^{2}}-2 \frac{Z_{A 1}^{2}}{n_{A 1}^{2}}+ \\
& \frac{1}{\sqrt{\left(\frac{1}{4} \underline{\sigma}_{1}+\frac{1}{4} \underline{\sigma}_{2}\right)^{2}+\left(\frac{3}{2} \underline{a}\right)^{2}}}-\frac{Z_{B}}{\sqrt{\left(\frac{1}{4} \underline{\sigma}_{1}+\underline{\sigma}_{2}\right)^{2}+\left(\frac{3}{4} \underline{a}\right)^{2}}}- \\
& \frac{Z_{A}-2}{\sqrt{\left(\frac{1}{4} \underline{\sigma}_{2}+\underline{\sigma}_{1}\right)^{2}+\left(\frac{3}{4} \underline{a}\right)^{2}}}+\frac{2}{\sqrt{\left(\underline{\sigma}_{1}+\frac{1}{4} \underline{\sigma}_{2}+\underline{d}\right)^{2}+\left(\frac{3}{4} \underline{a}\right)^{2}+\underline{b}^{2}}}- \\
& \frac{2 Z_{B}}{\sqrt{\left(\underline{\sigma}_{1}+\underline{\sigma}_{2}+\underline{d}\right)^{2}+\underline{b}^{2}}}+\frac{\left(Z_{A}-2\right) Z_{B}}{\underline{\sigma}_{1}+\underline{\sigma}_{2}}+\frac{Z_{A 1}^{3 / 2}}{8 n_{A 1}^{3}}
\end{aligned}
$$

$$
\mu_{e}=-5.08315\left[-2 \underline{\sigma}_{1}+Z_{B}\left(\underline{\sigma}_{1}+\sigma_{2}\right)+2 \underline{d}\right]
$$

The values of $\underline{E}, \underline{E}_{H F}$ and $\underline{E}_{\text {exp }}$ are given in Table 1 of paper. The relative errors corresponding to $\underline{E}$ and $\underline{E}_{H F}$ are, respectively, equal to 0.002 and 0.006 . The electric dipole moment is given by the following relation:

The values of $r_{0}, r_{e}, \mu_{e}$ and the domain of the values of $\mu_{e H F}$ [29] are shown in Table S6.

\section{S2.6. $\mathrm{CH}$ molecule}

The structure of this molecule is shown in Fig. S3(c). This molecule contains three electrons, denoted by $e_{A 3}, e_{A 4}$ and $e_{A 5}$ which do not participate to the bond. The geometrical structure of the molecule results from the system (S67)-(S70). All the data necessary in calculations, together with the results of the calculations, are given in Table S7.

The normalized value of the total energy is calculated with the aid of equation 


\section{Molecular Physics}

Molecular Physics

Table S7. Coordinates of average positions of particles, effective screening coefficients, effective order numbers and solutions of the equations describing the behavior of $\mathrm{CH}$ molecule.

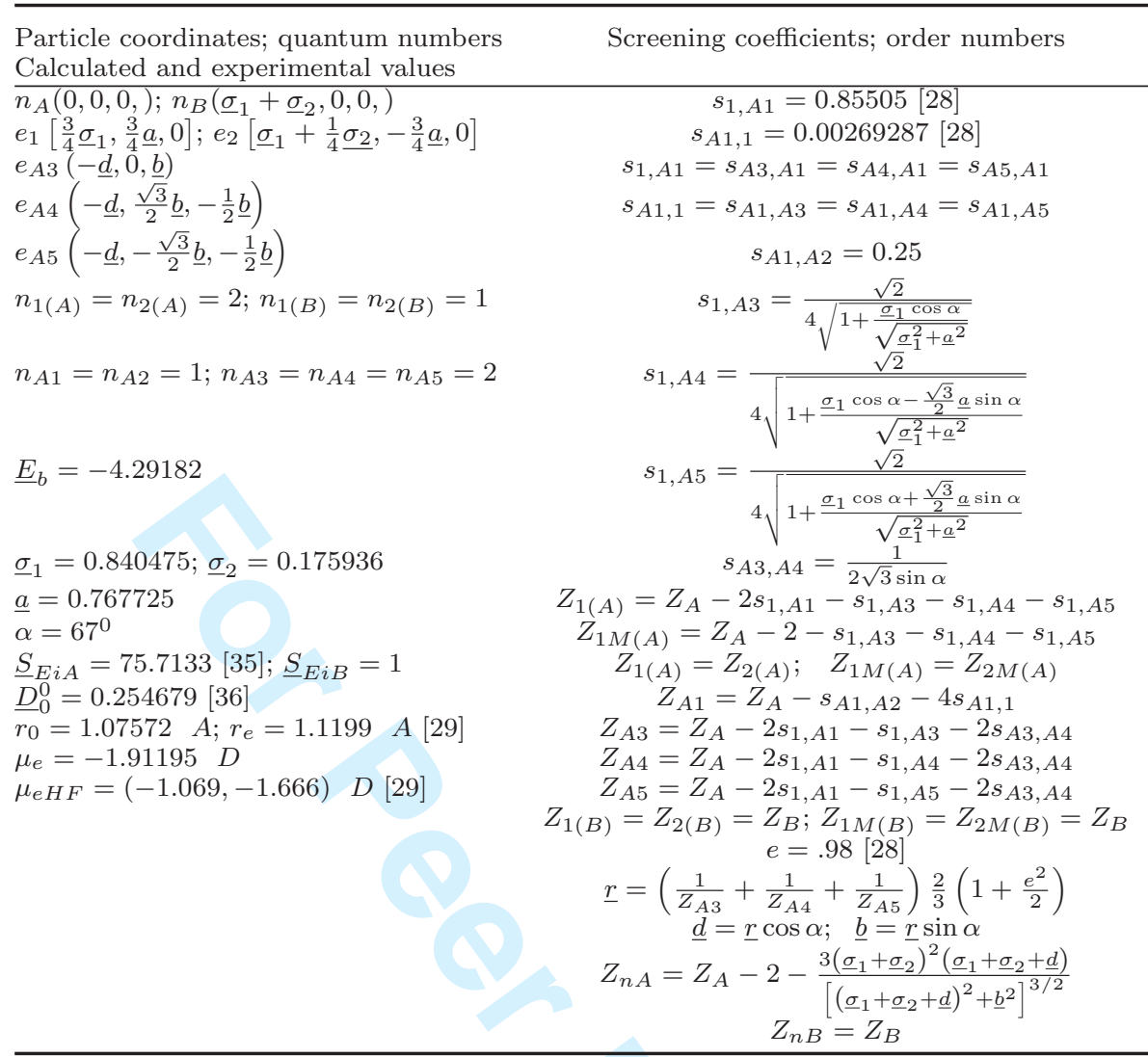

(41), which can be written as follows:

$$
\begin{aligned}
& \underline{E}=\underline{E}_{1}+\underline{E}_{2}+\underline{E}_{A 3}+\underline{E}_{A 4}+\underline{E}_{A 5}+2 \underline{E}_{A 1}+\underline{U}_{e_{1} e_{2}}+\underline{U}_{e_{1} n_{B}}+\underline{U}_{e_{2} n_{A}}+ \\
& \underline{U}_{e_{A 3} e_{2}}+\underline{U}_{e_{A 4} e_{2}}+\underline{U}_{e_{A 5} e_{2}}+3 \underline{U}_{e_{A 3} n_{B}}+\underline{U}_{n_{A} n_{B}}+2 \underline{E}_{m 1 s}=-\frac{Z_{1(A)}^{2}}{n_{1(A)}^{2}}- \\
& \frac{Z_{2(B)}^{2}}{n_{2(B)}^{2}}-\frac{Z_{A 3}^{2}}{n_{A 3}^{2}}-\frac{Z_{A 4}^{2}}{n_{A 3}^{2}}-\frac{Z_{A 5}^{2}}{n_{A 3}^{2}}-2 \frac{Z_{A 1}^{2}}{n_{A 1}^{2}}+\frac{1}{\sqrt{\left(\frac{1}{4} \underline{\sigma}_{1}+\frac{1}{4} \underline{\sigma}_{2}\right)^{2}+\left(\frac{3}{2} \underline{a}\right)^{2}}}- \\
& \frac{Z_{B}}{\sqrt{\left(\frac{1}{4} \underline{\sigma}_{1}+\underline{\sigma}_{2}\right)^{2}+\left(\frac{3}{4} \underline{a}\right)^{2}}}-\frac{Z_{A}-2}{\sqrt{\left(\frac{1}{4} \underline{\sigma}_{2}+\underline{\sigma}_{1}\right)^{2}+\left(\frac{3}{4} \underline{a}\right)^{2}}}+ \\
& \frac{1}{\sqrt{\left(\underline{\sigma}_{1}+\frac{1}{4} \underline{\sigma}_{2}+\underline{d}\right)^{2}+\left(\frac{3}{4} \underline{a}\right)^{2}+\underline{b}^{2}}}+ \\
& \frac{1}{\sqrt{\left(\underline{\sigma}_{1}+\frac{1}{4} \underline{\sigma}_{2}+\underline{d}\right)^{2}+\left(\frac{3}{4} \underline{a}+\frac{\sqrt{3}}{2} \underline{b}\right)^{2}+\left(\frac{1}{2} \underline{b}\right)^{2}}}+ \\
& \frac{1}{\sqrt{\left(\underline{\sigma}_{1}+\frac{1}{4} \underline{\sigma}_{2}+\underline{d}\right)^{2}+\left(\frac{3}{4} \underline{a}-\frac{\sqrt{3}}{2} \underline{b}\right)^{2}+\left(\frac{1}{2} \underline{b}\right)^{2}}}- \\
& \frac{3 Z_{B}}{\sqrt{\left(\underline{\sigma}_{1}+\underline{\sigma}_{2}+\underline{d}\right)^{2}+\underline{b}^{2}}}+\frac{\left(Z_{A}-2\right) Z_{B}}{\underline{\sigma}_{1}+\underline{\sigma}_{2}}+\frac{Z_{A 1}^{3 / 2}}{8 n_{A 1}^{3}}
\end{aligned}
$$


The values of $\underline{E}, \underline{E}_{H F}$ and $\underline{E}_{\text {exp }}$ are given in Table 1 of paper. The relative errors corresponding to $\underline{E}$ and $\underline{E}_{H F}$ are, respectively, equal to 0.004 and 0.005 . The electric dipole moment is given by the following relation:

$$
\mu_{e}=-5.08315\left[-2 \underline{\sigma}_{1}+Z_{B}\left(\underline{\sigma}_{1}+\underline{\sigma}_{2}\right)+3 \underline{d}\right]
$$

The values of $\alpha$, corresponding to the minimum value of $\underline{E}, r_{0}, r_{e}, \mu_{e}$ and the domain of the values of $\mu_{e H F}$ are shown in Table S7.

The analysis of the Table 1 from paper shows that the relative error of our calculations, in the most unfavorable cases, is, as in the case of atoms [27, 28], of the order 0.005. In Section 6 of Ref. [27] we presented a brief error analysis of our calculation method, arriving to the conclusion that the main source of error comes from the approximations made when calculating the correction term $E_{m 1 s}$. This analysis is valid also for the present paper.

S3. MATHEMATICA 7 scripts for the calculation of the $L i_{2}, B e_{2}, B_{2}, C_{2}$, $\mathrm{LiH}, \mathrm{BeH}, \mathrm{BH}$ and $\mathrm{CH}$ molecules.

This material contains all the numerical calculations, necessary in the paper.

\section{S3.1. Mathematica 7 script for the calculation of $\mathrm{Li}_{2}$.}

The calculation algorithm is described in Section 4. We have the following equivalences between the notations from paper and the symbols which are used in program: $Z_{A} \equiv Z A, s_{1, A 1} \equiv s 1 A 1, s_{A 1,1} \equiv s A 11, s_{A 1, A 2} \equiv s A 1 A 2, Z_{1} \equiv Z 1$, $Z_{1 M} \equiv Z 1 M, Z_{n A} \equiv Z n A, \underline{E} \equiv$ Etotal, $\underline{E}_{b} \equiv E B, r_{0} \equiv r 0, \underline{D}_{0}^{0} \equiv D 00$, $\underline{S}_{E i A} \equiv S E i A, \underline{E}_{e x p} \equiv \operatorname{Eexp}, r_{e} \equiv r e, \underline{a} \equiv a, \underline{\sigma} \equiv s$.

The output data, given at the end of the script are, respectively, $\underline{E}, \underline{E}_{\text {exp }}, r_{0}$ and $r_{e}$.

Input data.

$\mathrm{ZA}=3$

$\mathrm{s} 1 \mathrm{~A} 1=0.854942$

$\mathrm{sA11}=0.0013792$

$\mathrm{sA} 1 \mathrm{~A} 2=.25$

$\mathrm{Z} 1=\mathrm{ZA}-2 * \mathrm{~s} 1 \mathrm{~A} 1$

$\mathrm{Z} 1 \mathrm{M}=\mathrm{ZA}-2$

$\mathrm{ZnA}=\mathrm{ZA}-2$

Etotal $=\mathrm{EB}-4 *(\mathrm{ZA}-\mathrm{sA} 1 \mathrm{~A} 2-\mathrm{sA} 11)^{2}+\frac{(\mathrm{ZA}-\mathrm{sA1A2}-\mathrm{sA} 11)^{\frac{3}{2}}}{4}$

$\mathrm{r} 0=2 * s * 2 * .529177 A$;

Experimental data.

$\mathrm{D} 00=\frac{1.046}{13.60535}$

$\mathrm{SEiA}=\frac{5.39172+75.64018+122.45429}{13.60535}$

$\operatorname{Eexp}=-2 * \mathrm{SEiA}-\mathrm{D} 00$;

re $=2.673 A$;

System of equations

$\mathrm{Eq} 1=\mathrm{EB}+\frac{\mathrm{Z1}^{2}}{2}+\frac{8 * \mathrm{Z} 1 \mathrm{M}}{\sqrt{9 * a^{2}+25 * s^{2}}}-\frac{2}{\sqrt{9 * a^{2}+s^{2}}}-\frac{\mathrm{ZnA}^{2}}{2 * s} ;$

$\mathrm{Eq} 2=\mathrm{EB}+\frac{4 \sqrt{\mathrm{Z} 1 \mathrm{M}}}{\left(s^{2}+a^{2}\right)^{\frac{3}{4}}}$

$\mathrm{Eq} 3=\mathrm{EB}+\frac{4 * \mathrm{Z} 1 \mathrm{M}}{\sqrt{s^{2}+a^{2}}}-\frac{\mathrm{ZnA}^{2}}{2 * s}-\frac{1}{2 a} ;$

The solution of the system of equations 
Sol $=$ FindRoot $[\{\mathrm{Eq} 1==0, \mathrm{Eq} 2==0, \mathrm{Eq} 3==0\},\{\{\mathrm{EB}, 1\},\{s, 1.2\},\{a, 2.5\}\}]$

$\{\mathrm{EB} \rightarrow-1.0115, s \rightarrow 1.46474, a \rightarrow 2.02683\}$

Verification.

$N[\mathrm{Eq} 1] /$. Sol

$N[\mathrm{Eq} 2] /$. Sol

$N[\mathrm{Eq} 3] /$. Sol

0 .

$2.220446049250313^{* \wedge}-16$

$4.440892098500626^{* \wedge}-16$

Calculation of other quantities.

Etotal/.Sol

Eexp/.Sol

r0/.Sol

re/.Sol

$-30.0919$

$-29.9896$

$3.10043 A$

$2.673 A$

S3.2. Mathematica 7 script for the calculation of $\mathrm{Be}_{2}$.

We have the following equivalences between the notations from paper and the symbols which are used in program: $Z_{A} \equiv Z A, s_{1, A 1} \equiv s 1 A 1, s_{A 1,1} \equiv s A 11$, $s_{1, A 3 \equiv s 1 A 3}, s_{A 1, A 2} \equiv s A 1 A 2, Z_{1} \equiv Z 1, Z_{1 M} \equiv Z 1 M, Z_{A 3} \equiv Z A 3, Z_{A 1} \equiv Z A 1$, $\underline{r}_{A 3} \equiv r A 3, Z_{n A} \equiv Z n A, \underline{E} \equiv$ Etotal, $\underline{E}_{b} \equiv E B, r_{0} \equiv r 0, \underline{D}_{0}^{0} \equiv D 00, \underline{S}_{E i A} \equiv$ $S E i A, \underline{E}_{\exp } \equiv \operatorname{Eexp}, r_{e} \equiv r e, \underline{a} \equiv a, \underline{\sigma} \equiv s$.

The output data, given at the end of the script are, respectively, $\underline{E}, \underline{E}_{e x p}, r_{0}$ and $r_{e}$.

Input data.

$\mathrm{ZA}=4$

$\mathrm{s} 1 \mathrm{~A} 1=0.83882$

$\mathrm{sA} 11=.00221203$

$\mathrm{s} 1 \mathrm{~A} 3=\frac{\sqrt{2}}{4 * \sqrt{1+\frac{s}{\sqrt{s^{2}+a^{2}}}}}$

$\mathrm{sA} 1 \mathrm{~A} 2=.25$

$\mathrm{Z} 1=\mathrm{ZA}-2 \mathrm{~s} 1 \mathrm{~A} 1-\mathrm{s} 1 \mathrm{~A} 3$

$\mathrm{Z} 1 \mathrm{M}=\mathrm{ZA}-2-\mathrm{s} 1 \mathrm{~A} 3$

$\mathrm{ZA} 3=\mathrm{Z} 1$

$\mathrm{ZA} 1=\mathrm{ZA}-\mathrm{sA} 1 \mathrm{~A} 2-2 * \mathrm{sA} 11$

$\mathrm{rA} 3=\frac{3}{\mathrm{ZA} 3}$

$\mathrm{ZnA}=\mathrm{ZA}-2-\frac{(2 s)^{2}}{(2 s+\mathrm{rA} 3)^{2}}$

Etotal $=-\frac{\mathrm{Z1}^{2}}{2}-\frac{\mathrm{ZA}^{2}}{2}-4 * \mathrm{ZA1}^{2}+\frac{1}{2 \sqrt{\left(\frac{1}{4} * s\right)^{2}+\left(\frac{3}{4} * a\right)^{2}}}-\frac{2 *(\mathrm{ZA}-2)}{\sqrt{\left(\frac{5}{4} * s\right)^{2}+\left(\frac{3}{4} * a\right)^{2}}}+\frac{2}{\sqrt{\left(\frac{5}{4} * s+\mathrm{rA} 3\right)^{2}+\left(\frac{3}{4} * a\right)^{2}}}-$

$\frac{2 *(\mathrm{ZA}-2)}{2 * s+\mathrm{rA} 3}+\frac{1}{2 * s+2 * \mathrm{rA} 3}+\frac{(\mathrm{ZA}-2)^{2}}{2 * s}+\frac{\mathrm{ZA} 1 \frac{3}{2}}{4}$

$\mathrm{r} 0=2 * s * 2 * .529177 A$

Experimental data.

$\mathrm{D} 00=.1643$

$\mathrm{SEiA}=\frac{9.3227+18.21116+153.89661+217.71865}{13.60535}$;

Eexp $=-2 * \mathrm{SEiA}-\mathrm{D} 00$;

re $=2.460 A$;

System of equations. 
$\mathrm{Eq} 1=\mathrm{EB}+\frac{\mathrm{Z1}^{2}}{2}+\frac{8 * \mathrm{Z1M}}{\sqrt{9 * a^{2}+25 * s^{2}}}-\frac{2}{\sqrt{9 * a^{2}+s^{2}}}-\frac{\mathrm{ZnA}^{2}}{2 * s}$

$\mathrm{Eq} 2=\mathrm{EB}+\frac{4 \sqrt{\mathrm{Z1M}}}{\left(s^{2}+a^{2}\right)^{\frac{3}{4}}}$

$\mathrm{Eq} 3=\mathrm{EB}+\frac{4 * \mathrm{Z} 1 \mathrm{M}}{\sqrt{s^{2}+a^{2}}}-\frac{\mathrm{ZnA}^{2}}{2 * s}-\frac{1}{2 a}$

The solution of the system of equations.

$\mathrm{Sol}=$ FindRoot $[\{\mathrm{Eq} 1==0, \mathrm{Eq} 2==0, \mathrm{Eq} 3==0\},\{\{\mathrm{EB},-2\},\{s, 1\},\{a, 1\}\}]$

$\{\mathrm{EB} \rightarrow-2.39104, s \rightarrow 1.04502, a \rightarrow 1.32709\}$

Verification.

$N[\mathrm{Eq} 1] /$. Sol

$N[\mathrm{Eq} 2] /$. Sol

$N[\mathrm{Eq} 3] /$. Sol

$2.220446049250313^{* \wedge}-16$

$-4.440892098500626^{* \wedge}-16$

$-4.440892098500626^{* \wedge}-16$

Calculation of other quantities.

Etotal/.Sol

Eexp/.Sol

r0/.Sol

re/.Sol

$-58.7676$

$-58.8396$

$2.212 \mathrm{~A}$

$2.46 \mathrm{~A}$

S3.3. Mathematica 7 script for the calculation of $B_{2}$.

The equivalences between the notations from paper and the symbols which are used in program are $Z_{A} \equiv Z A, s_{1, A 1} \equiv s 1 A 1, s_{A 1,1} \equiv s A 11, s_{A 1, A 2} \equiv s A 1 A 2$, $\alpha \equiv$ alpha, $s_{1, A 3 \equiv s 1 A 3}, s_{A 3, A 4} \equiv s A 3 A 4, Z_{1} \equiv Z 1, Z_{1 M} \equiv Z 1 M, Z_{A 3} \equiv Z A 3$, $Z_{A 1} \equiv Z A 1, \underline{r} \equiv r, \underline{d} \equiv d, \underline{b} \equiv b, Z_{n A} \equiv Z n A, \underline{E} \equiv$ Etotal, $\underline{E}_{b} \equiv E B, r_{0} \equiv r 0$, $\underline{D}_{0}^{0} \equiv D 00, \underline{S}_{E i A} \equiv S E i A, \underline{E}_{\exp } \equiv \operatorname{Eexp}, r_{e} \equiv r e, \underline{a} \equiv a, \underline{\sigma} \equiv s$.

The output data, given at the end of the script are, respectively, $\underline{E}, \underline{E}_{e x p}, r_{0}$ and $r_{e}$.

Input data.

$\mathrm{ZA}=5$

$\mathrm{s} 1 \mathrm{~A} 1=0.862983$

$\mathrm{sA} 11=.00246735$;

$\mathrm{sA} 1 \mathrm{~A} 2=.25$

alpha $=63$;

al $=$ alpha $* \frac{\pi}{180}$

$\mathrm{s} 1 \mathrm{~A} 3=\frac{1}{4 * \sqrt{\frac{1}{2}+\frac{s * \operatorname{Cos}[a]]}{2 * \sqrt{s^{2}+a^{2}}}}}$

$\mathrm{sA} 3 \mathrm{~A} 4=\frac{1}{4 * \operatorname{Sin}[\mathrm{al}]}$;

$\mathrm{Z} 1=\mathrm{ZA}-2 * \mathrm{~s} 1 \mathrm{~A} 1-2 * \mathrm{~s} 1 \mathrm{~A} 3$;

$\mathrm{Z} 1 \mathrm{M}=\mathrm{ZA}-2-2 * \mathrm{~s} 1 \mathrm{~A} 3$

$\mathrm{ZA} 1=\mathrm{ZA}-\mathrm{sA} 1 \mathrm{~A} 2-3 * \mathrm{sA} 11$

$\mathrm{ZA} 3=\mathrm{ZA}-2 * \mathrm{~s} 1 \mathrm{~A} 1-\mathrm{s} 1 \mathrm{~A} 3-\mathrm{sA} 3 \mathrm{~A} 4$;

$r=\frac{3}{\mathrm{ZA} 3}$

$d=r * \operatorname{Cos}[\mathrm{al}]$

$b=r * \operatorname{Sin}[\mathrm{al}]$; 
$\mathrm{ZnA}=\mathrm{ZA}-2-\frac{2 *(2 s)^{2} *(2 * s+d)}{\left((2 * s+d)^{2}+b^{2}\right)^{\frac{3}{2}}}$

Etotal $=-\frac{\mathrm{Z1}^{2}}{2}-\mathrm{ZA3}^{2}-4 * \mathrm{ZA}^{2}+\frac{1}{2 * \sqrt{\left(\frac{1}{4} * s\right)^{2}+\left(\frac{3}{4} * a\right)^{2}}}-\frac{2 *(\mathrm{ZA}-2)}{\sqrt{\left(\frac{5}{4} * s\right)^{2}+\left(\frac{3}{4} * a\right)^{2}}}+$

$\frac{4}{\sqrt{\left(\frac{5}{4} * s+d\right)^{2}+\left(\frac{3}{4} * a\right)^{2}+b^{2}}}-\frac{4 *(\mathrm{ZA}-2)}{\sqrt{(d+2 * s)^{2}+b^{2}}}+\frac{1}{\sqrt{(s+d)^{2}+b^{2}}}+\frac{1}{s+d}+\frac{(\mathrm{ZA}-2)^{2}}{2 * s}+\frac{\mathrm{ZA} 1^{\frac{3}{2}}}{4}$

$\mathrm{r} 0=2 * s * 2 * .529177 A$;

Experimental data.

$\mathrm{D} 00=\frac{3.02}{13.60535}$

$\mathrm{SEiA}=\frac{8.29803+25.15484+37.93064+259.37521+340.22580}{13.60535}$

$\mathrm{Eexp}=-2 * \mathrm{SEiA}-\mathrm{D} 00$;

re $=1.5900 A$;

System of equations.

$\mathrm{Eq} 1=\mathrm{EB}+\frac{\mathrm{Z1}^{2}}{2}+\frac{8 * \mathrm{Z} 1 \mathrm{M}}{\sqrt{9 * a^{2}+25 * s^{2}}}-\frac{2}{\sqrt{9 * a^{2}+s^{2}}}-\frac{\mathrm{ZnA}^{2}}{2 * s} ;$

$\mathrm{Eq} 2=\mathrm{EB}+\frac{4 \sqrt{\mathrm{Z1M}}}{\left(s^{2}+a^{2}\right)^{\frac{3}{4}}}$

$\mathrm{Eq} 3=\mathrm{EB}+\frac{4 * \mathrm{Z} 1 \mathrm{M}}{\sqrt{s^{2}+a^{2}}}-\frac{\mathrm{ZnA}^{2}}{2 * s}-\frac{1}{2 a}$

The solution of the system of equations.

$\mathrm{Sol}=$ FindRoot $[\{\mathrm{Eq} 1==0, \mathrm{Eq} 2==0, \mathrm{Eq} 3==0\},\{\{\mathrm{EB}, 1\},\{s, 1\},\{a, 1\}\}]$

$\{\mathrm{EB} \rightarrow-3.857, s \rightarrow 0.844851, a \rightarrow 1.07468\}$

Verification.

$N[\mathrm{Eq} 1] /$. Sol

$N[\mathrm{Eq} 2] /$. Sol

$N$ [Eq3]/.Sol

$1.3322676295501878^{* \wedge}-15$

$4.440892098500626^{* \wedge}-16$

0.

Calculation of other quantities.

Etotal/.Sol

Eexp/.Sol

r0/.Sol

re/.Sol

$-98.3341$

$-98.8574$

$1.7883 A$

$1.59 \mathrm{~A}$

S3.4. Mathematica 7 script for the calculation of $C_{2}$ with double bond.

The equivalences between the notations from paper and the symbols which are used in program are $Z_{A} \equiv Z A, s_{1, A 1} \equiv s 1 A 1, s_{A 1,1} \equiv s A 11, s_{A 1, A 2} \equiv s A 1 A 2, \alpha \equiv$ alpha, $s_{1,2} \equiv s 12, s_{1, A 3 \equiv s 1 A 3}, s_{1, A 4 \equiv s 1 A 4}, s_{A 3, A 4} \equiv s A 3 A 4, Z_{1} \equiv Z 1, Z_{1 M} \equiv Z 1 M$, $Z_{A 1} \equiv Z A 1, Z_{A 3} \equiv Z A 3, Z_{A 4} \equiv Z A 4, e \equiv$ excen, $\underline{r} \equiv r, \underline{d} \equiv d, \underline{b} \equiv b, Z_{n A} \equiv Z n A$, $\underline{E} \equiv$ Etotal, $\underline{E}_{b} \equiv E B, r_{0} \equiv r 0, \underline{D}_{0}^{0} \equiv D 00, \underline{S}_{E i A} \equiv S E i A, \underline{E}_{e x p} \equiv E \exp , r_{e} \equiv r e$, $\underline{a} \equiv a, \underline{\sigma} \equiv s$.

The output data, given at the end of the script are, respectively, $\underline{E}, \underline{E}_{e x p}, r_{0}$ and $r_{e}$.

Input data.

$\mathrm{ZA}=6$;

$\mathrm{s} 1 \mathrm{~A} 1=0.85505$

$\mathrm{sA11}=.00269287$ 


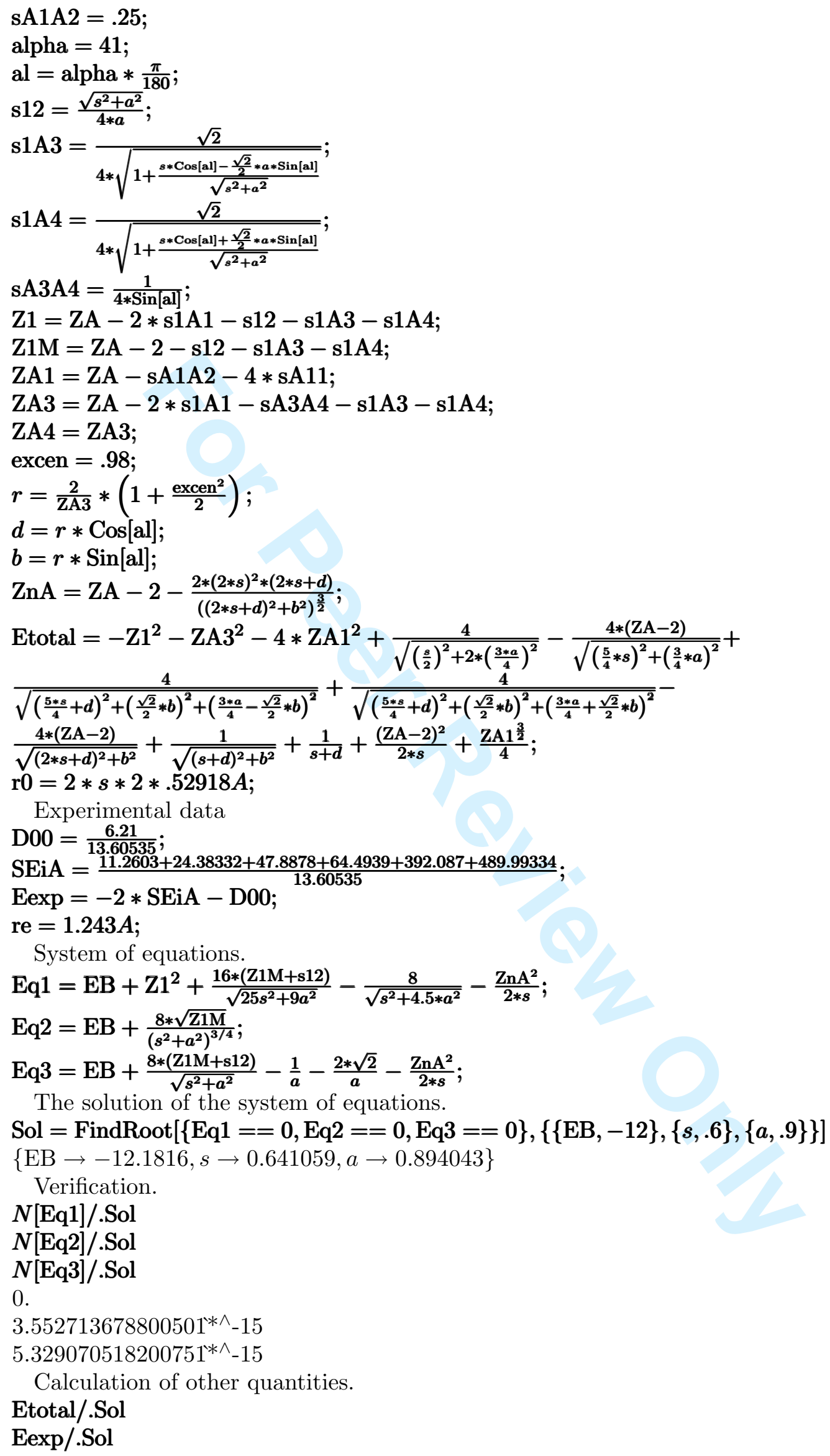


r0/.Sol

re/.Sol

$-151.148$

$-151.883$

$1.35694 A$

$1.243 A$

S3.5. Mathematica 7 script for the calculation of $C_{2}$ with triple bond.

The equivalences between the notations from paper and the symbols which are used in program are $Z_{A} \equiv Z A, s_{1, A 1} \equiv s 1 A 1, s_{A 1,1} \equiv s A 11, s_{A 1, A 2} \equiv s A 1 A 2$, $s_{1,2} \equiv s 12, s_{1, A 3 \equiv s 1 A 3}, Z_{1} \equiv Z 1, Z_{1 M} \equiv Z 1 M, Z_{A 1} \equiv Z A 1, Z_{A 3} \equiv Z A 3, e \equiv$ excen, $\underline{r}_{A 3} \equiv r A 3, Z_{n A} \equiv Z n A, \underline{E} \equiv$ Etotal, $\underline{E}_{b} \equiv E B, r_{0} \equiv r 0, \underline{D}_{0}^{0} \equiv D 00$, $\underline{S}_{E i A} \equiv S E i A, \underline{E}_{e x p} \equiv \operatorname{Eexp}, r_{e} \equiv r e, \underline{a} \equiv a, \underline{\sigma} \equiv s$.

The output data, given at the end of the script are, respectively, $\underline{E}, \underline{E}_{e x p}, r_{0}$ and $r_{e}$.

Input data.

$\mathrm{ZA}=6$;

$\mathrm{s} 1 \mathrm{~A} 1=0.85505$

$\mathrm{sA} 11=.00269287$

$\mathrm{sA} 1 \mathrm{~A} 2=.25$

$\mathrm{s} 12=\frac{\sqrt{2}}{4} / \sqrt{1-\frac{s^{2}-\frac{1}{2} * a^{2}}{s^{2}+a^{2}}}$

$\mathrm{s} 1 \mathrm{~A} 3=\frac{\sqrt{2}}{4} / \sqrt{1+\frac{s}{\sqrt{s^{2}+a^{2}}}}$

$\mathrm{Z} 1=\mathrm{ZA}-2 * \mathrm{~s} 1 \mathrm{~A} 1-2 * \mathrm{~s} 12-\mathrm{s} 1 \mathrm{~A} 3$

$\mathrm{Z} 1 \mathrm{M}=\mathrm{ZA}-2-2 * \mathrm{~s} 12-\mathrm{s} 1 \mathrm{~A} 3$

$\mathrm{ZA} 1=\mathrm{ZA}-\mathrm{sA} 1 \mathrm{~A} 2-4 * \mathrm{sA} 11$

$\mathrm{ZA} 3=\mathrm{ZA}-2 * \mathrm{~s} 1 \mathrm{~A} 1-3 * \mathrm{~s} 1 \mathrm{~A} 3$

excen $=.98$;

$\mathrm{rA3}=\frac{2}{\mathrm{ZA} 3} *\left(1+\frac{\text { excen }^{2}}{2}\right) ;$

$\mathrm{ZnA}=\mathrm{ZA}-2-\frac{(2 * s)^{2}}{(2 * s+\mathrm{rA} 3)^{2}}$

Etotal $=-6 * \frac{\mathrm{Z}^{2}}{4}-2 * \frac{\mathrm{ZA}^{2}}{4}-4 * \mathrm{ZA}^{2}+\frac{3}{\sqrt{\left(\frac{1}{2} * s\right)^{2}+\left(\frac{3}{2} * a\right)^{2}}}+$

$\frac{6}{\sqrt{\left(\frac{1}{2} * s\right)^{2}+\left(\frac{3}{4} * a\right)^{2}}}-\frac{6 *(\mathrm{ZA}-2)}{\sqrt{\left(\frac{5}{4} * s\right)^{2}+\left(\frac{3}{4} * a\right)^{2}}}+\frac{6}{\sqrt{\left(\frac{5 * s}{4}+\mathrm{rA} 3\right)^{2}+\left(\frac{3 * a}{4}\right)^{2}}}-$

$\frac{2 *(\mathrm{ZA}-2)}{2 * s+\mathrm{rA} 3}+\frac{1}{2 * s+2 * \mathrm{rA} 3}+\frac{(\mathrm{ZA}-2)^{2}}{2 * s}+\frac{\mathrm{ZA} 1 \frac{3}{2}}{4}$;

$\mathrm{r} 0=2 * s * 2 * .52918 A$

Experimental data

$\mathrm{D} 00=\frac{6.21}{13.60535}$

$\mathrm{SEiA}=\frac{11.2603+24.38332+47.8878+64.4939+392.087+489.99334}{13.60535} ;$

$\operatorname{Eexp}=-2 * \mathrm{SEiA}-\mathrm{D} 00$

re $=1.243 A$

System of equations.

$\mathrm{Eq} 1=\mathrm{EB}+6 * \frac{\mathrm{Z1}^{2}}{4}+\frac{24 *(\mathrm{Z} 1 \mathrm{M}+2 * \mathrm{~s} 12)}{\sqrt{9 * a^{2}+25 * s^{2}}}-\frac{6}{\sqrt{s^{2}+9 * a^{2}}}-\frac{24}{\sqrt{4 * s^{2}+9 * a^{2}}}-\frac{\mathrm{ZnA}^{2}}{2 * s}$

$\mathrm{Eq} 2=\mathrm{EB}+\frac{12 * \sqrt{\mathrm{Z1M}}}{\left(s^{2}+a^{2}\right)^{3 / 4}}$

$\mathrm{Eq} 3=\mathrm{EB}+\frac{12 *(\mathrm{Z} 1 \mathrm{M}+2 * \mathrm{~s} 12)}{\sqrt{s^{2}+a^{2}}}-\frac{6}{a * \sqrt{3}}-\frac{6}{a}-\frac{3}{2 * a}-\frac{\mathrm{ZnA}^{2}}{2 * s} ;$

The solution of the system of equations.

$\mathrm{Sol}=$ FindRoot $[\{\mathrm{Eq} 1==0, \mathrm{Eq} 2==0, \mathrm{Eq} 3==0\},\{\{\mathrm{EB}, 12\},\{s, .6\},\{a, .9\}\}]$ 
$\{\mathrm{EB} \rightarrow-17.1499, s \rightarrow 0.639379, a \rightarrow 0.942817\}$

Verification.

$N[\mathrm{Eq} 1] /$. Sol

$N[\mathrm{Eq} 2] /$. Sol

$N$ [Eq3]/.Sol

$-3.552713678800501^{* \wedge}-15$

0 .

$1.7763568394002505^{* \wedge}-14$

Calculation of other quantities.

Etotal/.Sol

Eexp/.Sol

r0/.Sol

re/.Sol

$-150.923$

$-151.883$

$1.35339 A$

$1.243 A$

S3.6. Mathematica 7 script for the calculation of LiH.

The equivalences between the notations from paper and the symbols which are used in program are $Z_{A} \equiv Z A, Z_{B} \equiv Z B, s_{1, A 1} \equiv s 1 A 1, s_{A 1,1} \equiv s A 11, s_{A 1, A 2} \equiv s A 1 A 2$, $s_{1,2(A)} \equiv s 12 A, s_{1,2(B)} \equiv s 12 B, Z_{1(A)} \equiv Z 1 A, Z_{1 M(A)} \equiv Z 1 M A, Z_{A 1} \equiv Z A 1$, $Z_{1(B)} \equiv Z 1 B, Z_{1 M(B)} \equiv Z 1 M B, Z_{n A} \equiv Z n A, Z_{n B} \equiv Z n B, a_{c A} \equiv a c A, a_{c B} \equiv$ $a c B, I_{A} \equiv I A, I_{B} \equiv I B, t_{c A} \equiv t c A, t_{c B} \equiv t c B, \underline{E} \equiv$ Etotal, $r_{0} \equiv r 0, \mu_{e} \equiv \mu e$, $D_{0}^{0} \equiv D 00, S_{E i A} \equiv S E i A, S_{E i B} \equiv S E i B, \underline{E}_{e x p} \equiv E \exp , r_{e} \equiv r e, \underline{E}_{b} \equiv E B, \underline{a} \equiv a$, $\underline{\sigma_{1}} \equiv s 1, \underline{\sigma_{2}} \equiv s 2$.

The output data, given at the end of the script are, respectively, $\underline{E}, \underline{E}_{\text {exp }}, \mu_{e}, r_{0}$ and $r_{e}$.

Input data.

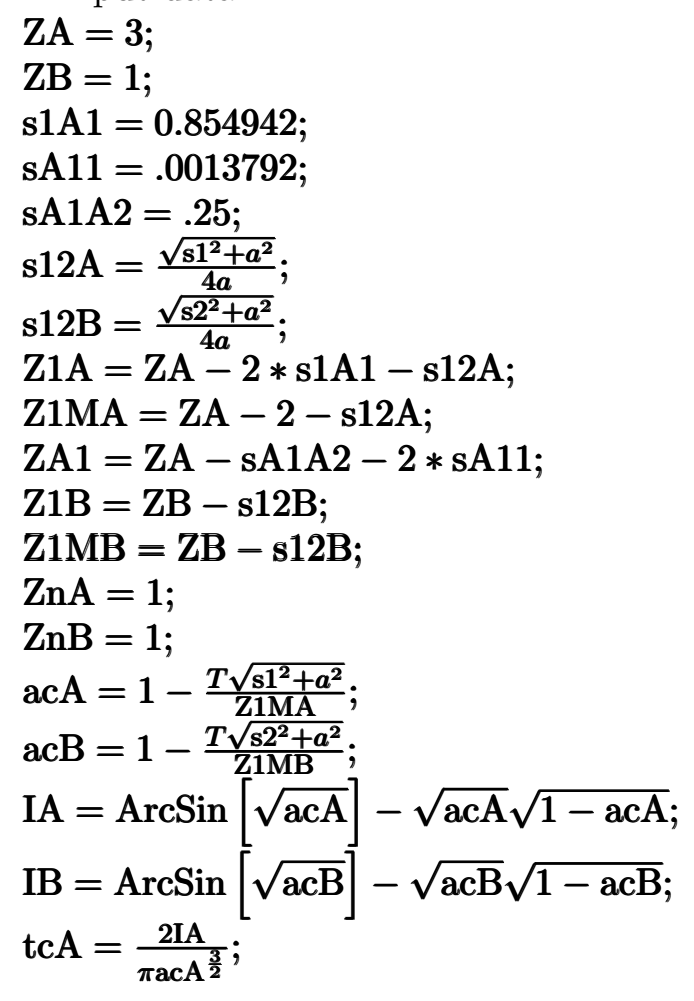




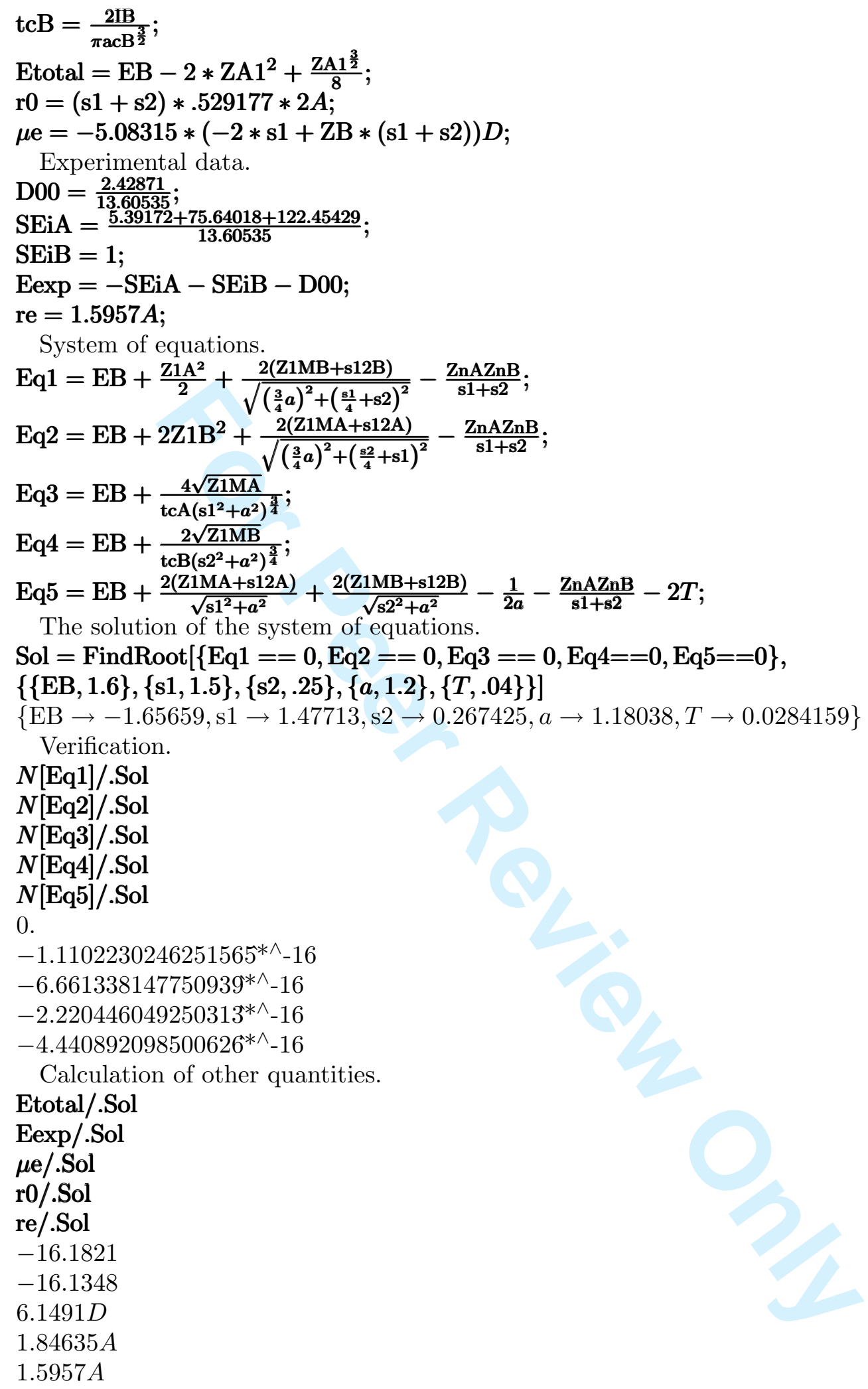

S3.7. Mathematica 7 script for the calculation of BeH.

The equivalences between the notations from paper and the symbols which are used in program are $Z_{A} \equiv Z A, Z_{B} \equiv Z B, \underline{T}_{m} \equiv T, s_{1, A 1} \equiv s 1 A 1, s_{A 1,1} \equiv s A 11$, $s_{A 1, A 2} \equiv s A 1 A 2, s_{1,2(A)} \equiv s 12 A, s_{1,2(B)} \equiv s 12 B, s_{1, A 3} \equiv s 1 A 3, Z_{1(A)} \equiv Z 1 A$, 
$Z_{1 M(A)} \equiv Z 1 M A, Z_{A 1} \equiv Z A 1, Z_{A 3} \equiv Z A 3, Z_{1(B)} \equiv Z 1 B, Z_{1 M(B)} \equiv Z 1 M B$, $\underline{\tilde{r}}_{A 3} \equiv r A 3, Z_{n A} \equiv Z n A, Z_{n B} \equiv Z n B, a_{c A} \equiv a c A, a_{c B} \equiv a c B, I_{A} \equiv I A, I_{B} \equiv I B$, $t_{c A} \equiv t c A, t_{c B} \equiv t c B, \underline{E} \equiv$ Etotal, $r_{0} \equiv r 0, \mu_{e} \equiv \mu e, \underline{D}_{0}^{0} \equiv D 00, \underline{S}_{E i A} \equiv S E i A$, $\underline{S}_{E i B} \equiv S E i B, \underline{E}_{e x p} \equiv \operatorname{Eexp}, r_{e} \equiv r e, \underline{E}_{b 1} \equiv E B 1, \underline{E}_{b 2} \equiv E B 2, \underline{a} \equiv a, \underline{\sigma}_{1} \equiv s 1$, $\underline{\sigma}_{2} \equiv s 2$.

The output data, given at the end of the script are, respectively, $\underline{E}, \underline{E_{e x p}}, \mu_{e}, r_{0}$ and $r_{e}$.

Input data.

$\mathrm{ZA}=4$

$\mathrm{ZB}=1$

$T=.015$

$\mathrm{s} 1 \mathrm{~A} 1=0.83882$

$\mathrm{sA11}=.00221203$

$\mathrm{sA} 1 \mathrm{~A} 2=.25$

$\mathrm{s} 12 \mathrm{~A}=\frac{\sqrt{\mathrm{s} 1^{2}+a^{2}}}{4 a}$

$\mathrm{s} 12 \mathrm{~B}=\frac{\sqrt{\mathrm{s} 2^{2}+a^{2}}}{4 a}$

$\mathrm{s} 1 \mathrm{~A} 3=\frac{\sqrt{2}}{4 * \sqrt{1+\frac{\mathrm{s} 1}{\sqrt{\mathrm{s}^{2}+a^{2}}}}}$

$\mathrm{Z} 1 \mathrm{~A}=\mathrm{ZA}-2 * \mathrm{~s} 1 \mathrm{~A} 1-\mathrm{s} 12 \mathrm{~A}-\mathrm{s} 1 \mathrm{~A} 3$

$\mathrm{Z} 1 \mathrm{MA}=\mathrm{ZA}-2-\mathrm{s} 12 \mathrm{~A}-\mathrm{s} 1 \mathrm{~A} 3$

$\mathrm{ZA} 1=\mathrm{ZA}-\mathrm{sA} 1 \mathrm{~A} 2-3 * \mathrm{sA} 11$

$\mathrm{ZA} 3=\mathrm{ZA}-2 * \mathrm{~s} 1 \mathrm{~A} 1-2 * \mathrm{~s} 1 \mathrm{~A} 3$

$\mathrm{Z} 1 \mathrm{~B}=\mathrm{ZB}-\mathrm{s} 12 \mathrm{~B}$

$\mathrm{Z} 1 \mathrm{MB}=\mathrm{ZB}-\mathrm{s} 12 \mathrm{~B}$;

$\mathrm{rA3}=\frac{3}{\mathrm{ZA}-2 * \mathrm{~s} 1 \mathrm{~A} 1}$

$\mathrm{ZnA}=\mathrm{ZA}-2-\frac{(\mathrm{s} 1+\mathrm{s} 2)^{2}}{(\mathrm{~s} 1+\mathrm{s} 2+\mathrm{rA} 3)^{2}}$

$\mathrm{ZnB}=1$

$\mathrm{acA}=1-\frac{T \sqrt{\mathrm{s}^{2}+a^{2}}}{\mathrm{Z1MA}}$

$\mathrm{acB}=1-\frac{T \sqrt{\mathrm{s}^{2}+a^{2}}}{\mathrm{Z1MB}}$

$\mathrm{IA}=\operatorname{ArcSin}[\sqrt{\mathrm{acA}}]-\sqrt{\mathrm{acA}} \sqrt{1-\mathrm{acA}}$

$\mathrm{IB}=\operatorname{ArcSin}[\sqrt{\mathrm{acB}}]-\sqrt{\mathrm{acB}} \sqrt{1-\mathrm{acB}}$

$\mathrm{tcA}=\frac{2 \mathrm{IA}}{\pi \mathrm{acA}^{\frac{3}{2}}}$

$\mathrm{tcB}=\frac{2 \mathrm{IB}}{\pi \mathrm{acB}^{\frac{3}{2}}}$

Etotal $=-\frac{\mathrm{ZA} 3^{2}}{4}-2 * \mathrm{ZA1}^{2}-\frac{2 *(\mathrm{Z} 1 \mathrm{MA}+\mathrm{s} 12 \mathrm{~A})}{\sqrt{\mathrm{s}^{2}+a^{2}}}-\frac{2 *(\mathrm{Z} 1 \mathrm{MB}+\mathrm{s} 12 \mathrm{~B})}{\sqrt{\mathrm{s}^{2}+a^{2}}}+\frac{1}{2 * a}-$

$\frac{1}{\mathrm{rA3}+\mathrm{s} 1+\mathrm{s} 2}+\frac{(\mathrm{ZA}-2) * \mathrm{ZB}}{\mathrm{s} 1+\mathrm{s} 2}+\frac{\mathrm{ZA} 1 \frac{3}{2}}{8}+2 * T$

$\mathrm{r} 0=(\mathrm{s} 1+\mathrm{s} 2) * .529177 * 2 A ;$

$\mu \mathrm{e}=-5.08315 *(-2 * \mathrm{~s} 1+\mathrm{ZB} *(\mathrm{~s} 1+\mathrm{s} 2)+\mathrm{rA} 3) D ;$

Experimental data.

$\mathrm{D} 00=\frac{2.034}{13.60355}$

$\mathrm{SEiA}=\frac{9.3227+18.21116+153.89661+217.71865}{13.60535} ;$

$\mathrm{SEiB}=1$

$\mathrm{Eexp}=-\mathrm{SEiA}-\mathrm{SEiB}-\mathrm{D} 00$;

re $=1.3426 A$;

System of equations.

$\mathrm{Eq} 1=\mathrm{EB} 1+\frac{\mathrm{Z1A}^{2}}{2}+\frac{2(\mathrm{Z} 1 \mathrm{MB}+\mathrm{s} 12 \mathrm{~B})}{\sqrt{\left(\frac{3}{4} a\right)^{2}+\left(\frac{\mathrm{s} 1}{4}+\mathrm{s} 2\right)^{2}}}-\frac{\mathrm{ZnAZnB}}{\mathrm{s} 1+\mathrm{s} 2} ;$ 
$\mathrm{Eq} 2=\mathrm{EB} 2+2 \mathrm{Z} 1 \mathrm{~B}^{2}+\frac{2(\mathrm{Z} 1 \mathrm{MA}+\mathrm{s} 12 \mathrm{~A})}{\sqrt{\left(\frac{3}{4} a\right)^{2}+\left(\frac{\mathrm{s} 2}{4}+\mathrm{s} 1\right)^{2}}}-\frac{\mathrm{ZnAZnB}}{\mathrm{s} 1+\mathrm{s} 2}$

$\mathrm{Eq} 3=\mathrm{EB} 1+\frac{4 \sqrt{\mathrm{Z1MA}}}{\mathrm{tcA}\left(\mathrm{si}^{2}+a^{2}\right)^{\frac{3}{4}}}$

$\mathrm{Eq} 4=\mathrm{EB} 2+\frac{2 \sqrt{\mathrm{Z} 1 \mathrm{MB}}}{\mathrm{tcB}\left(\mathrm{s}^{2}+a^{2}\right)^{\frac{3}{4}}}$

$\mathrm{Eq} 5=0.5 *(\mathrm{~EB} 1+\mathrm{EB} 2)+\frac{2(\mathrm{Z} 1 \mathrm{MA}+\mathrm{s} 12 \mathrm{~A})}{\sqrt{\mathrm{s}^{2}+a^{2}}}+\frac{2(\mathrm{Z} 1 \mathrm{MB}+\mathrm{s} 12 \mathrm{~B})}{\sqrt{\mathrm{s}^{2}+a^{2}}}-\frac{1}{2 a}-\frac{\mathrm{ZnAZnB}}{\mathrm{s} 1+\mathrm{s} 2}-2 T ;$

The solution of the system of equations.

$\mathrm{Sol}=$ FindRoot $[\{\mathrm{Eq} 1==0, \mathrm{Eq} 2==0, \mathrm{Eq} 3==0, \mathrm{Eq} 4==0, \mathrm{Eq} 5==0\}$,

$\{\{\mathrm{EB} 1,-2.3\},\{\mathrm{EB} 2,-2\},\{\mathrm{s} 1,1.35\},\{\mathrm{s} 2, .01\},\{a, 1\}\}]$

$\{\mathrm{EB} 1 \rightarrow-2.51578, \mathrm{~EB} 2 \rightarrow-2.11007, \mathrm{~s} 1 \rightarrow 1.34462, \mathrm{~s} 2 \rightarrow 0.0149424, a \rightarrow$ $0.979054\}$

Verification.

$N[\mathrm{Eq1}] / . \mathrm{Sol}$

$N$ [Eq2]/.Sol

$N$ [Eq3]/.Sol

$N$ [Eq4]/.Sol

$N$ [Eq5]/.Sol

$1.1102230246251565^{* \wedge}-15$

$-2.220446049250313^{* \wedge}-16$

0 .

$1.3322676295501878^{* \wedge}-15$

0 .

Calculation of other quantities.

Etotal/.Sol

Eexp/.Sol

$\mu \mathrm{e} /$.Sol

r0/.Sol

re/.Sol

$-30.4238$

$-30.4872$

$0.192613 D$

$1.4389 A$

$1.3426 A$

\section{S3.8. Mathematica 7 script for the calculation of $\mathrm{BH}$.}

The equivalences between the notations from paper and the symbols which are used in program are $Z_{A} \equiv Z A, Z_{B} \equiv Z B, s_{1, A 1} \equiv s 1 A 1, s_{A 1,1} \equiv s A 11, s_{A 1, A 2} \equiv$ $s A 1 A 2, \alpha \equiv$ alpha $, s_{1, A 3 \equiv s 1 A 3}, s_{A 3, A 4} \equiv s A 3 A 4, Z_{1(A)} \equiv Z 1 A, Z_{1 M(A)} \equiv Z 1 M A$, $Z_{A 1} \equiv Z A 1, Z_{A 3} \equiv Z A 3, Z_{A 4} \equiv Z A 4, Z_{2(B)} \equiv Z 2 B, Z_{2 M(B)} \equiv Z 2 M B$, e $\equiv$ excen $\underline{r} \equiv r, \underline{d} \equiv d, \underline{b} \equiv b, Z_{n A} \equiv Z n A, Z_{n B} \equiv Z n B, \underline{E} \equiv$ Etotal, $\underline{E}_{b} \equiv E B, r_{0} \equiv r 0$, $\mu_{e} \equiv \mu e, \underline{D}_{0}^{0} \equiv D 00, \underline{S}_{E i A} \equiv S E i A, \underline{S}_{E i B} \equiv S E i \bar{B}, \underline{E}_{e x p} \equiv \operatorname{Eexp}, r_{e} \equiv r e, \underline{a} \equiv a$, $\underline{\sigma}_{1} \equiv s 1, \underline{\sigma}_{2} \equiv s 2$.

The output data, given at the end of the script are, respectively, $\underline{E}, \underline{E}_{e x p}, \mu_{e}, r_{0}$ and $r_{e}$.

Input data.

$\mathrm{ZA}=5$

$\mathrm{ZB}=1$

$\mathrm{s} 1 \mathrm{~A} 1=0.862983$;

$\mathrm{sA11}=.00246735$;

$\mathrm{sA} 1 \mathrm{~A} 2=.25$ 
alpha $=63$

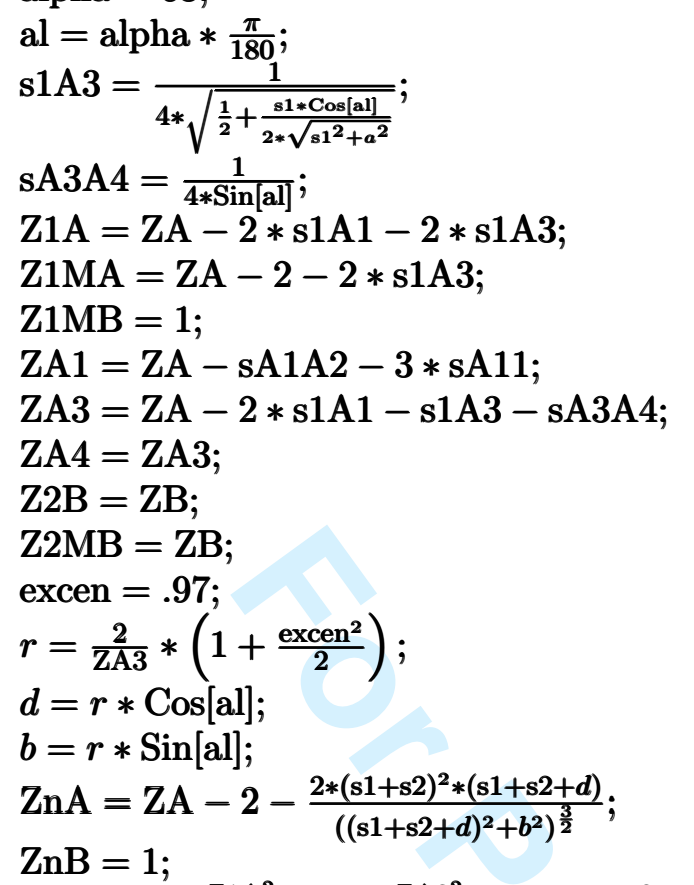

Etotal $=-\frac{{\mathrm{Z} 1 \mathrm{~A}^{2}}^{4}}{4}-1-\frac{\mathrm{ZA}^{2}}{2}-2 * \mathrm{ZA}^{2}+\frac{1}{\sqrt{\left(\frac{\mathrm{s} 1}{4}+\frac{\mathrm{s} 2}{4}\right)^{2}+\left(\frac{3}{2} * a\right)^{2}}}-\frac{1}{\sqrt{\left(\frac{s 1}{4}+\mathrm{s} 2\right)^{2}+\left(\frac{3}{4} a\right)^{2}}}-$ $\frac{\mathrm{ZA}-2}{\sqrt{\left(\frac{\mathrm{s} 2}{4}+\mathrm{s} 1\right)^{2}+\left(\frac{3}{4} a\right)^{2}}}+\frac{2}{\sqrt{\left(\frac{\mathrm{s} 2}{4}+\mathrm{s} 1+d\right)^{2}+\left(\frac{3}{4} a\right)^{2}+b^{2}}}-\frac{2}{\sqrt{(\mathrm{s} 1+\mathrm{s} 2+d)^{2}+b^{2}}}+\frac{\mathrm{ZA}-2}{\mathrm{~s} 1+\mathrm{s} 2}+\frac{\mathrm{ZA} 1 \frac{3}{2}}{8} ;$ $\mathrm{r} 0=(\mathrm{s} 1+\mathrm{s} 2) * .529177 * 2 A ;$

$\mu \mathrm{e}=-5.08315 *(-2 * \mathrm{~s} 1+\mathrm{ZB} *(\mathrm{~s} 1+\mathrm{s} 2)+2 * d) D$

Experimental data

$\mathrm{D} 00=\frac{3.42}{13.60535}$

$\mathrm{SEiA}=\frac{8.29803+25.15484+37.93064+259.37521+340.22580}{1360535}$

$\mathrm{SEiB}=1$;

$\mathrm{Eexp}=-\mathrm{SEiA}-\mathrm{SEiB}-\mathrm{D} 00$;

$\mathrm{re}=1.2324 A$

System of equations.

$\mathrm{Eq} 1=\mathrm{EB}+\frac{{\mathrm{Z} 1 \mathrm{~A}^{2}}^{4}}{4}{\mathrm{Z} 2 \mathrm{~B}^{2}}^{2}+\frac{\mathrm{Z1MB}}{\sqrt{\left(\frac{3}{4} a\right)^{2}+\left(\frac{\mathrm{s} 1}{4}+\mathrm{s} 2\right)^{2}}}+\frac{\mathrm{Z} 1 \mathrm{MA}}{\sqrt{\left(\frac{3}{4} a\right)^{2}+\left(\frac{\mathrm{s} 2}{4}+\mathrm{s} 1\right)^{2}}}-$

$\frac{1}{\sqrt{\left(2 * \frac{3}{4} a\right)^{2}+\left(\frac{s 1}{4}+\frac{s 2}{4}\right)^{2}}}-\frac{\mathrm{ZnAZnB}}{\mathrm{s} 1+\mathrm{s} 2}$

$\mathrm{Eq} 2=\mathrm{EB}+\frac{3 \sqrt{\mathrm{Z1MA}}}{\left(\mathrm{s}^{2}+a^{2}\right)^{\frac{3}{4}}}$

$\mathrm{Eq} 3=\mathrm{EB}+\frac{3 \sqrt{\mathrm{Z1MB}}}{\left(\mathrm{s}^{2}+a^{2}\right)^{\frac{3}{4}}}$

$\mathrm{Eq} 4=\mathrm{EB}+\frac{2 * \mathrm{Z} 1 \mathrm{MA}}{\sqrt{\mathrm{s}^{2}+a^{2}}}+\frac{2 * \mathrm{Z} 1 \mathrm{MB}}{\sqrt{\mathrm{s}^{2}+a^{2}}}-\frac{1}{2 a}-\frac{\mathrm{ZnAZnB}}{\mathrm{s} 1+\mathrm{s} 2}$

The solution of the system of equations.

$\mathrm{Sol}=$ FindRoot $[\{\mathrm{Eq} 1==0, \mathrm{Eq} 2==0, \mathrm{Eq} 3==0, \mathrm{Eq} 4==0\},\{\{\mathrm{EB}, 3.25\}$,

$\{\mathrm{s} 1, .876\},\{\mathrm{s} 2, .254\},\{a, .911\}\}]$

$\{\mathrm{EB} \rightarrow-3.19537, \mathrm{~s} 1 \rightarrow 0.888324, \mathrm{~s} 2 \rightarrow 0.261022, a \rightarrow 0.922599\}$

Verification.

$N[\mathrm{Eq} 1] /$. Sol

$N[\mathrm{Eq} 2] /$. Sol

$N[\mathrm{Eq} 3] /$. Sol

$N[\mathrm{Eq} 4] /$. Sol

$6.661338147750939^{* \wedge}-16$ 
$4.440892098500626^{* \wedge}-16$

$-8.881784197001252^{* \wedge}-16$

$4.440892098500626^{* \wedge}-16$

Calculation of other quantities.

Etotal/.Sol

Eexp

$\mu \mathrm{e} /$. Sol

r0/.Sol

re/.Sol

$-50.4587$

$-50.5691$

$-1.86638 D$

$1.21642 A$

$1.2324 A$

S3.9. Mathematica 7 script for the calculation of $\mathrm{CH}$.

The equivalences between the notations from paper and the symbols which are used in program are $Z_{A} \equiv Z A, Z_{B} \equiv Z B, s_{1, A 1} \equiv s 1 A 1, s_{A 1,1} \equiv s A 11, s_{A 1, A 2} \equiv s A 1 A 2$, $\alpha \equiv$ alpha $, s_{1, A 3 \equiv s 1 A 3}, s_{1, A 4 \equiv s 1 A 4}, s_{1, A 5 \equiv s 1 A 5}, s_{A 3, A 4} \equiv s A 3 A 4, Z_{1(A)} \equiv Z 1 A$, $Z_{1 M(A)} \equiv Z 1 M A, Z_{A 1} \equiv Z A 1, Z_{A 3} \equiv Z A 3, Z_{A 4} \equiv Z A 4, Z_{A 5} \equiv Z A 5, Z_{2(B)} \equiv$ $Z 2 B, Z_{2 M(B)} \equiv Z 2 M B, e \equiv$ excen $\underline{r} \equiv r, \underline{d} \equiv d, \underline{b} \equiv b, Z_{n A} \equiv Z n A, Z_{n B} \equiv Z n B$, $\underline{E} \equiv$ Etotal, $\underline{E}_{b} \equiv E B, r_{0} \equiv r 0, \mu_{e} \equiv \mu e, \underline{D}_{0}^{0} \equiv D 00, \underline{S}_{E i A} \equiv S E i A, \underline{S}_{E i B} \equiv S E i B$, $\underline{E}_{e x p} \equiv \operatorname{Eexp}, r_{e} \equiv r e, \underline{a} \equiv A, \underline{\sigma}_{1} \equiv s 1, \underline{\sigma}_{2} \equiv s 2$.

The output data, given at the end of the script are, respectively, $\underline{E}, \underline{E_{e x p}}, \mu_{e}, r_{0}$ and $r_{e}$.

Input data.

$\mathrm{ZA}=6$

$\mathrm{ZB}=1$

$\mathrm{s} 1 \mathrm{~A} 1=0.85505$

$\mathrm{sA} 11=.00269287$

$\mathrm{sA} 1 \mathrm{~A} 2=.25$

alpha $=67$

al $=$ alpha $* \frac{\pi}{180}$

$\mathrm{s} 1 \mathrm{~A} 3=\frac{1}{4 * \sqrt{\frac{1}{2}+\frac{s 1 * \cos [a \mathrm{al}]}{2 * \sqrt{s^{2}+a^{2}}}}}$

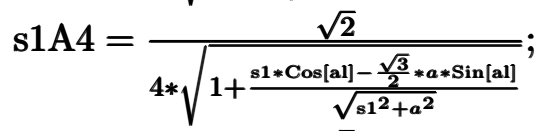

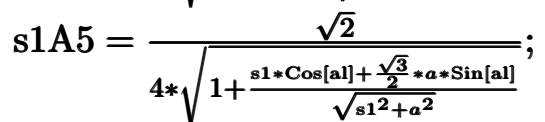

$\mathrm{sA3A} 4=\frac{1}{2 * \sqrt{3} * \operatorname{Sin}[\mathrm{al}]} ;$

$\mathrm{Z} 1 \mathrm{~A}=\mathrm{ZA}-2 \mathrm{~s} 1 \mathrm{~A} 1-\mathrm{s} 1 \mathrm{~A} 3-\mathrm{s} 1 \mathrm{~A} 4-\mathrm{s} 1 \mathrm{~A} 5$;

$\mathrm{Z} 1 \mathrm{MA}=\mathrm{ZA}-2-\mathrm{s} 1 \mathrm{~A} 3-\mathrm{s} 1 \mathrm{~A} 4-\mathrm{s} 1 \mathrm{~A} 5$

$\mathrm{Z} 1 \mathrm{MB}=1$

$\mathrm{ZA} 1=\mathrm{ZA}-\mathrm{sA} 1 \mathrm{~A} 2-4 * \mathrm{sA} 11$

$\mathrm{ZA} 3=\mathrm{ZA}-2 * \mathrm{~s} 1 \mathrm{~A} 1-2 * \mathrm{sA} 3 \mathrm{~A} 4-\mathrm{s} 1 \mathrm{~A} 3$

$\mathrm{ZA} 4=\mathrm{ZA}-2 * \mathrm{~s} 1 \mathrm{~A} 1-2 * \mathrm{sA} 3 \mathrm{~A} 4-\mathrm{s} 1 \mathrm{~A} 4$

$\mathrm{ZA} 5=\mathrm{ZA}-2 * \mathrm{~s} 1 \mathrm{~A} 1-2 * \mathrm{sA} 3 \mathrm{~A} 4-\mathrm{s} 1 \mathrm{~A} 5$

$\mathrm{Z} 2 \mathrm{~B}=\mathrm{ZB}$ 
$\mathrm{Z} 2 \mathrm{MB}=\mathrm{ZB}$

excen $=.98$

$r=\left(\frac{1}{\mathrm{ZA} 3}+\frac{1}{\mathrm{ZA} 4}+\frac{1}{\mathrm{ZA} 5}\right) * \frac{2}{3} *\left(1+\frac{\text { excen }^{2}}{2}\right)$

$d=r * \operatorname{Cos}[\mathrm{al}]$

$b=r * \operatorname{Sin}[\mathrm{al}]$

$\mathrm{ZnA}=\mathrm{ZA}-2-\frac{3 *(\mathrm{~s} 1+\mathrm{s} 2)^{2} *(\mathrm{~s} 1+\mathrm{s} 2+d)}{\left((\mathrm{s} 1+\mathrm{s} 2+d)^{2}+b^{2}\right)^{\frac{3}{2}}}$

$\mathrm{ZnB}=1$

Etotal $=-\frac{{\mathrm{Z} 1 A^{2}}^{4}}{4}-\frac{\mathrm{ZA}^{2}}{4}-\frac{\mathrm{ZA}^{2}}{4}-\frac{\mathrm{ZA}^{2}}{4}-1-2 * \mathrm{ZA}^{2}+\frac{1}{\sqrt{\left(\frac{\mathrm{si}}{4}+\frac{\mathrm{s2}}{4}\right)^{2}+\left(\frac{3}{2} * a\right)^{2}}}-$

$\frac{1}{\sqrt{\left(\frac{\mathrm{s} 1}{4}+\mathrm{s} 2\right)^{2}+\left(\frac{3}{4} a\right)^{2}}}-\frac{\mathrm{ZA}-2}{\sqrt{\left(\frac{\mathrm{s} 2}{4}+\mathrm{s} 1\right)^{2}+\left(\frac{3}{4} a\right)^{2}}}+\frac{1}{\sqrt{\left(\frac{3}{4} * a\right)^{2}+b^{2}+\left(\frac{\mathrm{s} 2}{4}+\mathrm{s} 1+d\right)^{2}}}+$

$\frac{1}{\sqrt{\left(\frac{3}{4} * a-\frac{\sqrt{3}}{2} * b\right)^{2}+\left(\frac{b}{2}\right)^{2}+\left(\frac{\mathrm{s} 2}{4}+\mathrm{s} 1+d\right)^{2}}}+\frac{1}{\sqrt{\left(\frac{3}{4} * a+\frac{\sqrt{3}}{2} * b\right)^{2}+\left(\frac{b}{2}\right)^{2}+\left(\frac{s 2}{4}+\mathrm{s} 1+d\right)^{2}}}-$

$\frac{3}{\sqrt{b^{2}+(\mathrm{s} 1+\mathrm{s} 2+d)^{2}}}+\frac{(\mathrm{ZA}-2) * \mathrm{ZB}}{\mathrm{s} 1+\mathrm{s} 2}+\frac{\mathrm{ZA} 1^{\frac{3}{2}}}{8}$

$\mathrm{r} 0=(\mathrm{s} 1+\mathrm{s} 2) * .529177 * 2 A$;

$\mu \mathrm{e}=-5.08315 *(-2 * \mathrm{~s} 1+\mathrm{ZB} *(\mathrm{~s} 1+\mathrm{s} 2)+3 * d) D ;$

Experimental data.

$\mathrm{D} 00=\frac{3.465}{13.60535}$

$\mathrm{SEiA}=\frac{11.26030+24.38332+47.8878+64.4939+392.087+489.99334}{13.60555} ;$

$\mathrm{SEiB}=1$;

$\operatorname{Eexp}=-\mathrm{SEiA}-\mathrm{SEiB}-\mathrm{D} 00$;

re $=1.1199 A$

System of equations.

$\mathrm{Eq} 1=\mathrm{EB}+\frac{\mathrm{Z1A}^{2}}{4}+\mathrm{Z2B}^{2}+\frac{\mathrm{Z1MB}}{\sqrt{\left(\frac{3}{4} a\right)^{2}+\left(\frac{\mathrm{s} 1}{4}+\mathrm{s} 2\right)^{2}}}+\frac{\mathrm{Z1MA}}{\sqrt{\left(\frac{3}{4} a\right)^{2}+\left(\frac{\mathrm{s} 2}{4}+\mathrm{s} 1\right)^{2}}}-$

$\frac{1}{\sqrt{\left(2 * \frac{3}{4} a\right)^{2}+\left(\frac{s 1}{4}+\frac{s 2}{4}\right)^{2}}}-\frac{\mathrm{ZnAZnB}}{\mathrm{s} 1+\mathrm{s} 2}$

$\mathrm{Eq} 2=\mathrm{EB}+\frac{3 \sqrt{\mathrm{Z1MA}}}{\left(\mathrm{si}^{2}+a^{2}\right)^{\frac{3}{4}}}$

$\mathrm{Eq} 3=\mathrm{EB}+\frac{3 \sqrt{\mathrm{Z2MB}}}{\left(\mathrm{s}^{2}+a^{2}\right)^{\frac{3}{4}}}$

$\mathrm{Eq} 4=\mathrm{EB}+\frac{2 * \mathrm{Z} 1 \mathrm{MA}}{\sqrt{\mathrm{s}^{2}+a^{2}}}+\frac{2 * \mathrm{Z} 1 \mathrm{MB}}{\sqrt{\mathrm{s}^{2}+a^{2}}}-\frac{1}{2 a}-\frac{\mathrm{ZnAZnB}}{\mathrm{s} 1+\mathrm{s} 2}$

Solutia sistemului de ecuatii

Sol $=$ FindRoot $[\{\mathrm{Eq} 1==0, \mathrm{Eq} 2==0, \mathrm{Eq} 3==0, \mathrm{Eq} 4==0\}$,

$\{\{\mathrm{EB}, 4.3\},\{\mathrm{s} 1, .796\},\{\mathrm{s} 2, .190\},\{a, .808\}\}]$

$\{\mathrm{EB} \rightarrow-4.29182, \mathrm{~s} 1 \rightarrow 0.840475, \mathrm{~s} 2 \rightarrow 0.175936, a \rightarrow 0.767725\}$

Verification.

$N[\mathrm{Eq} 1] /$. Sol

$N[\mathrm{Eq} 2] /$. Sol

$N[\mathrm{Eq} 3] /$. Sol

$N[\mathrm{Eq} 4] /$. Sol

4.440892098500626*^-16

0 .

$-8.881784197001252^{* \wedge}-16$

$8.881784197001252^{* \wedge}-16$

Calculation of other quantities.

Etotal/.Sol

Eexp

$\mu \mathrm{e} / . \mathrm{Sol}$

r0/.Sol

re/.Sol

$-76.6349$ 
$-76.968$

$-1.91195 D$

$1.07572 A$

$1.1199 A$ 
Dear Professor Head-Gordon,

Please receive the revised form of my manuscript TMPH-2010-0364, entitled "Unconventional semiclassical method for calculating the energetic values of diatomic molecules."

Below is the cover letter containing the response to the referee comments and the changes I have made in the revised manuscript.

Thank you very much,

Alexandru Popa

Cover letter containing the response to the referee comments and the changes in the revised manuscript of manuscript TMPH-2010-0364, entitled "Unconventional semiclassical method for calculating the energetic values of diatomic molecules," author Alexandru Popa

I would like to thank the referee for the analysis of my paper and for the suggestions and issues raised, which are very useful in order to improve the clarity of my presentation.

Below is my response to the issues raised by the referee, together with the changes I have made in the revised manuscript.

a) On the comment which refers to the central field method.

Since the behavior of stationary systems is described by the Schrodinger equation, the method to calculate the systems' energetic values presented in this paper is based on the general wave properties, which are synthesized in our paper [26]. The main property of these systems is that the geometric elements of the wave described by the Schrodinger equation in the space $\mathrm{R}^{3 \mathrm{~N}}$ of the electron coordinates, namely the $\Sigma$ wave surfaces and their $\mathrm{C}$ normal curves, are solutions of the Hamilton-Jacobi equation written for the same system. The motion of the $\Sigma$ surfaces is periodical and the C curves are closed. A. Luis proved the same property in a completely different way [32], by showing that the discontinuities of the second derivatives of the wave function propagate on trajectories that result from the Hamilton-Jacobi equation, written for the same system. On the other hand, according to the theory of differential equations, these discontinuities occur across characteristic surfaces of the wave equation, which are the wave surfaces of the system. It results the perfect equivalence between the properties which have been proven in [26] and [32].

From here it follows the idea of our approach: instead of finding the wave function $\psi$, we use the properties of the curve $\mathrm{C}$, in order to calculate the energetic values, because $\psi$ and $\mathrm{C}$ belong to the same physical system. To accomplish this, we calculate exactly the projections of the $\mathrm{C}$ curve from the space $\mathrm{R}^{3 \mathrm{~N}}$ of the electron coordinates to the tridimensional spaces of the coordinates of the electrons of the systems, denoted by $\mathrm{C}_{\mathrm{a}}$ in the cases of simple systems. For complex systems, a very good approximation of the $\mathrm{C}_{\mathrm{a}}$ curves results using the central field approximation. More simply, we have to calculate or to approximate the classical periodic solutions of the system. 
The main mathematical problem, which is the calculation of the electrostatic energy between two electrons that move in the field of the same nucleus, is strongly simplified by the symmetry property of the system, which makes possible the calculation of the reciprocal screening coefficients between electrons. The expression of this electrostatic energy is given by relation (10). In this case, the motions of the electrons are separated, and each electron moves in the central field of the nucleus, having an effective order number, in the averaged field of the other electrons. These last two properties are the attributes of the central field method. At this point, the parallelism between the central field method in the case of the quantum calculation and in the case of semiclassical calculation is perfect.

This parallelism remains valid in the case of molecules because we proved that the $C_{1}$ curve corresponding to a molecular bond is composed of two atomic curves, which are denoted by $C_{1 A}$ and $C_{1 B}$. In this case, in virtue of relation (A3), the motion of the $e_{1}$ bond electron on the curve $\mathrm{C}_{1 \mathrm{~A}}$ is reduced to that in a central field, and $\mathrm{e}_{1}$ moves in the averaged field of the electron $\mathrm{e}_{2}$ and of the nucleus $\mathrm{n}_{\mathrm{B}}$. Similar properties are valid when the electron moves on the $\mathrm{C}_{1 \mathrm{~B}}$ curve.

Revision: In Section 2, I introduced the following paragraph after Eq. (11):

"The model represented by equations (3)-(10) has two properties which are both attributes of a central field type solution. The first is that the motion of an arbitrary electron is similar to that in a hydrogenoid system, and it takes place in the averaged field of the other electrons. The effect of the interaction with these electrons is included in the effective order number of the nucleus. The second property, which is useful in applications, is that adding a supplementary electron to the system leads only to the modification of the effective order numbers corresponding to the other electrons, while their equations remain unchanged."

A second revision, which refers to the central field method applied in the case of the calculation of the $\mathrm{C}_{\mathrm{a}}$ curves of the bond electrons is made in the paragraph which has been introduced at the end of the Section 3, which refers also to the modeling of the lone pair electrons. See the revisions made at the point $d$ ).

b) On the comment which refers to the calculation of the bond length.

Our calculations take into account all the components of the total energy of the system, with one exception, which results from the hypothesis (h5), namely kinetic energies of the nuclei are zero. On the other hand, the potential energy of the interaction between nuclei is taken into account in our calculations. It follows that the distance between nuclei, which is denoted by $r_{0}$ in the manuscript, corresponds to values of the nuclei velocities of zero. The experimental value of the distance between nuclei, which is denoted by $r_{e}$ in [34], corresponds to the minimum of the Morse curve, which stands for the maximum velocities of the nuclei. This explains the differences between the values of $r_{0}$ and $r_{e}$ : the former corresponds to zero velocities of the nuclei, while the latter corresponds to maximum velocities of the nuclei. 


\section{Revisions: \\ - $\quad$ In the revised manuscript we introduced Table 3 which contains a comparison between the values of $r_{0}$ and $r_{e}$, which is taken from [29]. The table contains also the domains of the values of the distances between nuclei, calculated by the Hartree-Fock method, which are taken from [29] as well. \\ - The paragraphs before and after Eq. (44) in Section 4 are changed as follows:}

"In virtue of hypothesis (h5), the kinetic energy of the nuclei is zero. It follows that the distance between nuclei, denoted by $\mathrm{r}_{0}$ and calculated with the relation

corresponds to values of the nuclei velocities of zero. On the other hand, the experimental value of the distance between nuclei, denoted by $r_{e}$, corresponds to the minimum of the Morse curve [34]. The theory of the harmonic oscillation of the nuclei shows that this minimum corresponds to maximum velocities of the nuclei. It follows that $r_{0}$ is different from $r_{e}$ because the first corresponds to zero velocities of the nuclei, while the last corresponds to the maximum velocities of the nuclei."

- In Section 5, I changed the paragraph following Eq. (46). It now reads:

"The values of $\mathrm{r}_{0}$, calculated from (44), and the experimental value of the distance between nuclei, denoted by $r_{e}$ and taken from Ref. [29], are given in angstroms in Table 3 . Table 3 also gives the range of values for the distances between nuclei, denoted by $\mathrm{r}_{\mathrm{eHF}}$, calculated with the Hartree-Fock method and taken from [29]."

c) On the comment which refers to the shape of the curve $\mathrm{C}_{1 \mathrm{~A}}$ from Fig. 1 .

The $\mathrm{C}_{\mathrm{a}}$ curves for $\mathrm{s}$ states and those resulting from the exact solutions in the cases of boron or carbon atoms [28] are elliptic quasilinear curves (sometimes called pendular curves). These ellipses have $b>>a$, where $b$ and a are the ellipse semiaxes. Consequently, the upper and lower parts of each ellipse are very close, and, for simplicity the ellipse is drawn as a straight line. This is why the curve $\mathrm{C}_{1 \mathrm{a}}$ in Fig. 1 is drawn as a straight line in the vicinity of the nucleus $n_{a}$. However, we have to imagine it as a very thin ellipse which surrounds the nucleus.

Revision: The second paragraph after Eqs. (16) and (17), in Section 3, is modified to read as follows

"......... For this reason, in this paper we restrict ourselves to the case in which the molecular $\mathrm{C}_{1}$ curve is composed of two atomic curves, denoted by $\mathrm{C}_{1 \mathrm{~A}}$ and $\mathrm{C}_{1 \mathrm{~B}}$ which are ellipses with eccentricities very close to unity, as shown in Fig. 1. At the scale of the figure, these ellipses are drawn as straight lines in the vicinities of the nuclei, in spite of 
the fact that the curve $C_{1 A}$ surrounds the nucleus $n_{A}$ while the curve $C_{1 B}$ surrounds the nucleus $\mathrm{n}_{\mathrm{B}}$. The curves $\mathrm{C}_{1}$ and $\mathrm{C}_{2}$ depend on three parameters: ......."

d) On the comment which refers to the lone pair electrons.

The interaction between the electrons that move in the field of the same nucleus, in the case of the central field approach, is reflected by the reciprocal screening coefficient that enters in the expression of the effective order number of the nucleus. A general property results: the addition of a new electron to the system modifies only the expression of the effective order number of the nucleus, but leaves unchanged the equations of motion of the electrons.

In view of this property, the calculation of the Ca curves of the bond electrons is done in an identical manner regardless of whether there are valence electrons that do not participate in the bond or there are no such valence electrons. More specifically, in Section 3.1 we analyzed the case of no non-bonding electrons as a general recipe that allows the calculation of Ca curves. Subsequently, we showed that, adding a non-bonding valence electron does not change the system of equations that leads to the curve $C_{a}$. Instead the only modification is in the expression of the effective order number.

Revision: At the end of the Section 3 we have introduced the following paragraph in order to explain these points better:

"In this section we have calculated the $C_{a}$ curves when the bond electrons move in the fields of two nuclei and the system contains only valence electrons which participate to the bond. In Appendix we proved that the motion of a bond electron in the case of homonuclear molecules with simple bond is similar to that in a central field. An example of such an electron is $\mathrm{e}_{1}$ which moves on curves $\mathrm{C}_{1 \mathrm{~A}}$ and $\mathrm{C}_{1 \mathrm{~B}}$. This property results from relation (A3). Identical properties are proved in the Supplementary Online Material for other types of bonds. It follows from Section 2 that, in virtue of the second property of motion in the central field, adding a supplementary electron that does not participate to the bond in the field of the nucleus $n_{A}$ does not change the equation of motion of the electron $\mathrm{e}_{1}$. The only change is the fact that the expression of $\mathrm{Z}_{1}$ contains a supplementary screening coefficient corresponding to the interaction between $\mathrm{e}_{1}$ and the supplementary electron. In the next section we will see that for the general case of the molecules which contains electrons which do not participate to the bond, the relations from Appendix remain valid, with the only difference that the expressions of the order numbers are changed."

e) On the comment referring to the sigma and pi nature of the double bond.

The parallelism between the central field approaches in quantum and classical calculations goes far in the cases of the hybrid solutions which appear when the number of the electrons is increased. The hybrid solutions are imposed by the symmetry properties of the system. For example, in the case of the carbon atom, the wave function 
of the valence electrons is invariant under the symmetry operations of the regular tetrahedron (RTH) configuration [33], and leads to symmetrical orbitals, having identical shapes. We have shown that this property results rigorously from the classical equations [28], because the $C_{a}$ curves have a tetrahedron configuration in the case of the carbon atom. These curves are hybrid ones, of sp type, because they have identical shapes and their angular momentum is between a negligible value and $\sqrt{2} \bar{h}$, which correspond, respectively, to the 2 s and 2 p curves [28].

As we have shown in Section 3, the molecular curves corresponding to the simple, double and triple bonds are combinations of the sp hybrid atomic curves. As a consequence, the $\mathrm{C}_{\mathrm{a}}$ curves of the double bond have a sigma and pi nature, because they are combinations of $\mathrm{sp}$ atomic curves. The hybrid character of these curves is reflected, as in the case of the atomic curves, by the fact that they are symmetrical and their shapes are identical.

f) On the comment which refers to the references.

Answer and revisions: In response to the refree's comment I introduced 16 new references. In addition I have modified the first paragraph of the Introduction, as follows:

"Over the last few years, a large number of semiclassical approaches were published in the field of atomic and molecular systems [1]-[5]. The semiclassical methods lead to solutions which are relatively easy to calculate, using the information obtained from the study of classical trajectories. In numerous papers the semiclassical eigenvalues of the bond states are related to the periodic trajectories [6]-[12], in others they are related to the phase manifolds generated by quasiperiodic trajectories [13]-[15]. In this family of topics we can consider also the study of the semiclassical trajectories with the aid of the propagator functions [16]-[20] or the application of a generalized semiclassical theory to multidimensional dynamical systems [21]. In this paper we present a multidimensional semiclassical analysis which applies to a wide class of atoms and molecules."

Thank you very much, Alexandru Popa 


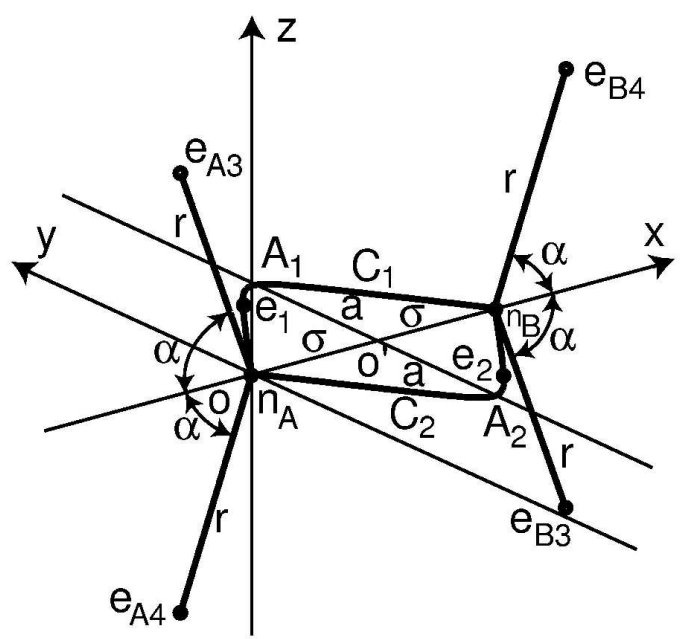

$148 \times 123 \mathrm{~mm}(600 \times 600 \mathrm{DPI})$ 


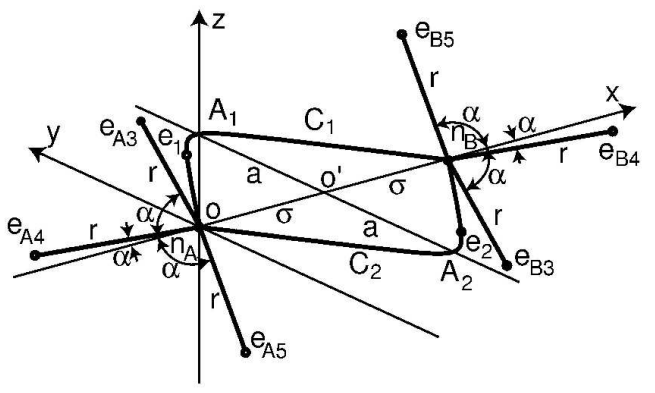

(a)

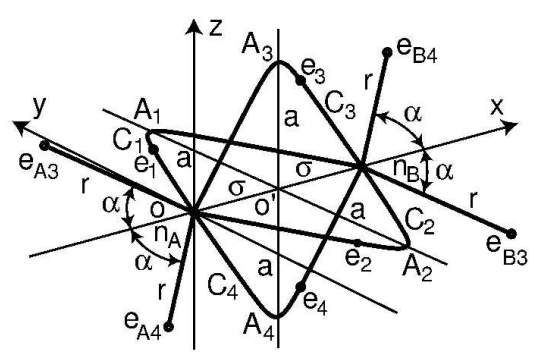

(b)

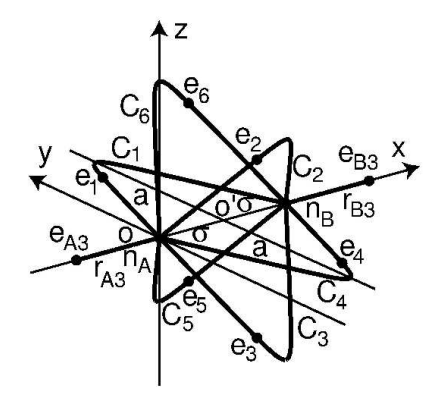

(c)

$154 \times 261 \mathrm{~mm}(600 \times 600 \mathrm{DPI})$

URL: http://mc.manuscriptcentral.com/tandf/tmph 


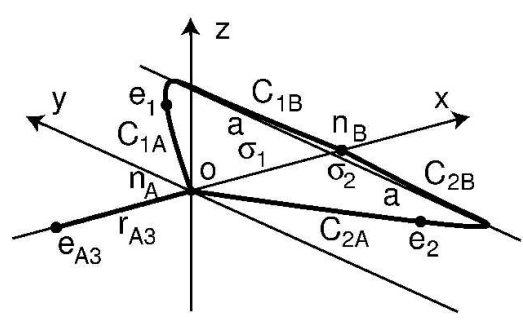

(a)

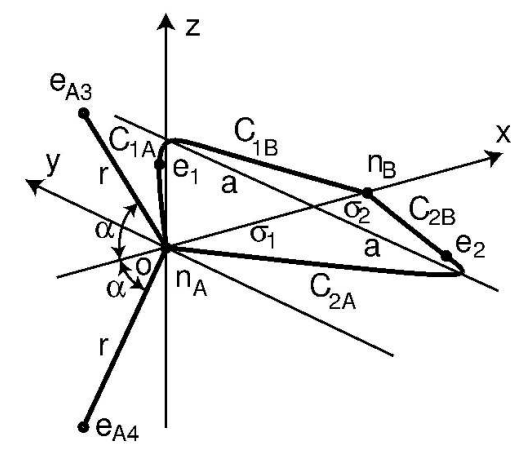

(b)

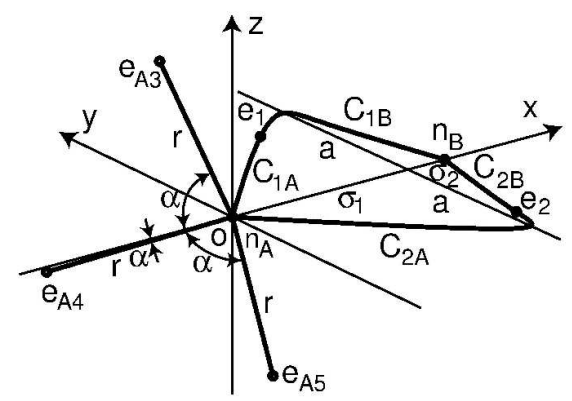

(c)

$192 \times 242 \mathrm{~mm}(600 \times 600 \mathrm{DPI})$ 